SLAC-TN-07-006

LBNL- 60958

ST/F-TN-06/15

July 6, 2006

\title{
FERMI\&Elettra Accelerator Technical Optimization Final Report
}

M. Cornacchia, P. Craievich, S. Di Mitri, Sincrotrone Trieste, Trieste, Italy

I. Pogorelov, J. Qiang, M. Venturini, A. Zholents \#, LBNL, Berkeley, CA 94720, U.S.A.

D. Wang, MIT, Cambridge, MA 02139, U.S.A.,

R. Warnock, SLAC, Stanford, CA 94025, U.S.A 


\section{Content}

1 Introduction

1.1 Overview of design requirements

1.2 Underlining physics phenomena

2 Longitudinal beam dynamics

2.1 Longitudinal dynamics without collective effects.

2.2 Longitudinal dynamics with collective effects.

2.2.1 Acceleration sections

2.2.2 Longitudinal wake potentials

2.2.3 Coherent synchrotron radiation

2.2.4 Reverse tracking

2.2.5 Microbunching instability

2.2.5.1 Gain function

3 Machine design

2.2.5.2 Landau damping with the laser heater

3.1 Laser heater

3.2 First bunch compressor

3.2.1 Bifurcation in the phase space and means to control it

3.3 Second bunch compressor

3.4 End of the linac and the spreader

4. Transverse beam dynamics

4.1 Transverse dynamics without collective effects

4.1.1 Chromatic aberrations

4.1.1.1 Dipoles

4.1.1.2 Quadrupoles

4.1.2 Geometrical aberrations

4.1.2.1 Dipoles

4.1.3 Quantum fluctuations (ISR)

4.2 Trajectory distorsion

4.2.1 Misalignment and field errors

4.2.1.1 Dipoles

4.2.1.2 Quadrupoles

4.2.2 Launching error

4.2.3 Trajectory correction

4.3 Transverse dynamics with collective effects

4.3.1 Transverse wake fields

4.3.1.1 Wake function for SOA and SOB structures

4.3.1.2 Wake function for $\mathrm{C}$ structures

4.3.1.3 Wake function for BTW structures

4.3.2 Beam break-up instability

4.3.2.1 Theoretical model

4.3.2.1.1 Effect of an initial lateral displacement

4.3.2.1.2 Effect of misaligned BTW accelerating structures

4.3.2.2 Control of the BBU induced banana shape

4.3.2.2.1 Effect of trajectory jitter on the banana shape 
4.4 Transversal acceptance and error tolerances

4.4.1 Beam size and aperture

4.4.2 Misalignment

4.4.3 Field quality

4.4.4 Optical mismatch at the injection

5 Tracking studies without errors

5.1 Short bunch case

5.2 Medium bunch case

5.3 Long bunch case

6. Effect of errors

6.1 Effect of errors on longitudinal dynamics

6.1.1 Simulation technique

6.1.2 Jitter sensitivities and tolerance budgets

6.1.2.1 Medium bunch cases

6.1.2.2 Long bunch cases

6.1.3 Global jitter studies

6.1.3.1 Medium bunch cases

6.1.3.2 Long bunch cases

6.1.4 Slice jitter analyses

6.2 Tracking studies with errors

Appendix A: 6D parallel tracking code IMPACT

Appendix B: Method of Vlasov's equation

References 


\section{Introduction}

This chapter describes the accelerator physics aspects, the engineering considerations and the choice of parameters that led to the accelerator design of the FERMI Free-Electron-Laser. The accelerator (also called the "electron beam delivery system") covers the region from the exit of the injector to the entrance of the first FEL undulator. The considerations that led to the proposed configuration were made on the basis of a study that explored various options and performance limits. This work follows previous studies of X-ray FEL facilities (SLAC LCLS [1], DESY XFEL [2], PAL XFEL [3], MIT [4], BESSY FEL[5], LBNL LUX [6], Daresbury 4GLS [7]) and integrates many of the ideas that were developed there. Several issues specific to harmonic cascade FELs, and that had not yet been comprehensively studied, were also encountered and tackled. A particularly difficult issue was the need to meet the requirement for high peak current and small slice energy spread, as the specification for the ratio of these two parameters (that defines the peak brightness of the electron beam) is almost a factor of two higher than that of the LCLS's SASE FEL. Another challenging aspect was the demand to produce an electron beam with as uniform as possible peak current and energy distributions along the bunch, a condition that was met by introducing novel beam dynamics techniques. Part of the challenge was due to the fact that there were no readily available computational tools to carry out reliable calculations, and these had to be developed. Most of the information reported in this study is available in the form of scientific publications, and is partly reproduced here for the convenience of the reader.

\subsection{Overview of design requirements}

FERMI accelerator is schematically shown in Figure (1.1). It consists of four linacs, two bunch compressors, a laser heater, and a spreader. The latter directs the electron beam into one of two undulator lines (not shown).

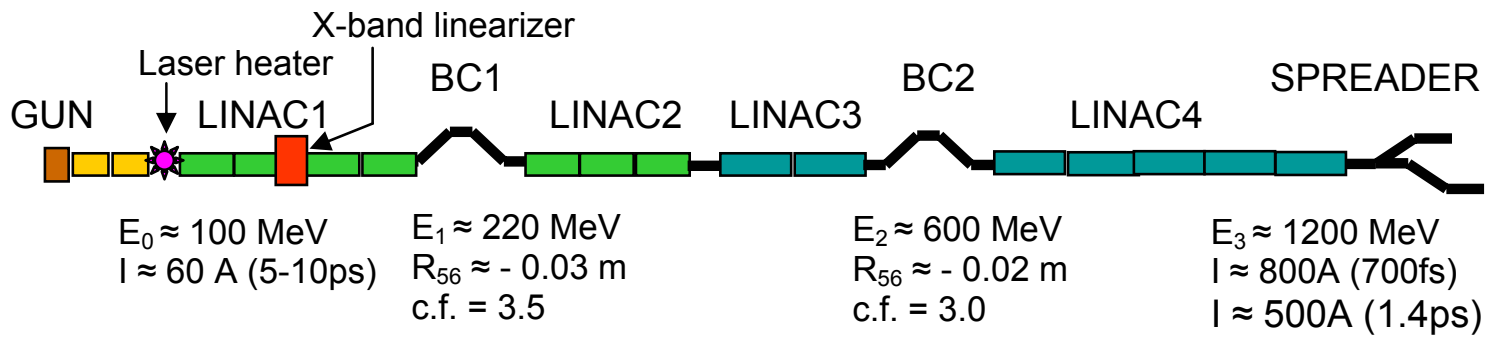

Figure (1.1) A schematic of the FERMI accelerator.

The laser heater defines the end of the injector and the start of the accelerator. At this point the energy of the electron beam is $\sim 100 \mathrm{MeV}$ and the peak current is $60-70 \mathrm{~A}$. At the end of acceleration the electron beam energy is approximately $1.2 \mathrm{GeV}$ and the electron peak current is $500 \mathrm{~A}$ or $800 \mathrm{~A}$, depending on the bunch length needed by the 
FEL processes. The latter envisage three options for the electron bunch length: the short bunch option with a bunch length of the order of $200 \mathrm{fs}$, the medium bunch option with a bunch length of the order of $700 \mathrm{fs}$ and the long bunch option with a bunch length of the order of $1.4 \mathrm{ps}$. The electron beam delivery system was designed with sufficient flexibility to accommodate these options. Other important electron beam parameters include the normalized slice electron beam emittance and the slice energy spread, which are 1.5 micron and $150 \mathrm{keV}$ respectively for all three bunch lengths. The list of the design parameters is summarized in Table (1.1). A new parameter the "flatness" is also quoted. It defines that value of the quadratic component of the average energy variation along the bunch for which the increase in bandwidth of the x-ray signal due to this variation becomes equal to the Fourier transform limited bandwidth defined by the bunch length.

\section{Table (1.1) Main electron beam parameters}

$\begin{array}{lccc} & \text { Short } & \text { Medium } & \text { Long } \\ \text { Bunch length, fs (flat part) } & 200 & 700 & 1400 \\ \text { Peak current, A } & 800 & 800 & 500 \\ \text { Emittance(slice), } \mu \mathrm{m} & <1.5 & <1.5 & <1.5 \\ \text { Energy spread(slice), keV } & <150 & <150 & <150 \\ \text { Flatness, }\left|\mathrm{d}^{2} \mathrm{E} / \mathrm{dt}^{2}\right|, \mathrm{MeV}^{2} \mathrm{ps}^{2} & - & <0.8 & <0.2\end{array}$

Table 1 indicates that the operation of the FERMI FEL requires high peak current, low emittance and low energy spread. The injector provides the low emittance and energy spread, while the accelerator must provide the high peak current. Preservation of the beam quality during bunch compression and acceleration is the most challenging task of the accelerator optimization study.

\subsection{Underlining physics phenomena}

When the intense electron bunch propagates down the accelerator, it is subject to various collective effects. Among them are the space charge effects and it appears that the longitudinal space charge effects (LSC) are more important than transverse as they continue to play a visible role in the formation of the microstructures on the electron bunch even at high energies. Longitudinal and transverse wake-fields are also extremely important. In particular, in the FERMI accelerator, the longitudinal wake- fields are so strong that they must be accounted for in the process of setting up the energy chirp for bunch compression. It was found that the longitudinal wake- fields are also responsible for the nonlinear variation of the medium slice energy along the bunch.

Transverse wake fields are also important since, if uncorrected, they can damage the electron beam quality. Also relevant is the emission of coherent synchrotron radiation (CSR) in the bunch compressors and the spreader. For perfectly smooth bunch without any internal microstructures, the effect of the CSR is similar to the effect of the longitudinal wake-fields, but with the additional threat of emittance dilution. In presence of a microbunching structure in the beam, the CSR tends to enhance it, thus giving rise to 
the so-called microbunching instability. In general, the microbunching instability is driven by a combination of LSC, bunch compression and CSR and, if unaccounted for, can increase the energy spread in the electron beam beyond the tolerance for proper FEL operation.

Other sources of possibly harmful effects are the nonlinear components in the rf waveform and the nonlinear components in the time-of-flight characteristics of the bunch compressor. Together, they are often responsible for appearance of the high spikes in the peak current at the edges of the electron bunch. Such spike at the head of the bunch can induce resistive wall wake-fields that will energy modulate the electron bunch during its motion through the spreader and_undulator. A spike at the tail of the bunch can induce energy variation along the bunch due to the CSR in the spreader.

All of the issues listed above are addressed in this chapter.

\section{Longitudinal beam dynamics}

One of the project goals is to produce an electron beam with high peak current and small energy spread. In order achieve this aim, the electron beam must be manipulated in longitudinal phase space. This is normally achieved by using a series of rf accelerating sections and magnetic chicanes (henceforth called the "bunch compression system"). It is desirable that this process remains linear in order to avoid bifurcation in the longitudinal phase space and high peak current spikes at the edges of the electron bunch. A distribution as uniform as possible is also efficient, as it provides the maximum peak current in the main body of the bunch for a given bunch length. Finding an optimum set of parameters is a rather complex and iterative process. Fortunately, for the most of the underlining physics phenomena the longitudinal dynamics is independent of the transverse dynamics and can be studied separately.

\subsection{Longitudinal dynamics without collective effects.}

Although collective effects play an important role in the formation of intense electron bunches, it is convenient first to ignore them and preview the dynamics of the bunch compression using analytical tools. Figure (2.1) shows a generic idea for bunch compression.

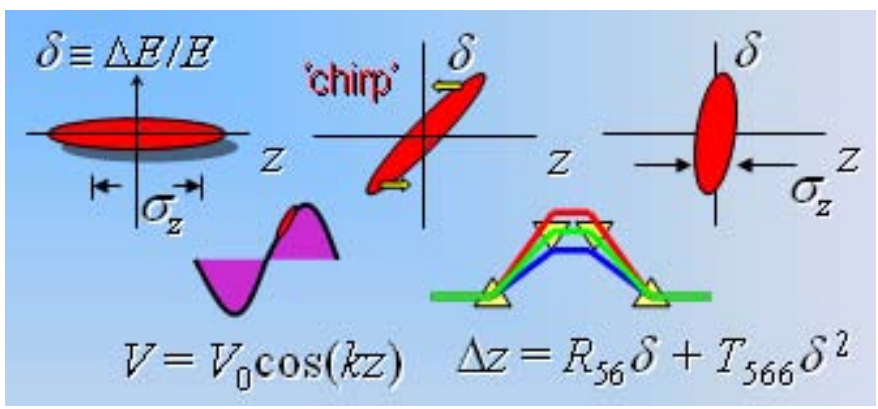

Figure (2.1) A schematic of the $r f$ bunch compression technique 
The rf system accelerates the electron beam off-crest and generates a correlated energy variation (chirp) along the electron bunch. The momentum compaction, the $R_{56}$ and $T_{566}$ terms in the transport matrix, relate the electron energy and the trajectory path length in the chicane in such a way that the higher energy electrons follow shorter paths and the lower energy electrons follow longer paths. This correlation between energy and path length compresses in length an electron bunch which is accelerated off-crest in the rf wave, as shown in Fig.(2.1) with the head at $\mathrm{z}<0$.

This scheme was implemented in the FERMI design. It was soon realized that another element, the 4-th harmonic cavity called the linearizer in Fig.(1.1), was also needed. This element helps linearize the chirp before $\mathrm{BC} 1[7,8]$. The acceleration seen by the electrons upstream of $\mathrm{BC} 1$ as a function of the distance of the electrons, $s$, from the center of the bunch can be written as:

$$
U(s)=U_{0} \cos (k s)+U_{1} \cos \left(k s+\phi_{1}\right)+U_{4} \cos \left(4 k s+\phi_{4}\right),
$$

where $k=2 \pi / \lambda_{r f}$ is the rf wave number and $\lambda_{r f}$ is the rf wavelength. $U_{0}$ is the acceleration amplitude of the two accelerating sections preceding the laser heater (see, Fig.(1.1)) where acceleration is on-crest. $U_{1}$ is the acceleration amplitude of the four accelerating sections placed between the laser heater and $\mathrm{BC} 1$, with off-crest acceleration at phase $\phi_{1}$ (referred to the crest of the rf wave). $U_{4}$ and $\phi_{4}$ are the amplitude and phase of the 4-th harmonic $\mathrm{x}$-band cavity. The first, second and third derivatives of $U(z)$ taken at the bunch center $s=0$ can be written:

$$
\begin{aligned}
& U_{(s=0)}^{\prime}=-k U_{1} \sin \left(\phi_{1}\right)-4 k U_{4} \sin \left(\phi_{4}\right) \\
& U_{(s=0)}^{\prime \prime}=-k^{2} U_{0}-k^{2} U_{1} \cos \left(\phi_{1}\right)-16 k^{2} U_{4} \cos \left(\phi_{4}\right) \\
& U_{(s=0)}^{\prime \prime \prime}=k^{3} U_{1} \sin \left(\phi_{1}\right)+64 k^{3} U_{4} \sin \left(\phi_{4}\right)
\end{aligned}
$$

The second derivative is zero if

$$
U_{4}=-\frac{U_{0}+U_{1} \cos \left(\phi_{1}\right)}{16 \cos \left(\phi_{4}\right)}
$$

thus eliminating a quadratic energy chirp. At the same time the first derivative becomes

$$
U_{(s=0)}^{\prime}=-k U_{1} \sin \left(\phi_{1}\right)+\frac{1}{4} k\left(U_{0}+U_{1} \cos \left(\phi_{1}\right)\right) \tan \left(\phi_{4}\right)
$$

A comparison of (2.2a) and (2.2b) shows that $U_{(z=0)}^{\prime \prime \prime}$ can also be written as:

$$
U_{(s=0)}^{\prime \prime \prime}=-k^{2} U_{(s=0)}^{\prime}+\frac{15}{4} k^{3}\left(U_{0}+U_{1} \cos \left(\phi_{1}\right)\right) \tan \left(\phi_{4}\right)
$$

This result will be used later. 
Defining $\Delta E\left(s_{i}\right) \equiv \frac{E\left(s_{i}\right)-E(0)}{E(0)}$, where $i$ indicates $i$-th electron at location $s_{i}$ with the relative energy $\delta_{i}$ before $\mathrm{BC} 1$, we obtain for its energy and coordinate after $\mathrm{BC} 1$ :

$$
\begin{aligned}
& \Delta E\left(s_{i}, \delta_{i}\right)=\left[-U_{1} \sin \left(\phi_{1}\right)+\frac{1}{4}\left(U_{0}+U_{1} \cos \left(\phi_{1}\right)\right) \tan \left(\phi_{4}\right)\right] \frac{k s_{i}}{U(0)}+\delta_{i}=h s_{i}+\delta_{i} \\
& s\left(s_{i}, \delta_{i}\right)=s_{i}+R_{56} \Delta E\left(s_{i}, \delta_{i}\right)=\left(1+h R_{56}\right) s_{i}+R_{56} \delta_{i}
\end{aligned}
$$

Notice a new notation $h$ in the right hand part of the first equation (2.6). Using (2.6) we can calculate the rms bunch length after the $\mathrm{BC} 1$ :

$$
\sigma_{z}=\left\langle s^{2}-\langle s\rangle^{2}\right\rangle=\sqrt{\left(1+h R_{56}\right)^{2} \sigma_{z 0}^{2}+\left(R_{56} \sigma_{\delta 0}\right)^{2}} \approx\left|1+h R_{56}\right| \sigma_{z 0},
$$

where $\sigma_{z 0}$ and $\sigma_{\delta 0}$ are the rms bunch length and uncorrelated energy spread before BC1. The compression factor is

$$
C=\frac{\sigma_{z 0}}{\sigma_{z}} \approx \frac{1}{1+h R_{56}}
$$

The uncorrelated energy spread after the compression becomes $C \sigma_{\delta 0}$.

Although, the analysis was carried out for $\mathrm{BC} 1$, similar considerations can be drawn for a second stage of bunch compression using linac2 and linac3 and $\mathrm{BC} 2$.

\subsection{Longitudinal dynamics with collective effects.}

\subsubsection{Acceleration sections}

The accelerator uses three type of sections. The S0A and S0B sections (yellow colored in Fig.(1.1)) follow the electron gun and are used for the initial acceleration and the emittance compensation scheme. Seven CERN-type sections (C1-C7, green colored in Fig.1) are divided between Linac1 (four sections) and Linac2 (three sections) and are used upstream and downstream of BC1. S0A, S0B and the CERN sections are traveling wave (TW) structures operating in the $2 / 3 \pi$ mode per cells and coupled on axis. Seven Elettra-sections (S1-S7, blue colored in Fig.1) make up Linac3 (two sections) and Linac4 (five sections) and are used upstream and downstream of BC2. They are backward traveling wave (BTW) structures operating in the $3 / 4 \pi$ mode and magnetically coupled. Detailed information for all three types of the accelerating sections is given in Table (2.1)

Table (2.1) Parameters of the accelerating sections

\begin{tabular}{|l|c|c|c|}
\hline & S0A, S0B sections & $\begin{array}{c}\text { CERN sections } \\
(\mathrm{C} 1-\mathrm{C} 7)\end{array}$ & $\begin{array}{c}\text { ELETTRA sections } \\
(\text { S1-S7) }\end{array}$ \\
\hline Iris radius $[\mathrm{mm}]$ & $10.3^{\mathrm{S}} / 9.73^{\mathrm{D}}$ (average) & $(18-25) \pm 0 . .010^{\mathrm{M}}$ & $5.0^{\mathrm{S}}$ \\
\hline Outer radius $[\mathrm{mm}]$ & $39.1^{\mathrm{S}} / 39^{\mathrm{D}}$ & $(82-85) \pm 0 . .010^{\mathrm{M}}$ & $39^{\mathrm{D}}$ \\
\hline Cell length $[\mathrm{mm}]$ & $33.33^{\mathrm{S}}$ & $33.321 \pm 0.008^{\mathrm{M}}$ & $37.5^{\mathrm{S}}$ \\
\hline Gap length $[\mathrm{mm}]$ & $30.43^{\mathrm{S}}$ & $28.321 \pm 0 . .012^{\mathrm{M}}$ & \\
\hline Number of cells & & & \\
\hline
\end{tabular}




\begin{tabular}{|l|c|c|c|}
\hline $\begin{array}{l}\text { (without input and } \\
\text { output cell) }\end{array}$ & $93^{\mathrm{D}}$ & $135^{\mathrm{D}}$ & $162^{\mathrm{D}}$ \\
\hline Total length [mm] & $3200^{\mathrm{D}}$ & $4500^{\mathrm{D}}$ & \\
\hline $2 / 3 \pi$ mode $[\mathrm{MHz}]$ & $2998.05^{\mathrm{S}} / 2997.747^{\mathrm{M}}$ & & \\
\hline $3 / 4 \pi$ mode $[\mathrm{MHz}]$ & & & $2997.74^{\mathrm{M}}$ \\
\hline $\mathrm{Q}$ & $14100^{\mathrm{S}} / 14100^{\mathrm{M}}$ & & $15700^{\mathrm{S}} / 11700^{\mathrm{M}}$ \\
\hline $\mathrm{R}_{0}[\mathrm{M} \Omega / \mathrm{m}]$ & $68^{\mathrm{S}} / 67.1^{\mathrm{D}}$ & & $97^{\mathrm{S}} / 72^{\mathrm{M}}$ \\
\hline $\mathrm{R}_{0} / \mathrm{Q}[\mathrm{k} \Omega / \mathrm{m}]$ & $4.8^{\mathrm{S}} / 4.76^{\mathrm{D}}$ & & $6.2^{\mathrm{S}}$ \\
\hline & & & \\
Filling time $[\mu \mathrm{s}]$ & $0.903^{\mathrm{M}}$ & & $0.757^{\mathrm{M}}$ \\
$\tau\left[\mathrm{N}_{\mathrm{p}}\right]$ & 0.603 & & $0.61^{\mathrm{M}}$ \\
$\mathrm{c} / \mathrm{v}_{\mathrm{g}}$ & $66.25^{\mathrm{S}} / 85^{\mathrm{M}}($ average $)$ & & 38 (constant) $^{\mathrm{M}}$ \\
$\alpha_{\text {init }}$ & 0.109 & & \\
\hline $\mathrm{P}_{0, \text { input }}[\mathrm{MW}]$ & $18^{\mathrm{D}}$ (input TW) $^{\mathrm{D}}[$ & & \\
\hline $\mathrm{E}[\mathrm{MV} / \mathrm{m}]$ & $16.6^{\mathrm{D}}$ & $10^{\mathrm{D}}$ & \\
\hline Total gain $[\mathrm{MV}\}$ & $50^{\mathrm{D}}$ & $45^{\mathrm{M}}$ & $120^{\mathrm{M}}$ \\
\hline
\end{tabular}

${ }^{\mathrm{s}}$ Electromagnetic simulation (for S0A, SOB SUPERFISH has been used over 40 cells). ${ }^{\mathrm{M}}$ Measured [Ref.9]

${ }^{\mathrm{D}}$ Technical design value or estimated

\subsubsection{Longitudinal wake potentials}

An electron moving at the speed of light $c$ through the accelerating structures excites the fields, called wakefields, that other electrons, moving behind at distance $s$, will experience. The longitudinal wake function $w(s)$ is the voltage loss experienced by the test electron, typically given in units $[\mathrm{V} / \mathrm{C}]$ for a single object (accelerating section in our case) or in $[\mathrm{V} / \mathrm{C} / \mathrm{m}]$ per unit length. The wake is zero if the test electron is in front $(s$ $<0$ ). For a longitudinal charge distribution $\lambda_{z}$, the voltage gain for a test electron from the wakes of all electron in front of it is given by the wake potential [10]:

$$
W(s)=-\int_{s}^{\infty} w\left(s-s^{\prime}\right) \lambda_{z}\left(s^{\prime}\right) d s^{\prime}
$$

An exact calculation of the wake function for an accelerating section consisting of an array of cells is difficult, but it was shown that obtaining the short-range wake numerically and fitting it to a simple function, one can obtain a result that is valid over a large range of $s$ and over a useful range of parameters [11]:

$$
w(s)=A \frac{Z_{0} c}{\pi a^{2}} \exp \left(-\sqrt{s / s_{0}}\right)
$$

where $Z_{0}=377 \Omega$ and $A$ and $s_{0}$ are fitting coefficients. Typically $A \approx 1$ and $s_{0}$ is close to the value given by the following expression [11]:

$$
s_{0}=0.41 \frac{a^{1.8} g^{1.6}}{L^{2.4}}
$$


where $a, g, L$ are defined in Figure (2.2).

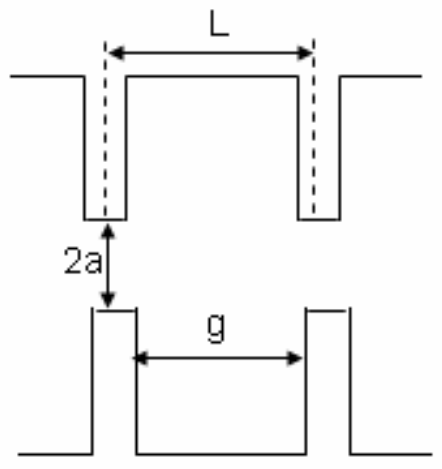

Figure (2.2). A schematic of the geometry for the SOA, SOB and CERN accelerating sections. All dimensions are given in Table 2.

Eq. (2.10) applies to a steady state situation, but it takes an approximate distance $a^{2} / 2 \sigma_{z}$ from the entrance of the accelerating section before the wake reaches the steady state. This is about $1 / 2 \mathrm{~m}$ for a $200 \mathrm{fs}$ bunch and $5 \mathrm{~mm}$ aperture. This estimates agrees with the numerical analysis performed in [12].

In what follows, the steady state wake functions obtained by fitting numerical calculations into (2.10) [Ref.12] will be used. For S0A and S0B acclerating structures one obtains:

$$
w(s)=380 \exp (-\sqrt{s(m m) / 1.28})\left[\frac{V}{p C m}\right]
$$

For the CERN acclerating structures:

$$
w(s)=311 \exp (-\sqrt{s(m m) / 1.38})\left[\frac{V}{p C m}\right]
$$

and for the ELETTRA acclerating structures:

$$
w(s)=1345 \exp (-\sqrt{s(\mathrm{~mm}) / 0.3})\left[\frac{V}{p C m}\right] \text { for } s<1 \mathrm{~mm}
$$

The wake potential in BTW structures is stronger than in TW structures because of the magnetic coupling that demands a smaller iris radius. However, the former produce a higher energy gain.

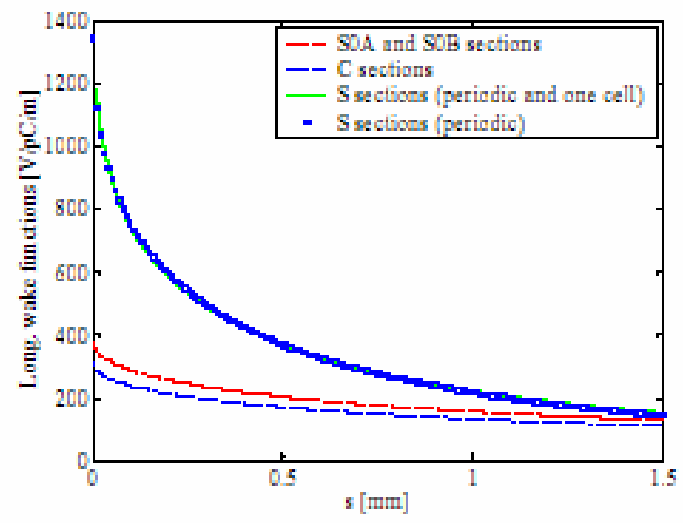


Figure (2.3). Longitudinal wake potentials for the accelerating sections used in the accelerator.

Using parabolic electron density distribution with $12 \mathrm{ps}$ length at the base and with the total charge of $1 \mathrm{nC}$ the wake potential induced by the SOA andSOB sections and linac 1 (for a total length of $\sim 24$ ) $\mathrm{m}$ is shown in Figure (2.4).

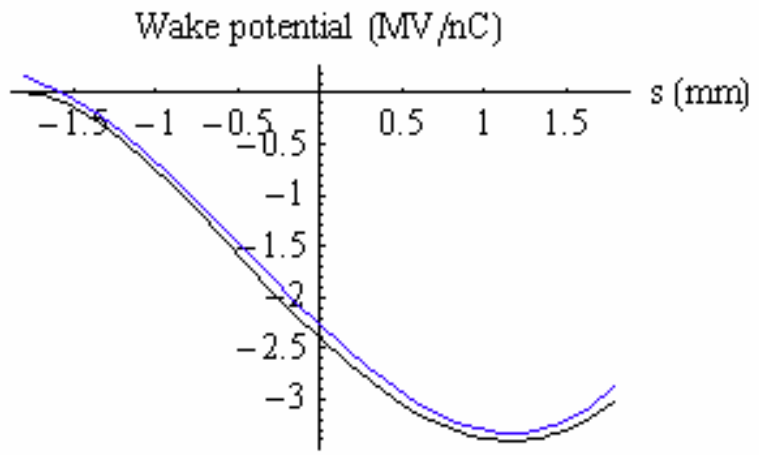

Figure (2.4). The wake potential obtained for $1 \mathrm{nC}$ bunch with the parabolic density distribution defined in the text moving through S0A and S0B and linac 1. The black line is the actual calculation and the blue line is the fit with a polynomial up to 3-rd power. The artificial vertical offset in the blue line is made to avoid the overlap of the two curves.

The result was fit by the 3-rd order polynomial:

$W(s)=\frac{k^{3} a_{3}}{3 !} s^{3}+\frac{k^{2} a_{2}}{2 !} s^{2}+k a_{1} s+a_{0}$

$a_{0} \approx-2.38 \mathrm{MV} / \mathrm{nC} ; a_{1} \approx-24.2 \mathrm{MV} / \mathrm{nC} ; a_{2} \approx 144 \mathrm{MV} / \mathrm{nC} ; a_{3} \approx 5000 \mathrm{MV} / \mathrm{nC}$

where the rf wave number $k$ is introduced for convenience in order to give dimensions of wake potential to the coefficients $a_{0}, a_{1}, a_{2}, a_{3}$. When $W(s) Q$, where $Q$ is the total bunch charge, is added to $U(s)$ in Eq.(2.1) it modifies all derivatives in (2.2) with addition of $Q k a_{1}$ into (2.2a), $Q k^{2} a_{2}$ into (2.2b) and $Q k^{3} a_{3}$ into (2.2c). The second derivative becomes zero when:

$$
U_{4}=-\frac{U_{0}+U_{1} \cos \left(\phi_{1}\right)-Q a_{2}}{16 \cos \left(\phi_{4}\right)}
$$

with this result, the first derivative can be written as:

$$
U_{(s=0)}^{\prime}=-k U_{1} \sin \left(\phi_{1}\right)+\frac{1}{4} k\left[U_{0}+U_{1} \cos \left(\phi_{1}\right)-Q a_{2}\right] \tan \left(\phi_{4}\right)+Q k a_{1}
$$

Using (2.13) in (2.6) one can obtain new expression for the energy chirp $h$. With zero second derivative, the cubic energy chirp defined by the third derivative in $(2.2 \mathrm{c})$ takes the following form:

$$
U_{(s=0)}^{\prime \prime \prime}=k^{3}\left[U_{1} \sin \left(\phi_{1}\right)+64 U_{4} \sin \left(\phi_{4}\right)+Q a_{3}\right]
$$


Now consider a numerical example using $k=2 \pi / 100 \mathrm{~mm}^{-1}, Q=1 \mathrm{nC}$ and a typical accelerator set-up with $\phi_{4}=0$ and $U_{(s=0)}^{\prime}=3.3 \mathrm{MV} / \mathrm{mm}$. Using in (2.13) the value of $a_{1}$ from (2.11), gives $U_{1} \sin \left(\phi_{1}\right)=-76.1 \mathrm{MV}$. This is to be compared with $Q a_{3} \approx 5000 \mathrm{MV}$ in (2.14), when $a_{3}$ from (2.11) is used. Thus, the cubic energy chirp is totally dominated by a contribution from the longitudinal wake potential. It will be shown later that the cubic energy chirp is responsible for appearance of the bifurcation in the phase space after the bunch compressor and this phase space distortion in turn leads to spikes in the peak current at the edges of the electron bunch. Therefore, it is desirable to minimize $a_{3}$. One way to achieve this is to use a density distribution other than the parabolic one considered so far. For example, a distribution with the linearly ramped peak current shown with the red line in Figure (2.5a) gives the wake potential with a significantly reduced cubic chirp $a_{3} \approx 890 \mathrm{MV} / \mathrm{nC}$ (see also the red line in Figure (2.5b).
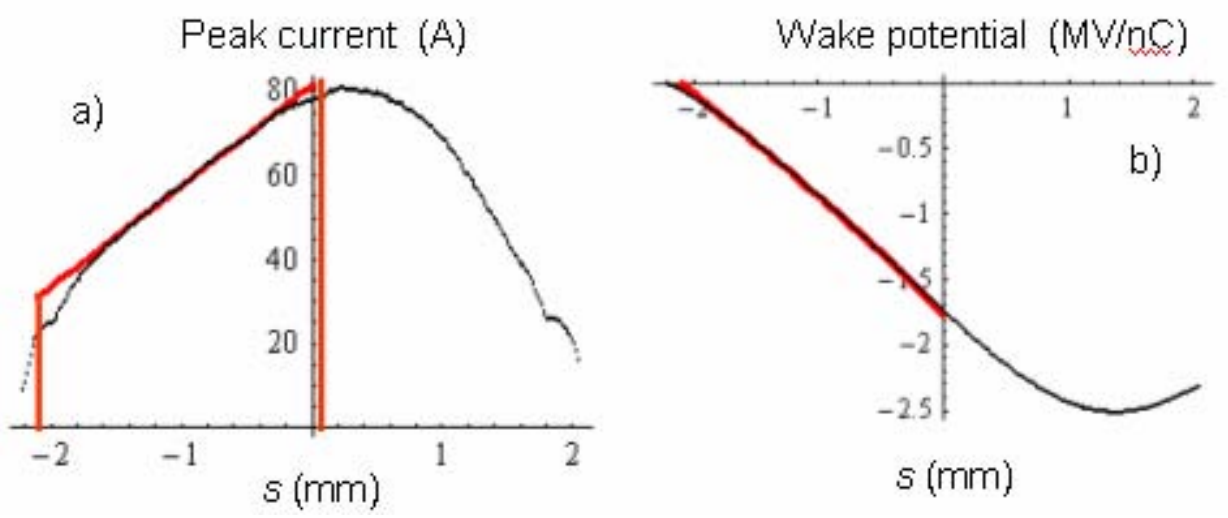

Figure (2.5). Density distribution with a linear ramped peak current (a) and a correspondent wake potential (b). The red line shows a desirable ideal distribution and its associated wake potential, while the black line shows the actual distribution obtained in simulations with the associated wake potential.

Certainly, the density distribution with sharp edges is unrealistic. The closest distribution and wake potential achieved at this stage of the injector studies is shown with the black line in Figure (2.5). The part under the red line in Fig.(2.5a) contains approximately $40 \%$ of the total charge containing under the black line.

\subsubsection{Coherent synchrotron radiation}

Coherent synchrotron radiation (CSR) in the bending magnets of the two bunch compressors plays a major role in the micro-fragmentation of the electron bunch. This effect will be considered later. In this paragraph the effect of synchrotron radiation is analyzed for a smooth electron density function when the emission of synchrotron radiation is coherent, at frequencies $\omega \leq c / \ell_{b}$, where $c$ is the speed of light and $\ell_{b}$ is the characteristic length of the scale of the bunch length [13]. This CSR causes variation of the electron energy along the electron bunch. In this section the importance of this effect is estimated. 
Following [14], two segments of the ultra-relativistic bunch, spaced at a distance $s \sim \ell_{b}$ ("tail" and "head") and moving in an $\operatorname{arc}(\mathrm{AB})$ as shown in Figure (2.6), are considered. The condition that the tail radiation at a point $\mathrm{A}$ will overtake the head of the bunch at a point $B$ is:

$$
s=\operatorname{arc}(A B)-\operatorname{line}(A B)=R \theta-2 R \sin (\theta / 2) \approx \frac{1}{24} \theta^{3} R
$$

This condition determines two important geometrical parameters (see, Figure (2.6)):

$\theta=3^{1 / 3} 2\left(\frac{s}{R}\right)^{1 / 3}$ and $d=\operatorname{line}(A B) \sin (\theta / 2)=2 R \sin ^{2}(\theta / 2)=3^{2 / 3} 2 s^{2 / 3} R^{1 / 3}$

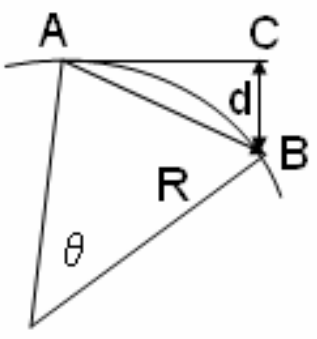

Figure (2.6). A geometrical diagram for an electron bunch motion in the bending magnet.

The magnitude of the transverse electric field which acts on the head particle can be estimated as the field of the charged line produced at the characteristic distance $d$ :

$$
E_{\perp} \cong \frac{2 Q \lambda_{z}}{d}
$$

Because this field was radiated at a point $\mathrm{A}$, its direction at a point $\mathrm{B}$ is perpendicular to the line(AC). Thus, the head particle experiences the acceleration force:

$$
F_{\|}(s) \cong e E_{\perp} \theta=\frac{2 e Q \lambda_{z}}{d} \theta=\frac{2 e Q \lambda_{z}}{3^{1 / 3} s^{1 / 3} R^{2 / 3}}
$$

In the case of the long magnet with bending angle:

$$
\varphi_{M} \geq\left(24 \frac{\ell_{b}}{R}\right)^{1 / 3}
$$

one can write for a rate of the electron bunch energy loss per unit length $z$ of the magnet:

$$
\frac{d E}{d z}=-\int_{-\infty}^{\infty} d s \lambda_{z}(s) F_{\|}(s)=-3^{2 / 3} \frac{N^{2} e^{2}}{R^{2 / 3} \ell_{b}^{4 / 3}}
$$

where $N$ is the number of particles per bunch and a uniform density distribution $\lambda_{z}=N / \ell_{b}$ is assumed. The uniform distribution arises in the second bunch compressor where CSR is strong. The condition (2.19) ensures that the magnet has a sufficient length that the radiation of the tail particles overtakes the head particles before the electron bunch leaves the magnet. In practice, the electron bunch moves inside the vacuum chamber that acts as a waveguide for the radiation. Not all spectral components of the CSR propagate in the waveguide and therefore the actual radiated energy is smaller than in a free space environment. For an estimation of the shielding effect of vacuum chamber the recipe suggested in [15] is used:

$$
\Delta E_{\text {shielded }} / \Delta E_{\text {free space }} \cong 4.2\left(n_{t h} / n_{c}\right)^{5 / 6} \exp \left(-2 n_{t h} / n_{c}\right), \quad n_{t h}>n_{c}
$$


Here $n_{t h}=\sqrt{2 / 3}(\pi R / \Delta)^{3 / 2}$ is the threshold harmonic number for a propagating radiation, $\Delta$ is the total height of the vacuum pipe, $n_{c}=R / \sigma_{c}$ is the characteristic harmonic number for a Gaussian longitudinal density distribution with the rms value of $\sigma_{c}$. For a uniform stepped density distribution $\sigma_{c} \cong l_{b} / 3.22$, with $l_{b}$ being the length of the distribution. The meaning of $n_{c}$ is that the spectral component of the radiation with harmonic numbers beyond $n_{c}$ is incoherent. Figure (2.7) shows the calculated effect of shielding for vacuum chamber with $\Delta=8 \mathrm{~mm}$.

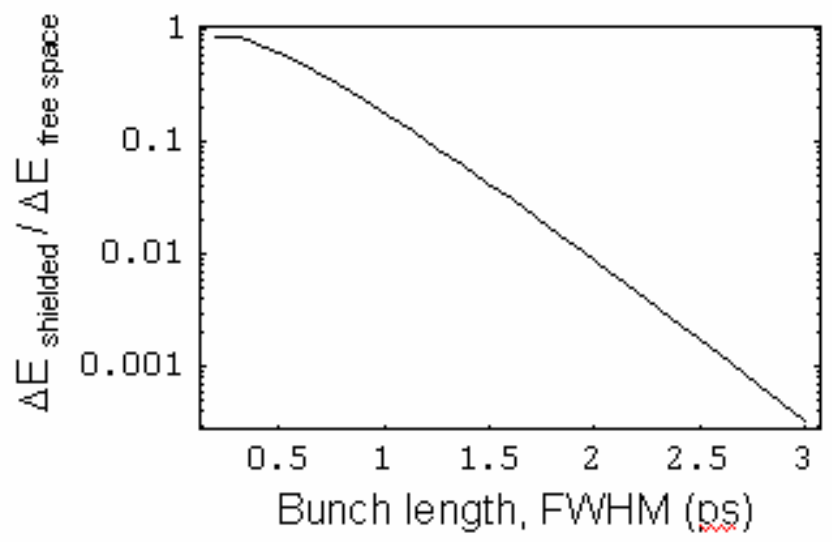

Figure (2.7). Suppression of CSR by the vacuum chamber shielding.

Because of shielding, the CSR is suppressed in $\mathrm{BC} 1$ (first bunch compressor), because the electron bunch length exceeds $2-3$ ps (in the medium and long bunch cases) and is only important in the 4-th magnet of $\mathrm{BC} 2$ (second bunch compressor) where the bunch length shrinks below 1 ps (at least in the medium bunch case). This magnet has a length of $0.5 \mathrm{~m}$ and a bending radius of $9.35 \mathrm{~m}$. Calculation using (2.19) results in an average energy loss per electron due to CSR in free space (i.e without shielding), and for a $0.7 \mathrm{ps}$ long electron bunch, of $340 \mathrm{keV}$. The arc angle of the magnet is approximately $25 \%$ less than that defined by condition (2.19) and, thus, the steady state formula (2.20) slightly overestimates the effect.

The energy variation along the electron bunch is estimated next and compared to the energy variation due to the wake potential. Using the CSR wake function [13], the wake potential is:

$$
W_{C S R}(s)[V / C / m]=-\frac{2}{3^{1 / 3}} \frac{e}{R^{2 / 3}} \int_{-\infty}^{s} \frac{1}{\left(s-s^{\prime}\right)^{1 / 3}} \frac{d \lambda_{z}\left(s^{\prime}\right)}{d s^{\prime}} d s^{\prime}
$$

We will use the electron density distribution with the flat top and smooth transitions at the edges with characteristic width of $\sim 2 \sigma_{0}$ described by erf function. The plot of this distribution is shown in Figure (2.8), with the assumption that the length of each transition occupies $\sim 10 \%$ of the main body. 


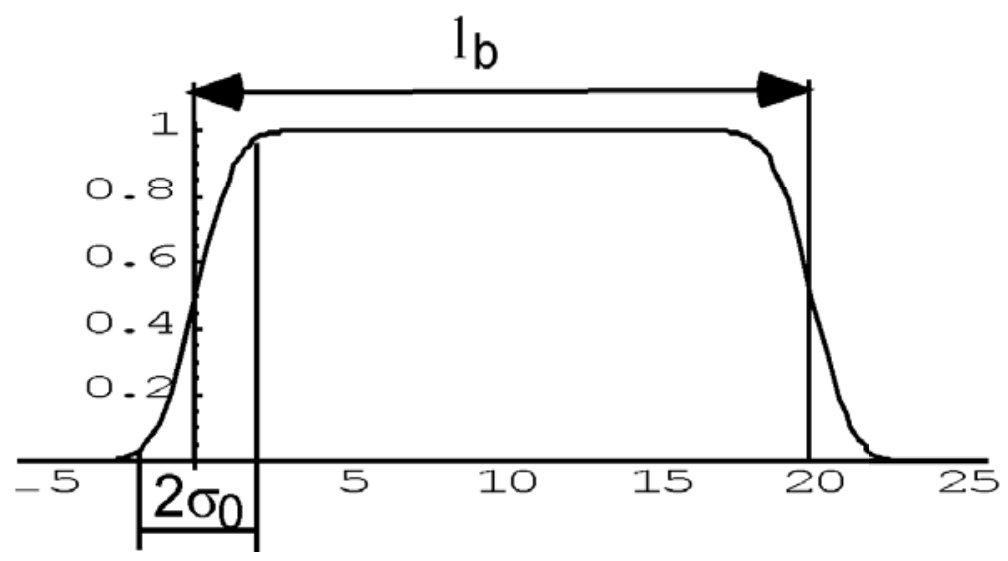

Figure (2.8). The longitudinal density of electrons.

Thus, instead of (2.22) one obtains:

$$
W_{C S R}(s)=-\frac{2}{3^{1 / 3}} \frac{e}{R^{2 / 3}} \int_{-\infty}^{s} \frac{1}{\sqrt{2 \pi} \sigma_{0}}\left(e^{-\frac{s^{\prime 2}}{2 \sigma_{0}^{2}}}-e^{-\frac{\left(s^{\prime}-l_{b}\right)^{2}}{2 \sigma_{0}^{2}}}\right) \frac{d s^{\prime}}{\left(s-s^{\prime}\right)^{1 / 3}},
$$

The integral (2.23) can be evaluated in analytical functions. The plot of $W_{C S R}(s) L_{M}$ is shown in Figure (2.9) for a bend magnet with the length $L_{M}=0.5 \mathrm{~m}$ and bending radius of $9.35 \mathrm{~m}$ is plotted, assuming $\sigma_{0} \approx 20 \mu \mathrm{m}$, i.e. $\sim 10 \%$ of the medium bunch length.

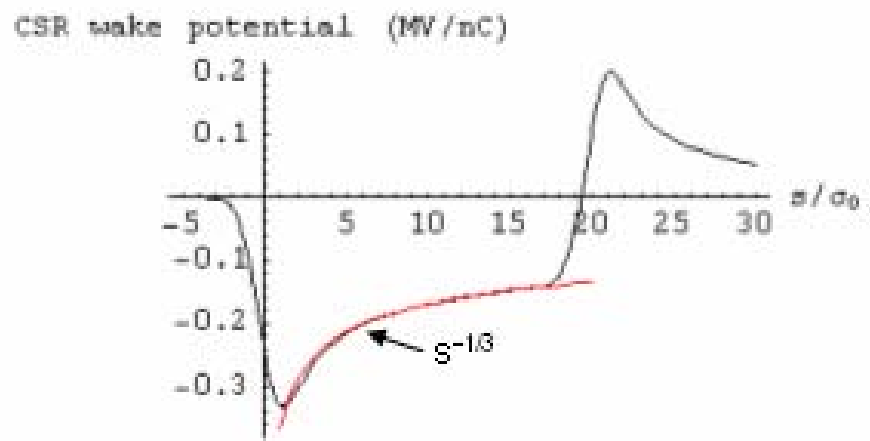

Figure (2.9) CSR wake potential for an electron longitudinal density distribution shown in Figure (2.8).

One can notice that $W_{C S R}(s) / \sim 1 / s^{1 / 3}$ over the entire length of the bunch excluding the edges and that the magnitude of the variation from head to tail is of the order of 0.15 MV. This is to be compared with $\sim 3$ MV in Fig.(2.4) or $\sim 2.5$ MV in Fig.(2.5). Therefore, this study concludes that the CSR affects electron bunches weaker than the wake fields. It is to be remembered that, so far, the CSR study applies to electron bunches with smooth electron density function. 


\subsubsection{Reverse tracking}

One of the problems faced by the accelerator optimization study was how to obtain, at the end of the accelerator, a distribution with constant peak current and slice medium energy along the electron bunch, referred to in this document as a "flat-flat distribution". This distribution is often at a premium in harmonic cascade FELs. This problem is considerably simplified by using the backward tracking technique demonstrated in the following paragraphs.

The basic premise, justified by analytical and computational studies, is that the output bunch configuration is largely pre-determined by the input bunch configuration and it must always be possible to find a unique electron density distribution at the beginning of the linac that produces a flat-flat distribution at the end of acceleration. If the effect of CSR in the bunch compressors on the medium slice energy can be ignored, then finding this distribution is relatively straightforward. One just needs to reverse the problem, i.e. to start at the end of the linac and move backward towards the beginning of accelerator. Eq.(2.24) shows that, for a given $\lambda_{z}$ and $w$, the electron energy at the end of a section of the linac, defined as $\delta_{f}$ (with $s_{f}$ being the electron coordinate taken with respect to the bunch center), can be found by the relationship between the electron energy $\delta_{i}$ and the coordinate $s_{i}$ at the beginning of the section:

$$
\delta_{f}\left(s_{f}\right)=\delta_{i}\left(s_{i}\right)+e U \cos \left(k s_{i}\right)-\int_{s}^{\infty} w\left(s-s^{\prime}\right) \lambda_{z}\left(s^{\prime}\right) d s^{\prime}
$$

For a relativistic beam the electron distribution function $\lambda_{z}$ does not change during acceleration, i.e. $s_{f} \equiv s_{i}$, and, therefore, Eq.(2.24) can be used to define $\delta_{i}\left(s_{i}\right)$ as a function of $\delta_{f}\left(s_{f}=s_{i}\right)$. Thus, beginning from a desirable particle distribution function at the end of the linac section, one can find that distribution function at the beginning of the linac section that will produce the desired one at the end.

A different situation arises in a bunch compressor when the electron coordinate at the end of the bunch compressor $s_{f}\left(\delta_{f}\right)$ is expressed as a function of the electron coordinates $s_{i}$ and energy $\delta_{i}$ at the beginning of the bunch compressor:

$$
z_{f}\left(\delta_{f}\right)=z_{i}+R_{56} \delta_{i}+T_{566} \delta_{i}^{2}+f_{C S R}\left(z_{i}, \delta_{i}\right) \quad \ldots
$$

$R_{56}, T_{566}$ are first and second order time-of-flight parameters and a function $f_{C S R}$ describes changes related to the CSR effects. It is convenient fact that, in the FERMI accelerator, the effect of CSR on the medium slice energy is small in BC2 and negligible in $\mathrm{BC} 1$; that is, its overall effect is much smaller than that of the wake fields. Thus, if one ignores CSR and assumes that the electron energy is not affected in the magnets of the bunch compressor, i.e. $\delta_{f} \equiv \delta_{i}$, then the electron coordinate at the beginning of the bunch compressor can be found using the electron coordinate at the end of the bunch compressor using Eq.(2.25). 
The above considerations justify a concept of reverse tracking demonstrated in Figure (2.10) that shows the results of simulations obtained with the LiTrack code [16]. Fig.(2.10a) shows the conventional forward particle tracking starting with the particle distribution at the end of the injector, while Fig.(2.10b) shows the reverse particle tracking starting with the distribution obtained at the end of the forward simulation (compare bottom left and top right sections of the plot). The result of this exercise confirms the validity of reverse tracking.
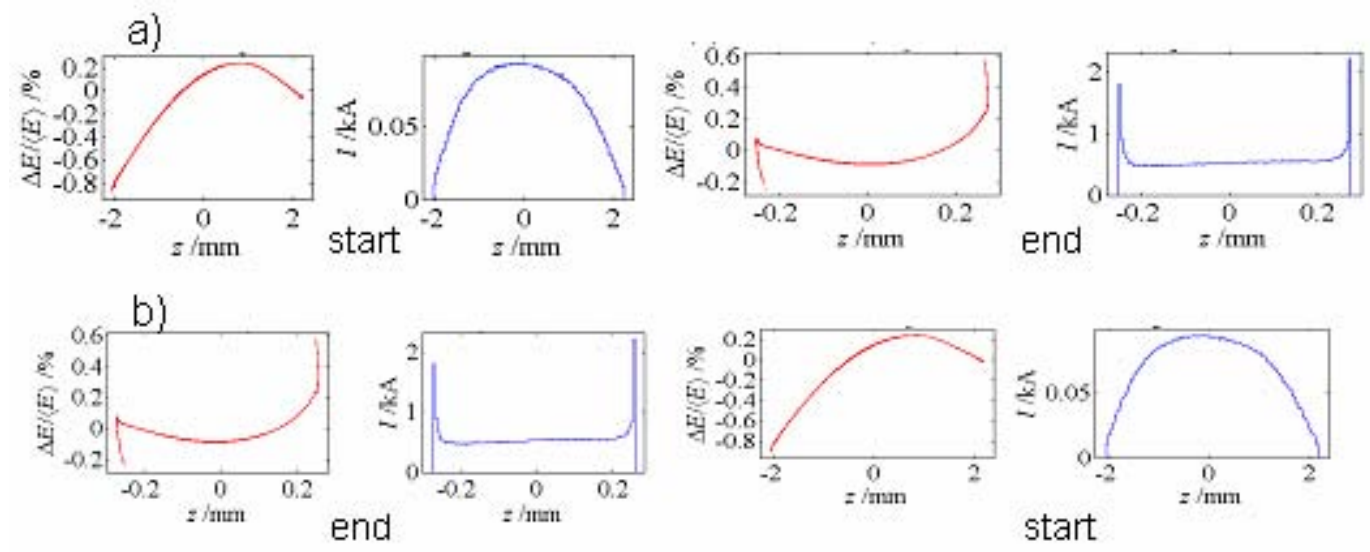

Figure (2.10). Plot a) shows forward particle tracking and plot b) shows reverse particle tracking.

In the next step, a desirable flat-flat distribution was set up at the end of the linac. Starting with this distribution and tracking it backward, the nearly linear ramped peak current of Figure (2.11) was obtained at the start of the linac. Here one can see that the magnitude of the peak current practically doubles from the head to the tail of the electron bunch. This is approximately the same ramped peak current distribution that was studied in paragraph (2.2.2). This result can be explained by the linear wake potential in Fig.(2.7) correspondent to the ramped peak current distribution.
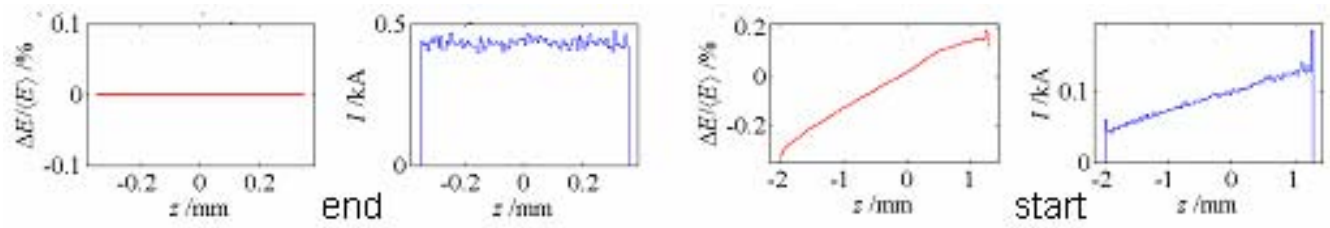

Figure (2.11). Reverse tracking beginning with flat-flat distribution at the end of the linac (start) and moving towards beginning of the linac (end).

Now we consider a condition that allows a conversion of a linear ramped peak current at the beginning of the accelerator into the flat distribution at the end of the accelerator. First we write formula (2.8) for a compression factor in an equivalent form:

$$
C=\frac{I_{a}}{I_{b}}
$$

where $I_{a}$ is the peak current after compression and $I_{b}$ is the peak current before compression. In our case we have: 


$$
I_{b}=I_{b 0}+I_{b}^{\prime} s \text { and } \quad I_{a}=I_{a 0}=\text { Const }
$$

Using (2.27) in (2.26) we obtain:

$$
\frac{1}{C}=\frac{I_{b 0}}{I_{a 0}}+\frac{I_{b}^{\prime}}{I_{a 0}} s,
$$

Thus a compression that decreases from the head to the tail of the electron bunch with linear dependence on $s$ produces a required transformation of the linear ramped peak current into the flat distribution. One can obtain this compression by adding a small quadratic component to the linear energy chirp before the bunch compressor which will lead to a modification of $h$ in (2.8):

$$
C=\frac{1}{1+\left(h_{0}+h^{\prime} s\right) R_{56}}
$$

where $h_{0}$ is defined by the linear energy chirp and $h^{\prime}$ is defined by the quadratic energy chirp. Comparing Eq.(2.29) and Eq.(2.28) we find for $h^{\prime}$ :

$$
h^{\prime} R_{56}=\frac{I_{b}^{\prime}}{I_{a 0}}
$$

This result can be easily generalized. For example, a distribution with the maximum peak current at the head of the bunch gradually reducing towards the tail can sometimes be beneficial for HC FEL, i.e. we may need $I_{a}=I_{a 0}-I_{a}^{\prime} s$. This distribution can be obtained with a slight modification to the quadratic energy chirp: $h^{\prime} R_{56}=I_{b}^{\prime} / I_{a 0}+I_{a}^{\prime} I_{b 0} / I_{a 0}^{2}$.

\subsubsection{Microbunching instability}

So far we considered collective effects with a typical scale of the order of the electron bunch length. We demonstrated that at this scale we can ignore CSR. We can also ignore longitudinal space charge effects (LSC) because they are weak in the relativistic electron bunch with a smooth density distribution. But at a scale much smaller than the bunch length both these effects gain significance. At this scale shielding is not important and any microstructures within the bunch will cause CSR as in a free space. Same microstructures will induce LSC forces which over the time will produce energy modulation of electrons within the electron bunch. Together, the LSC $[17,18]$ and the CSR [19,20,21] give rise to so-called microbunching instability studied before for LCLS $[22,23]$ and DESY XFEL. This is a rather fundamental instability that takes its roots in a shot noise of electrons. Shot noise is responsible for initial microbunching of electrons. The microbunching induces LSC forces and they produce energy modulation of electrons. Then, energy modulation is transformed into spatial modulation with increased magnitude of microbunching when electron bunch propagates magnets of bunch compressor. At the same time increased microbunching gives rise to increased intensity 
of CSR and it results in even more energy modulation and more microbunching and so on. The entire machine acts as gigantic amplifier of the initial noise in a direct similarity with the process of self amplified spontaneous emission in FELs. What one gets at the end is the electron beam with significant fragmentation in the longitudinal phase space.

Simulation of the microbunching instability with particle tracking codes requires a large number of macro-particles. For example, the microbunching amplitude due to shot noise in the electron beam with the peak current $I_{b}$ within the bandwidth $\Delta \lambda$ can be estimated:

$$
b=\sqrt{\frac{2 e c}{I_{b} \Delta \lambda}}
$$

For $I_{b}=75 \mathrm{~A}$ and $\Delta \lambda=10 \mu \mathrm{m}$ this formula gives $b=3.57 \times 10^{-4}$. Typically, the microbunching due to granularity of the distribution of macro-particles is much larger. For example, we calculate for a 6-picosecond long electron bunch (FWHM) with $10^{6}$ macroparticles:

$$
\left.b=\sqrt{\frac{6 \times 10^{-12} 2 c}{N \Delta \lambda}}=1.90 \times 10^{-2} \text { (at } \Delta \lambda=10 \mu \mathrm{m}\right),
$$

which is approximately 50 times larger than the real shot noise. At the same time, a practical limit for a number of macro-particles that one can use in the code Elegant [24] running on PC is about $2 \times 10^{6}$, which is still too small number and all the input noise is, thus, just an artificial numerical noise.

In order to get around of this problem we decided to develop another code IMPACT [25, see also Appendix A] that is capable of running at least $2 \times 10^{7}$ macroparticles on NERSC, the LBNLs multi processor computer cluster. We also pursued a completely different approach, namely a technique that follows the evolution of the distribution function using Vlasov's kinetic equation [26, see also Appendix B]. We call it Vlasov's solver. Ideally this technique is absolutely free from the computational noise, although some noise can be introduced there due to the final size of the grid where initial distribution function is defined. However, in practice, this noise can be easily kept below sensitivity level. Lastly, as a third option we used the linear theory [22] previously applied to LCLS project [23] to estimate the gain in the microbunching and in the energy spread caused by microbunching.

At the time of writing, three techniques mentioned above did not converge and the work is still in progress in attempt to identify a source of differences. In this paragraph we mainly describe results obtained with linear theory. Although it became apparent that a true result will likely be different because of the anticipation that linear model should fail at the high frequency end of the noise spectra, we want to present a simple transparent result which gives a correct assessment of the magnitude of the effect.

\subsubsection{Gain function}

We calculate the spectral dependence of the gain of the microbunching instability for the following set of machine and electron bunch parameters: uncorrelated energy spread $\sigma_{E}=2 \mathrm{keV}$, compression factor in $\mathrm{BC} 1=4$, electron beam initial energy $=100 \mathrm{MeV}$, 
electron beam energy at $\mathrm{BC} 1=220 \mathrm{MeV}$, linac length (from $100 \mathrm{MeV}$ to $220 \mathrm{MeV})=$ $30 \mathrm{~m}$, electron peak current after $\mathrm{BC} 1=350 \mathrm{~A}$. The result is shown in Figure (2.12).

Then, we moved bunch compressor to $130 \mathrm{MeV}$ (on a recommendation from Z. Huang) and used $7 \mathrm{~m}$ long linac and repeated gain calculations. Figure (2.13) shows the gain for this case. Both plots have on the horizontal axis the wavelength of the electron density modulation at the beginning of the accelerator. The reason why there is smaller gain of the microbunching instability when $\mathrm{BC} 1$ is at $130 \mathrm{MeV}$ is due to the fact that slippage effect in the chicane magnets is proportional to the product $R_{56} \sigma_{E} / E_{0}$, where $\sigma_{E}$ is the rms energy spread and $E_{0}$ is the equilibrium beam energy. Obviously, for a given compression, this product is almost two times larger for $E_{0}=130 \mathrm{MeV}$, than for $E_{0}=220$ $\mathrm{MeV}$ and instability is more "washed out" or "Landau damped" by the electron beam energy spread for a chicane at $130 \mathrm{MeV}$.

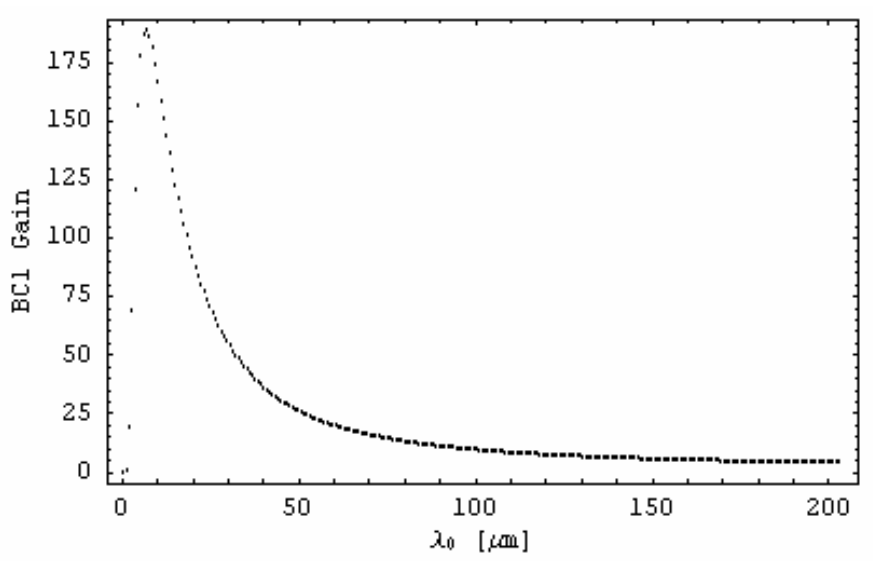

Figure (2.11). Spectral gain function of the microbunching instability calculated after BC1 located after $220 \mathrm{MeV}$ linac.

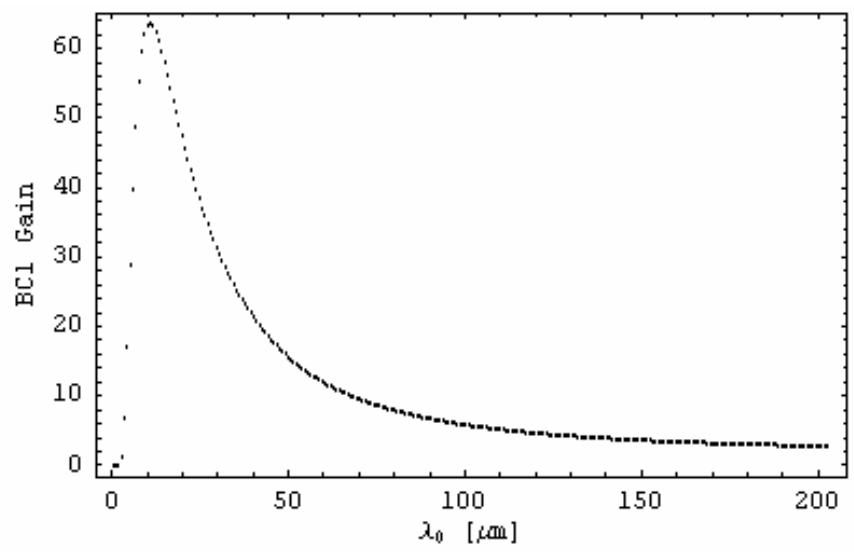

Figure (2.12). Spectral gain function of the microbunching instability calculated after $\mathrm{BC} 1$ located after $130 \mathrm{MeV}$ linac.

We present this result in order to demonstrate a potential for a reduction of the gain in the microbunching instability. But we decided not to pursue it since by placing $\mathrm{BC} 1$ at 130 $\mathrm{MeV}$ we restrict the flexibility in adjustment of the energy chirp before $\mathrm{BC} 1$. This could impact flexibility of the accelerator in operation with various beam configurations not 
foreseen at the moment. Thus, in what follows we show only results obtained for $\mathrm{BC} 1$ located at $220 \mathrm{MeV}$.

In the next step we calculate the spectral dependence of the gain of microbunching instability at the end of the linac using compression factor in the $\mathrm{BC} 2=$ 2.5 , electron beam energy at $\mathrm{BC} 2=600 \mathrm{MeV}$, linac length (from $220 \mathrm{MeV}$ to $600 \mathrm{MeV}$ ) $=50 \mathrm{~m}$, electron peak current after $\mathrm{BC} 2=900 \mathrm{~A}$ and linac length after $\mathrm{BC} 2$ including spreader area $=70 \mathrm{~m}$ (see, Figure (2.13)).

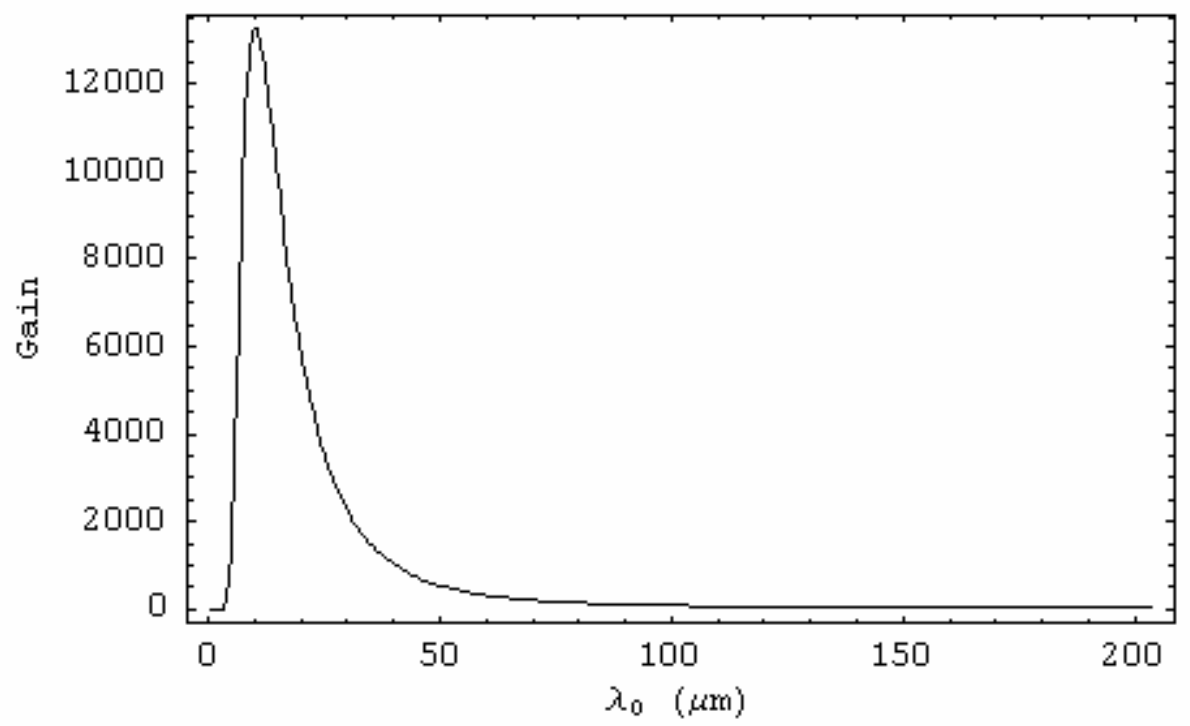

Figure (2.13) Spectral gain function of the microbunching instability at the end of the linac.

The above analysis shows that the most of the gain in microbunching instability occurs after $\mathrm{BC} 2$, i.e. after transformation of the energy modulation into the spatial modulation that takes place in $\mathrm{BC} 2$. It is possible to avoid that if we use only $\mathrm{BC} 1$ for all our needs for bunch compression. There are also additional advantages that we can gain from totally removing $\mathrm{BC} 2$. First, we would need to increase $R_{56}$ in $\mathrm{BC} 1$ (assuming that the energy chirp before $\mathrm{BC} 1$ remains unchanged). Second, a relative energy spread is significantly larger at $\mathrm{BC} 1$ than at $\mathrm{BC} 2$. Both these factors would contribute to increased "Landau damping" and suppression of the instability, which can be rather significant. Although, this is a very attractive option, we currently do not pursue it because difficulties in obtaining flat-flat distribution at the end of the linac due to strong longitudinal wake fields in linac 3 and linac 4. However, we do not think that all means were explored. One possibility is to use a special electron density distribution at the beginning of the accelerator such as to balance the effect of the wake fields. To find this distribution, one could possibly use a technique of the reverse tracking discussed in paragraph (2.2.4).

Finally we calculate slice energy spread in the electron bunch after the second bunch compressor assuming that the energy spread induced by microbunching instability will eventually become incoherent energy spread. We assumed initial shot noise with a constant spectra power and calculate initial bunching according to the formula 
$b^{2}=\frac{\sigma_{I}^{2}}{I_{b}^{2}}=\frac{2 e}{I_{b}} \Delta v$, where $\Delta v$ is the bandwidth. Then, we convolute it with spectral gain function $G_{E}(\lambda)$ shown in Fig.(2.13) to obtain:

$$
\left(\sigma_{E} / E_{0}\right)^{2}=\frac{2 e c}{I_{b}} \int G_{E}(\lambda)^{2} \frac{d \lambda}{\lambda^{2}}
$$

Here we used a substitution $\Delta v=c \Delta \lambda / \lambda^{2}$. This gives us a large number $\sigma_{E}=4.3 \mathrm{MeV}$ and in the next section we discuss how to get much smaller energy spread.

\subsubsection{Landau damping with the laser heater}

The gain of the microbunching instability is very sensitive to the uncorrelated energy spread in the electron beam. Typically, even a modest increase in the uncorrelated energy spread weakens the instability because of the "Landau damping" effect. Thus, "laser heater" was proposed in $[17,23]$ in order to have an efficient control over the uncorrelated energy spread with the ability to increase it manifolds beyond original small level. An actual description of the laser heater is given in the other report. Here we simply assume that the laser heater gives some additional energy spread which we add in quadrature to the existing uncorrelated energy spread at the beginning of the accelerator. In order to demonstrate the effect of the laser heater we calculated spectral gain function for a few different setting of the laser heater and plot them in Figure (2.14). It is seen here that the larger the energy spread added by the laser heater the more efficient is suppression of the gain at high frequency end of the spectra. We also calculated the uncorrelated energy spread at the end of the linac as a function of the energy spread added by the laser heater for a case of the medium bunch with the following beam and accelerator parameters:

e-beam energy at the beginning of the linac: $100 \mathrm{MeV}$

uncorrelated energy spread in the beam at the beginning of the linac: $2 \mathrm{keV}$

distance from the beginning of the linac to the first bunch compressor (BC1): $30 \mathrm{~m}$ e-beam energy at $\mathrm{BC} 1: 230 \mathrm{MeV}$

R56 of the BC1: $-0.026 \mathrm{~m}$

compression factor in the $\mathrm{BC} 1: 4$

e-beam peak current after $\mathrm{BC} 1: 350 \mathrm{~A}$

distance between $\mathrm{BC} 1$ and second bunch compressor (BC2): $50 \mathrm{~m}$

e-beam energy at BC2: $620 \mathrm{MeV}$

R56 of the BC2: $-0.016 \mathrm{~m}$

compression factor in the $\mathrm{BC} 2: 2.5$

e-beam peak current after BC2: $900 \mathrm{~A}$

and for a case of the long bunch with only changes of in the parameters listed below:

compression factor in the $\mathrm{BC} 1: 2$

e-beam peak current after BC1: $180 \mathrm{~A}$

R56 of the BC2: $-0.033 \mathrm{~m}$

compression factor in the $\mathrm{BC} 2: 2.7$ 
e-beam peak current after BC2: $500 \mathrm{~A}$

The results of both calculations are plotted in Figures (2.15) and (2.16).
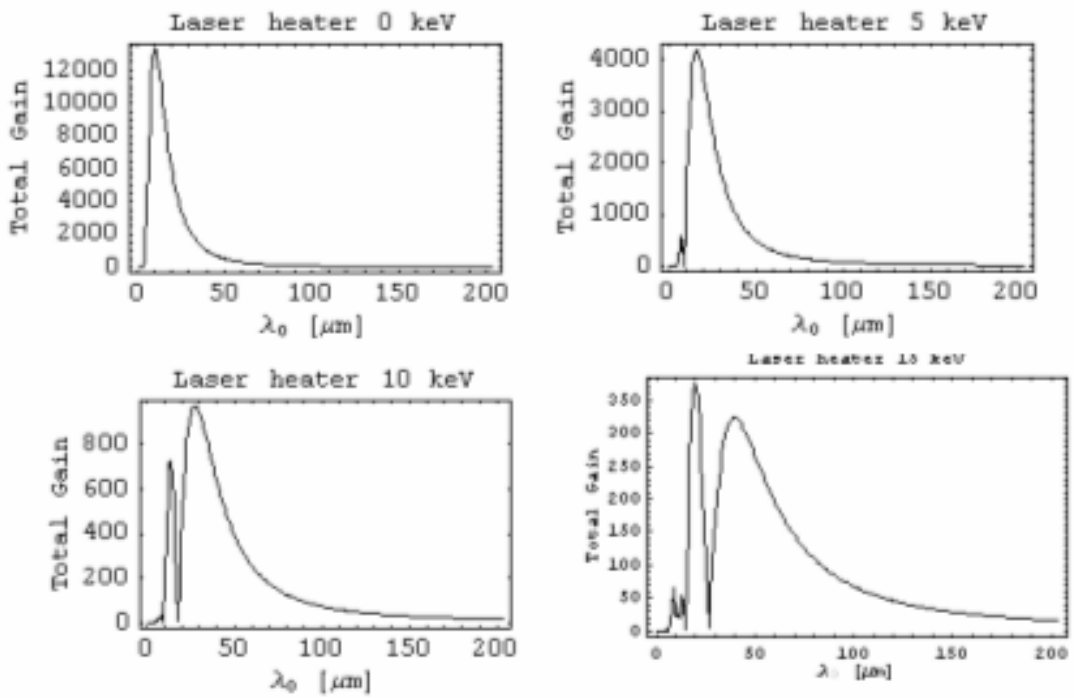

Figure (2.14) Spectral gain function of the microbunching instability at the end of the linac for various settings of the laser heater indicated above the plots.

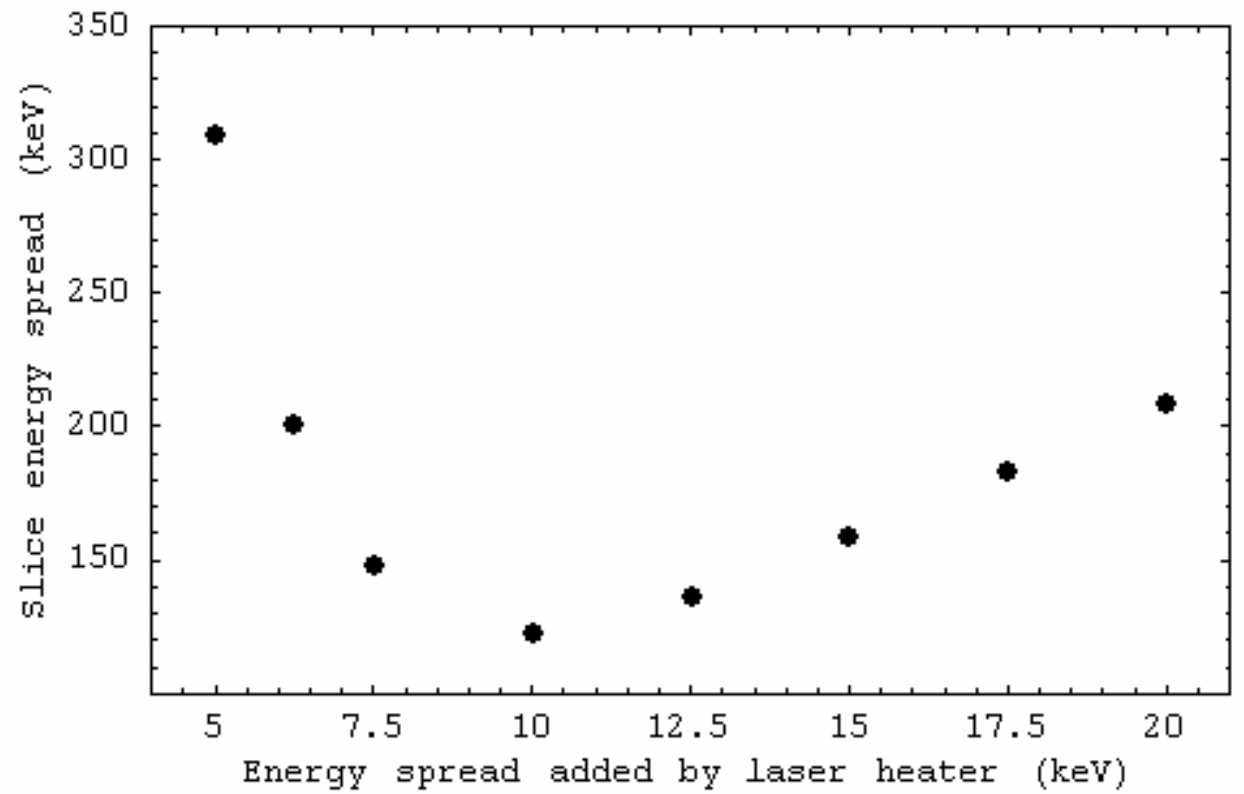

Figure (2.15). Medium bunch case. Uncorrelated slice energy spread after the second bunch compressor as a function of the energy spread added by the laser heater. 


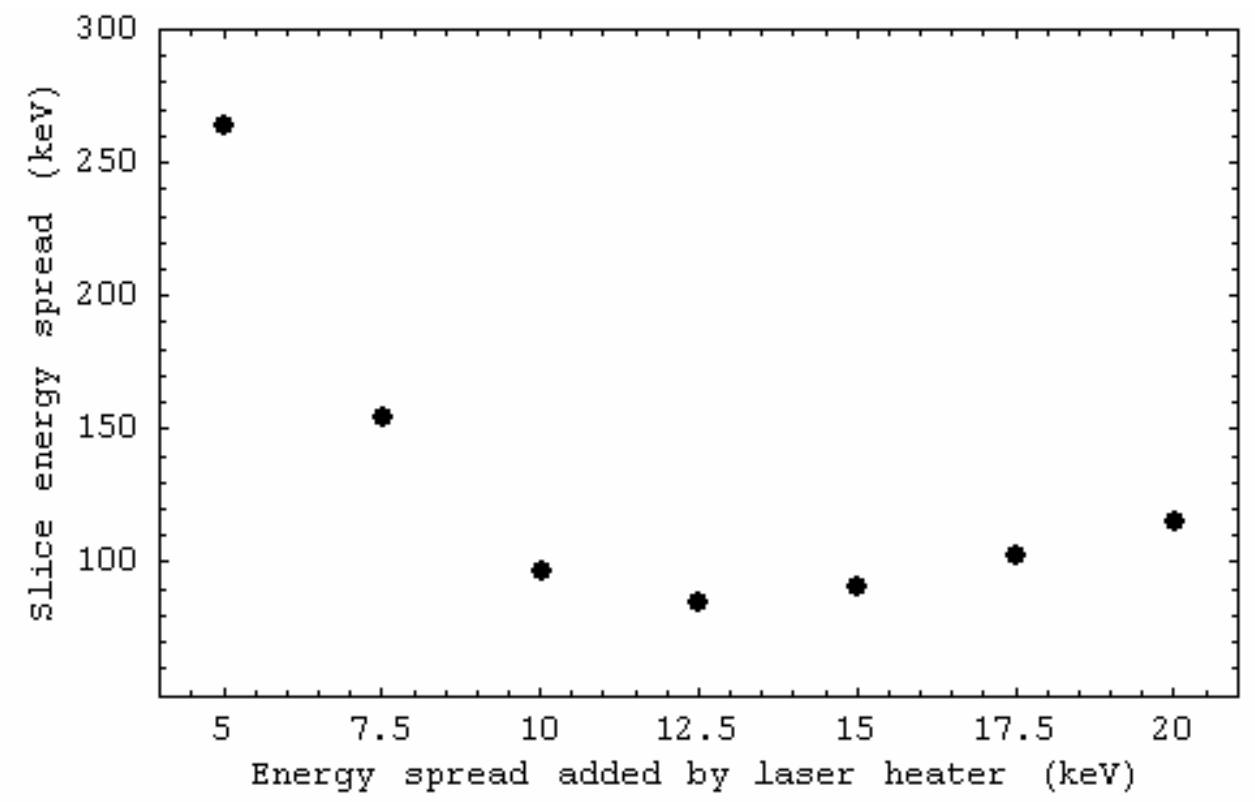

Figure (2.16). Long bunch case. Uncorrelated slice energy spread after the second bunch compressor as a function of the energy spread added by the laser heater.

Similar calculations performed with Vlasov's solver using initial electron beam with Gaussian energy distribution give somewhat different result shown in Figure (2.17) for the medium bunch case. In this calculation the evolution of the distribution function was traced beginning from $19 \mathrm{MeV}$ electron beam energy and to the end of the second bunch compressor at $600 \mathrm{MeV}$ electron beam energy. It was assumed the the electron density at the start corresponds to the peak current of 95 A with density fluctuations giving by the shot noise. Three different seeds generating the initial shot noise were used (except for the point at $11 \mathrm{keV}$, which was the average over two seeds). The error bars span the range of the results. Where the error bars are not visible the date range appeared to be smaller than the square box used in the plot. The solid line shows the expected energy spread in the absence of any collective effects assuming compression factor of 10 .

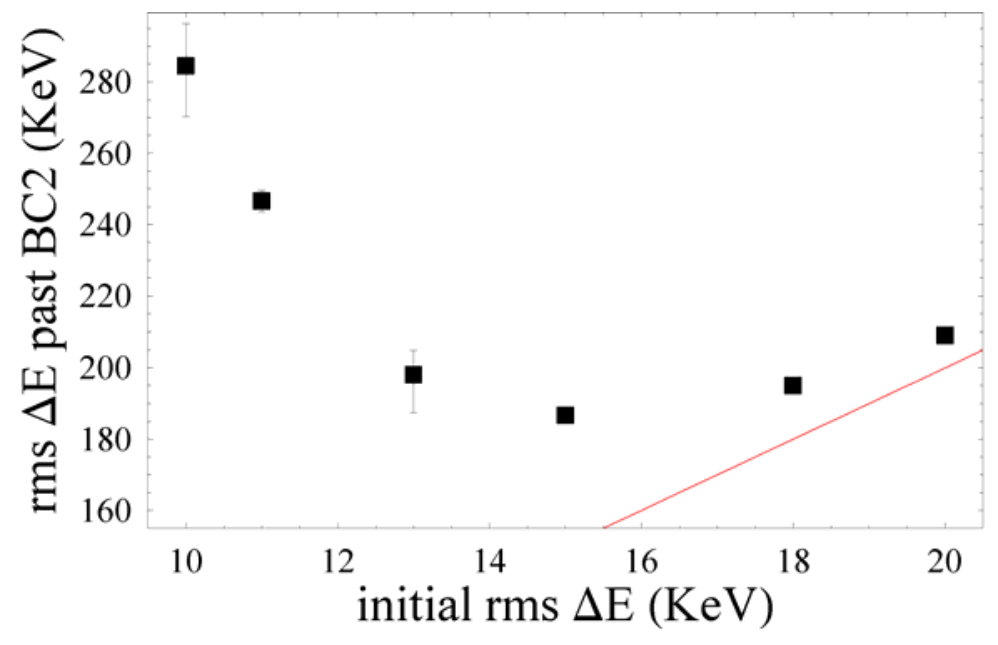


Figure (2.17). Medium bunch case. Uncorrelated slice energy spread as a function of the energy spread added by the laser heater calculated with Vlasov's solver. Solid line shows the expected energy spread in the absence of any collective effects assuming compression factor of 10

\section{Machine design}

In this paragraph we describe the lattice of the entire machine section by section and explain the logistics of this design. Figure (3.1) show Twiss functions of the entire accelerator, where we also indicate the locations of all major sections.

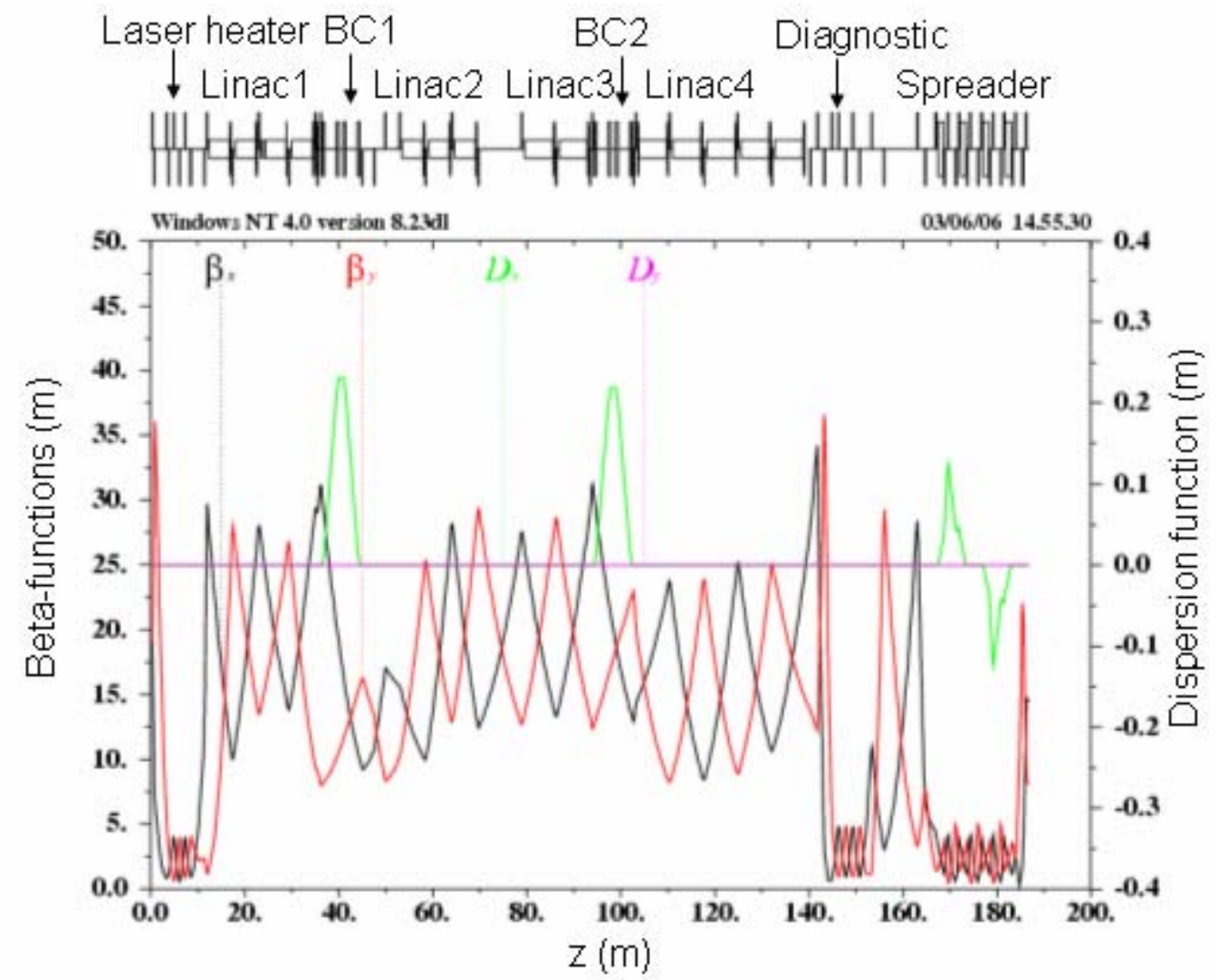

Figure (3.1) Twiss functions of the accelerator.

\subsection{Laser heater}

The accelerator begins with the laser heater. Laser heater lattice is also used to provide matching of the Twiss functions of the accelerator to the Twiss functions of the injector. The plot of the Twiss functions in this area is shown in Figure (3.2). Here we begin with $\beta_{x}=\beta_{y}=10 \mathrm{~m}$, where $\beta_{x}$ is the horizontal beta-function and $\beta_{y}$ is the vertical beta function. These beta-functions correspond to the matching condition in the short and medium bunch cases. However, laser heater lattice had been designed to be flexible in 
order to accommodate variations in the input beta-functions in the range of $3-40 \mathrm{~m}$. For example, the lattice $\beta_{x}=\beta_{y}=19 \mathrm{~m}$ is used to satisfy matching condition with the injector in a long bunch case. This lattice is actually shown in Fig.(3.1). In this chapter we do not provide the details regarding the laser heater because it will be done in a separate chapter and only mark a $2.6 \mathrm{~m}$ long drift where it is going to be located.

Besides being a tool for a control of the microbunching instability, laser heater is also an excellent diagnostic instrument. Therefore, we add a FODO channel after the laser heater for electron beam size measurements at various phases of betatron oscillations and plan to use it for emittance diagnostics. We are going to employ there removable screens in the locations indicated on the plot in Fig.(3.2). The betatron phase advances between first and second screen, second and third screen and third and fourth screen are $24^{\circ}, 79^{\circ}, 24^{\circ}$ in the horizontal plane and $72^{\circ}, 23^{\circ}, 57^{\circ}$ in the vertical plane.

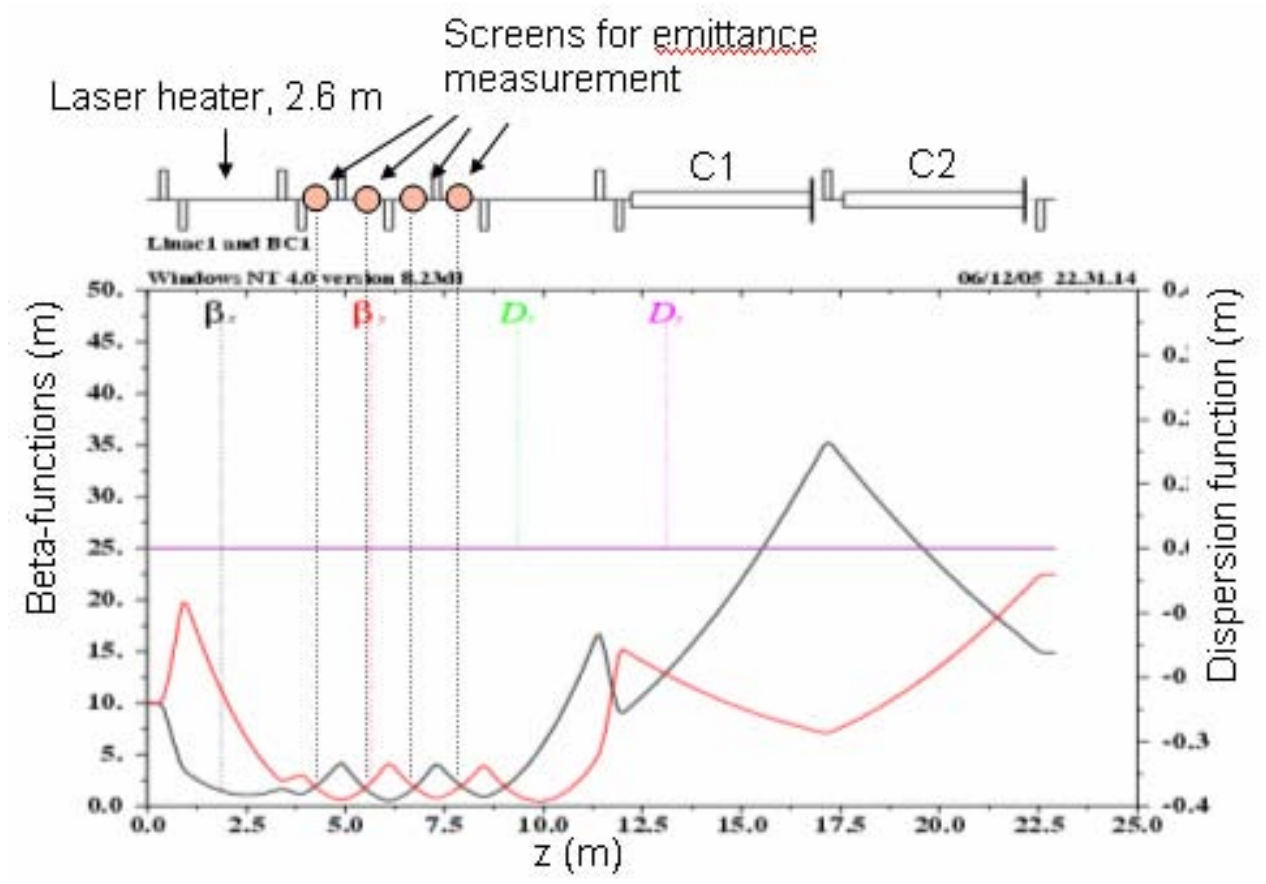

Figure (3.2) Twiss functions of the laser heater and adjacent diagnostic section.

\subsection{First bunch compressor}

A schematic of the bunch compressor is shown in Figure (3.3). It consists of a chicane built from four rectangular bending magnets. Due to the symmetry, this bunch compressor is a perfect achromat and ideally there must be no leakage of the dispersion function after the last bend. Nevertheless, it can happen due to the errors and trim quadrupoles (shown in Fig.(3.3) between first and second bends and third and fourth bends) are added for a fine tuning of the dispersion function. Dispersion is at its maximum in the middle of the chicane and, therefore, this is a convenient place for a collimator and energy monitor. Since the electron bunch typically has a significant 
energy chirp while it propagates the chicane, the collimator can be used to trim electrons in the head and tail of the electron bunch that could help to reduce peak current spikes at the bunch edges.

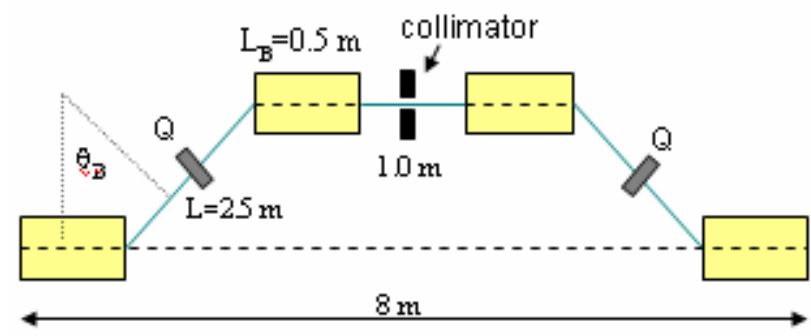

Figure (3.3) A schematic of the bunch compressor.

An important consideration for a design of the bunch compressor is the CSR. The CSR plays a role in the microbunching instability discussed in the paragraph (2.2.5) and also responsible for emittance excitation. Both these processes can be moderated with the appropriate design of the compressor lattice.

Although transverse microbunching radiative effects $[27,28]$ excite emittance directly, an indirect emittance excitation via longitudinal-to-transverse coupling typically dominates them. This coupling is characterized by the function:

$$
H=\left(\frac{1+\alpha_{x}^{2}}{\beta_{x}}\right) D_{x}^{2}+2 \alpha_{x} D_{x} D_{x}^{\prime}+\beta_{x} D_{x}^{\prime 2}
$$

where $\alpha_{x}, \beta_{x}$ are horizontal Twiss functions and $D_{x}, D_{x}^{\prime}$ are horizontal dispersion function and its derivative. (Here we assume a chicane in the horizontal plane.) Using $H$, we write for emittance contribution due to CSR:

$$
\delta \varepsilon_{x} \approx H(\delta E / E)^{2}
$$

where $\delta E / E$ is the spread of the energy losses caused by CSR, which is proportional to the value defined in (2.20). It is obvious from (3.2) that the lattice with small $H$ gives less emittance excitation. Since strongest CSR is expected in the 4-th bending magnet of the chicane where the electron bunch is the shortest, we pursue the lattice design with reduced $H$ in this magnet. It is seen from the reduction of the $\beta_{x}$ in the plot of Twiss function shown in Figure (3.4).

Now we would like to give the argument why we may not want to get the smallest possible $H$. While moving through chicane bend magnets, the electrons with different amplitudes of the betatron oscillations follow different paths with path lengths described by the following equation:

$$
\delta \ell(z)=\int_{0}^{z} \frac{x\left(z^{\prime}\right)}{R} d z^{\prime}=x_{0} \int_{0}^{z} \frac{C\left(z^{\prime}\right)}{R} d z^{\prime}+x_{0}^{\prime} \int_{0}^{z} \frac{S\left(z^{\prime}\right)}{R} d z^{\prime}
$$

Here $x_{0}, x_{0}^{\prime}$ are the electron coordinate and angle at the beginning of the chicane and $C(z), S(z)$ are cos-like and sin-like trajectory functions. It can be shown [26] that the rms 
value of $\delta \ell$ taken over the electrons in the any given slice of the electron bunch is related to the electron beam emittance through the function $H$, i.e.:

$$
\delta \ell_{r m s} \approx \sqrt{H \varepsilon_{x}}
$$

where $\varepsilon_{x}$ is the rms value of the electron beam horizontal emittance. Thus, the lattice with large $H$ spreads slice electrons more apart than the lattice with small $H$ and "washes out" the microbunching more effectively. In fact, without accounting for this effect, the gain of the microbunching instability would be significantly overestimated. This effect is very similar to the effect of the "Landau damping" due to the energy spread.

Because of the last argument, we designed $\mathrm{BC} 1$ lattice such as the magnitude of $H$ in the last bend of the chicane can vary at least within a factor of four. It will give us some flexibility to maneuver between such tasks as containing the emittance excitation due to CSR that benefits from smaller $H$ and containing energy spread growth due to the microbunching instability that benefits from larger $H$.

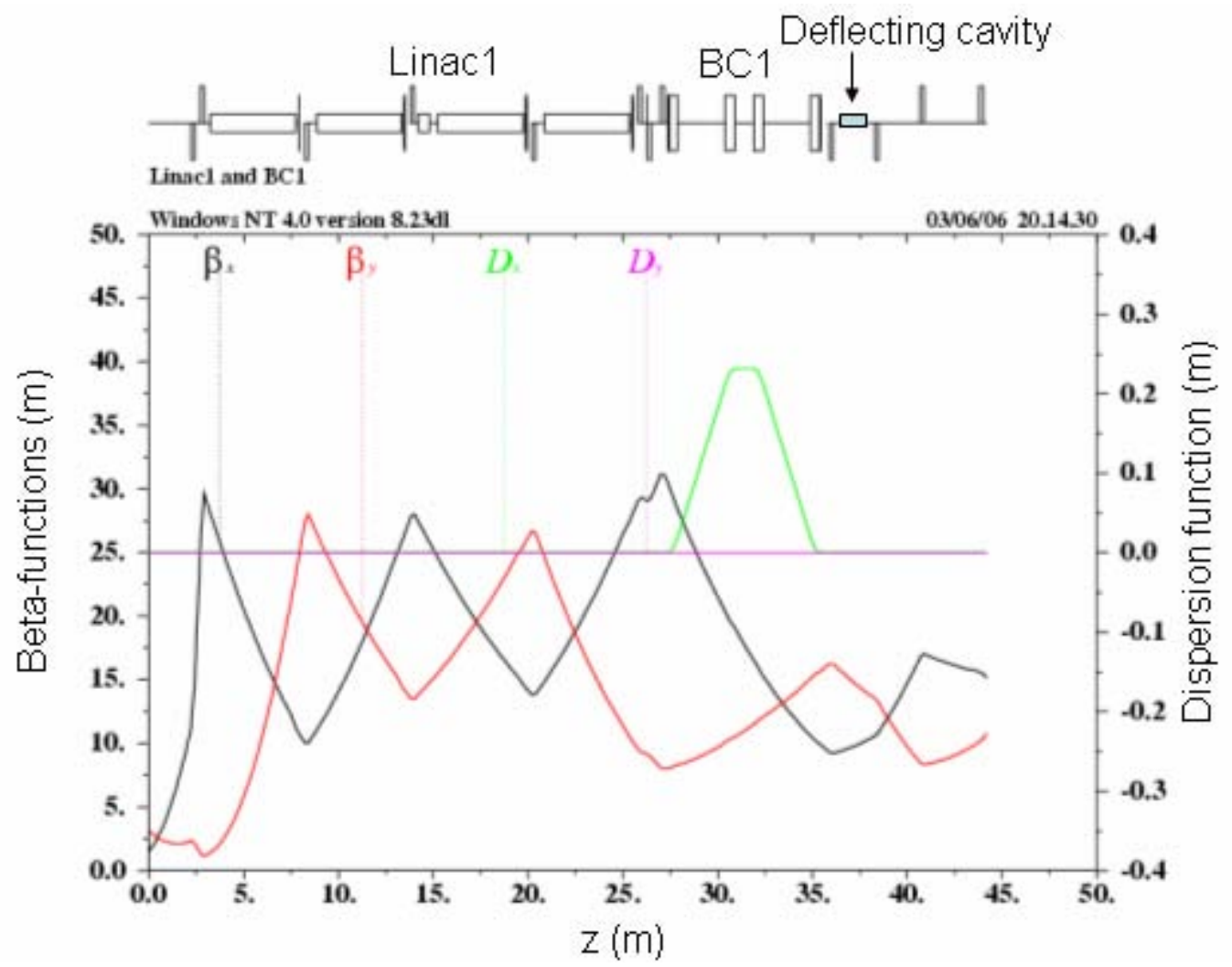

Figure (3.4). Twiss functions of the first bunch compressor and adjacent linac1.

Four quadrupole magnets after the chicane are used for matching of Twiss functions into downstream Linac 2. Between these magnets we reserved a space for a deflecting cavity to be used for emittance measurements and a space for temporal dump to be used during commissioning. 


\subsubsection{Bifurcation in the phase space and means to control it}

The first and second order time-of-flight parameters $R_{56}, T_{566}$ of the four bend magnet chicane shown in Fig.(3.3) can be calculated using the following expressions [29]:

$$
R_{56} \cong-\theta^{2}\left(2 L+\frac{4}{3} L_{B}\right) ; \quad T_{566} \approx-\frac{3}{2} R_{56},
$$

where $\theta$ is the bending angle, $L_{B}$ is the length of the magnet and $L$ is the distance between first and second bend magnets. We note that the bifurcation in the phase space, whose example is shown in Figure (3.5), depends on the second order time-of-flight parameter $T_{566}$ and on the cubic energy chirp defined by (2.14). But controlling the cubic chirp seems to be the only possible way to control the bifurcation since according to (3.5) the value of $T_{566}$ is totally defined by $R_{56}$.
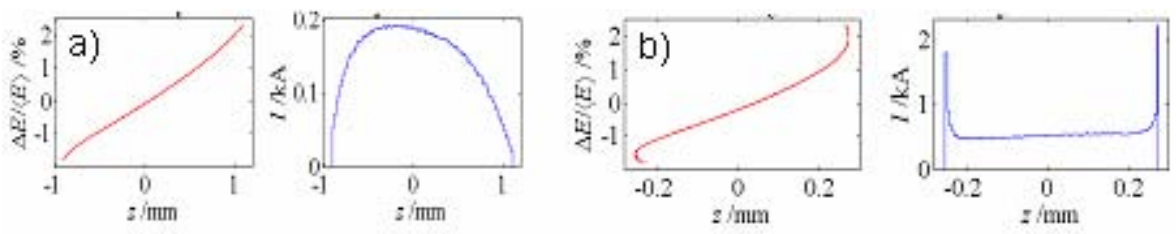

Figure (3.5). Electron distribution in the longitudinal phase space: a) before bunch compressor, b) after the bunch compressor. Cubic component in the energy chirp and second order time-of-flight parameter in the compressor are responsible for bifurcation in the phase space. This bifurcation gives rise to the spikes in the peak current at the edges of the electron bunch.

In the following plots we give the example how the variations in the cubic chirp in the initial electron density distribution affects the bifurcation of the electron distribution in the phase space after the bunch compressor. Figure (3.6a) shows the longitudinal phase space and the peak current at the beginning of the accelerator and Figure (3.6b) shows the same plots after the electron bunch compression. Here we see a strong bifurcation in the longitudinal phase space and the spikes in the peak current reaching $6 \mathrm{kA}$. The value of the cubic energy chirp in the beginning distribution is $-0.008 \mathrm{MeV} / \mathrm{mm}^{3}$. Then we repeated the same calculations keeping all the accelerator parameters fixed and changed only the sign and value of the cubic energy chirp in the initial distribution using -0.088 $\mathrm{MeV} / \mathrm{mm}^{3}$. The result is shown in Figure (3.7). The initial distribution in Fig.(3.7a) looks very similar to the initial distribution in Fig.(3.6a), but the output distribution is dramatically different. The spikes in the peak current at the bunch edges are now barely visible. We had similar experience in other cases. Every time we artificially made the cubic chirp in the initial distribution with the negative sign, we were able to get rid of huge spikes in the peak current in the output distribution. 
a)
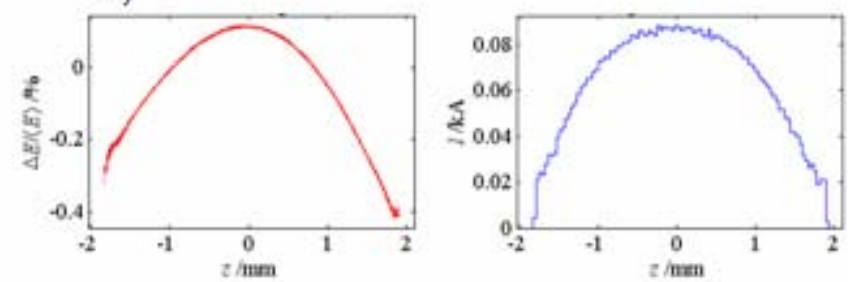

b)
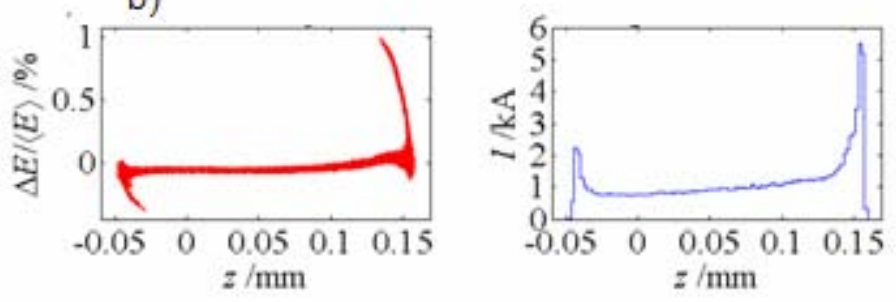

Figure (3.6). Longitudinal phase space and the peak current at the beginning of the accelerator a) and after electron bunch compression b). Cubic energy chirp in the initial distribution is $-0.008 \mathrm{MeV} / \mathrm{mm}^{3}$
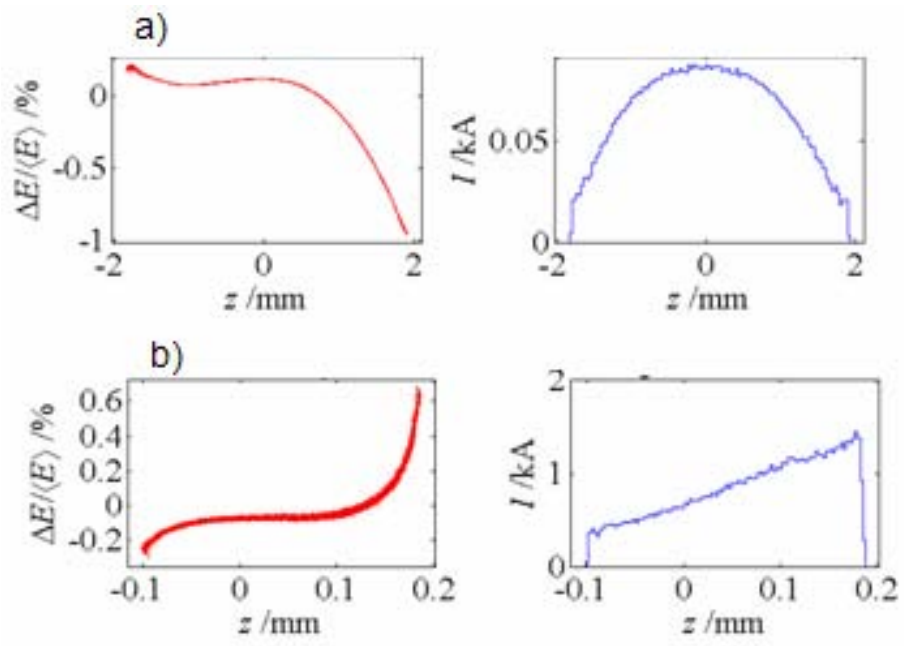

Figure (3.7) Longitudinal phase space and the peak current at the beginning of the accelerator a) and after electron bunch compression b). Cubic energy chirp in the initial distribution is $-0.02 \mathrm{MeV} / \mathrm{mm}^{3}$

We would like to point out that the distribution in Fig.(3.6b) is "flat" in the energy and in the peak current, while the distribution in Fig.(3.7b) is neither "flat" in the energy nor it is "flat" in the peak current. By using cubic energy chirp as a knob we were able to get either flat distributions or the distribution without spikes rather repeatedly, but not both features at the same time. Simply having just one knob is not enough. In the paragraph (2.2.2) we showed that one can change the cubic energy chirp by moving the phase of the $\mathrm{x}$-band harmonic cavity off-crest and/or by modifying initial peak current distribution and the longitudinal wake potential that depends on it. The former seems to be difficult in 
practice as the high voltage $\mathrm{x}$-band cavity would be needed, but the latter looks possibly realistic and must be studied in more details. At least the distribution with the ramped peak current discussed in paragraph (2.2.2) gives good results.

\subsection{Second bunch compressor}

A schematic of the second bunch compressor is exactly the same as the one for the fist bunch compressor shown in Figure (3.3). Twiss functions for this compressor are shown in Figure (3.8). Similar to the BC1, the lattice of this compressor is designed such as to reduce $H$ in the fourth bending magnet of the chicane. But, we also designed $\mathrm{BC} 2$ lattice such as the magnitude of $H$ in the last bend of the chicane can vary at least within a factor of four. There is no any specific physics related to BC2 that we had not already addressed in a discussion that we had for BC1.

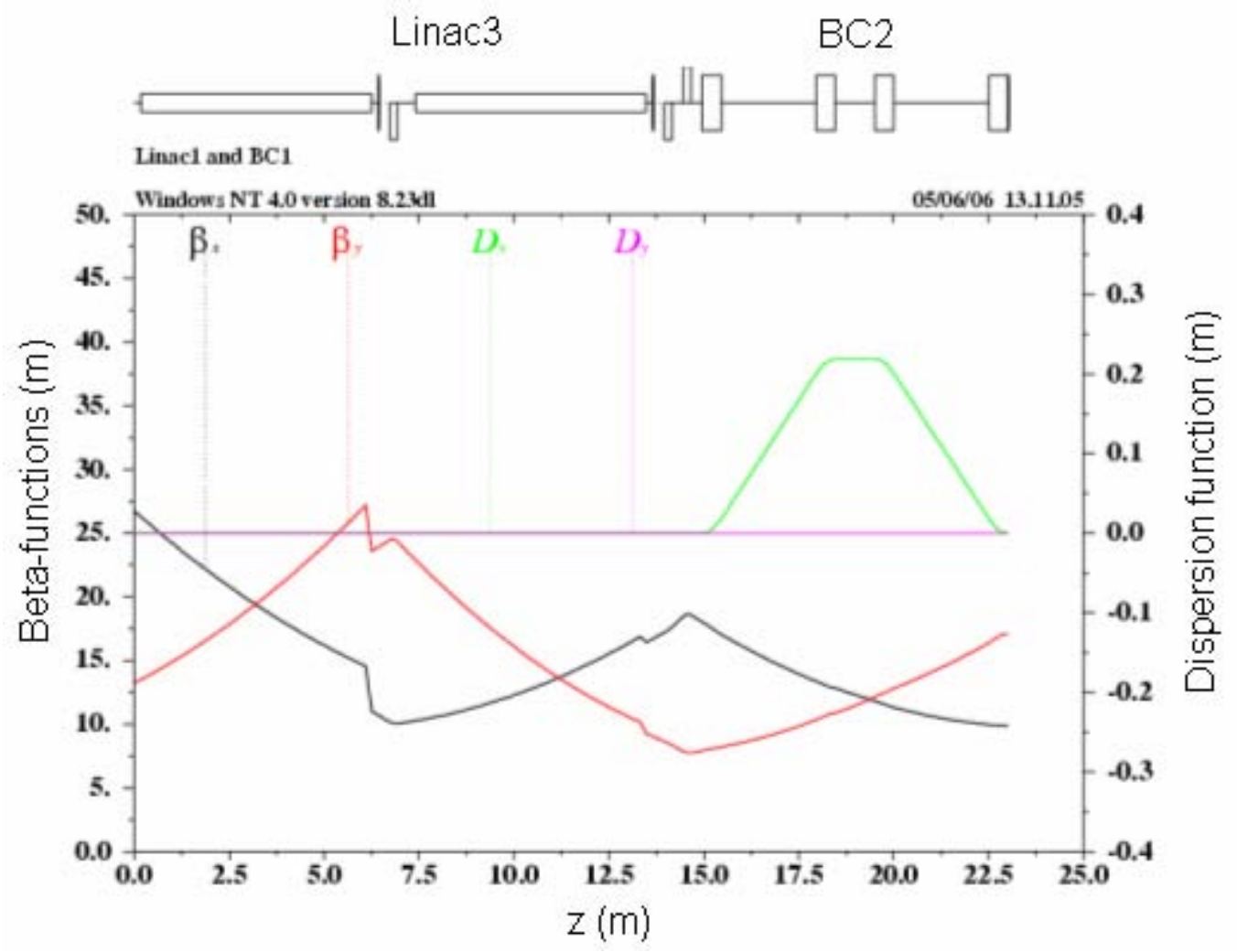

Figure (3.8) Twiss functions of the first bunch compressor and adjacent Linac 3.

\subsection{End of the linac and the spreader}

Twiss functions of this part of the machine are shown in Figure (3.9) The FODO channel after the Linac 4 is designed to provide approximately $135^{\circ}$ in the betatron phase advance in both planes for emttance measurements using the removable screens marked as solid circles on the plot. Downstream from the FODO channel we marked a possible convenient place for a deflecting cavity to be used for slice emittance measurement and 
for slice energy spread measurements at high dispersion points in the spreader. We actually designed two variants of the spreader: one with angular separation of two FELs and one with parallel separation of two FELs. A schematic of both variants is shown in Figure (3.10).

The important task for the lattice design of the spreader is to preserve the electron beam emittance while the electron bunch propagates the spreader. Basically, all

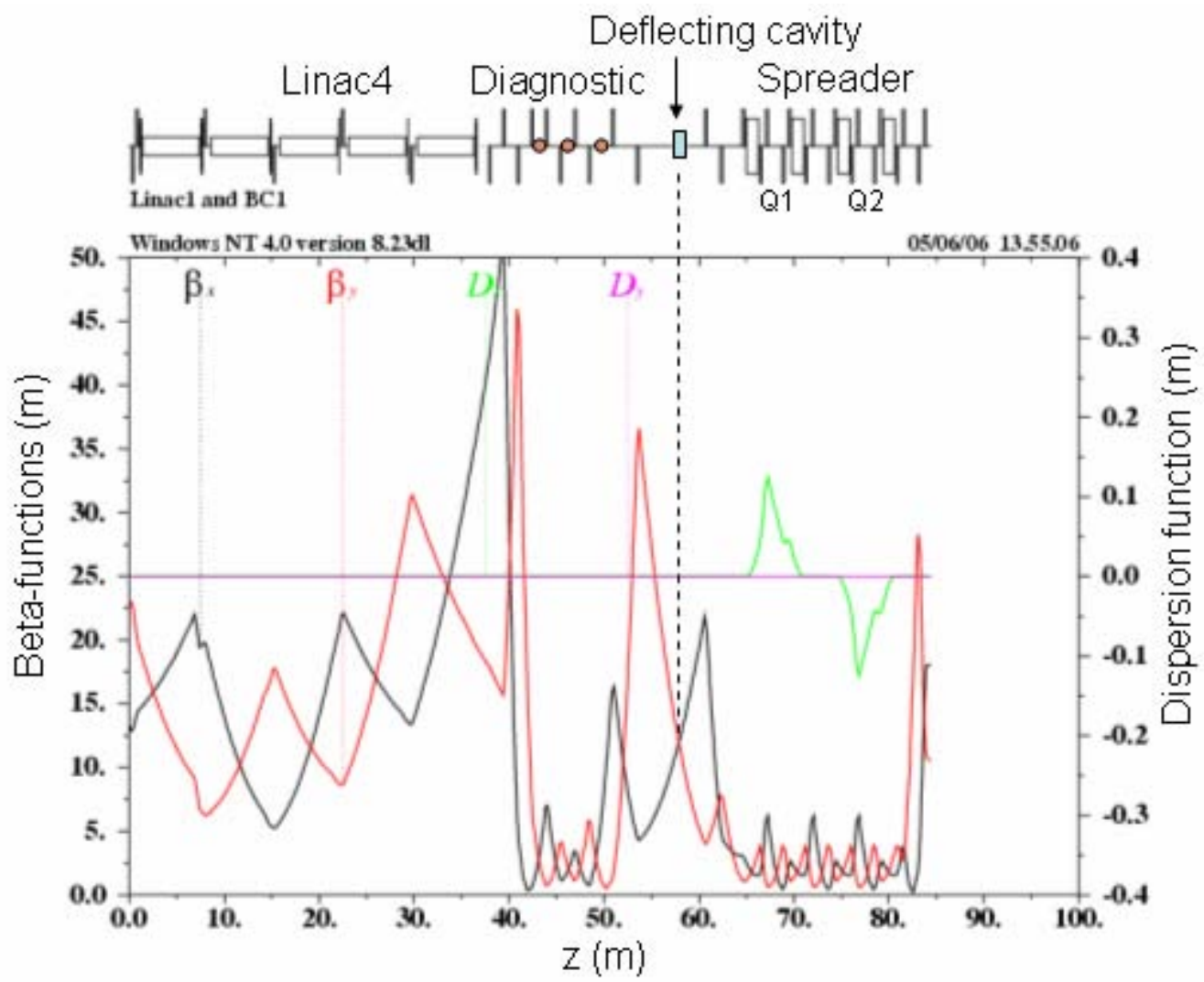

Figure (3.9) Twiss functions of the accelerator part beginning from the Linac 4 and ending by the entrance of the FEL.

a)

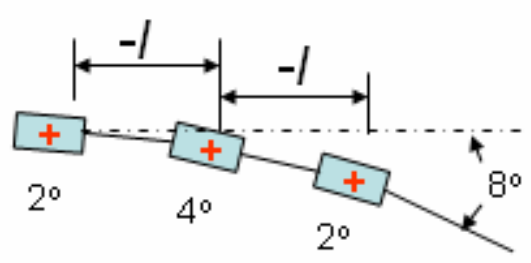

b)

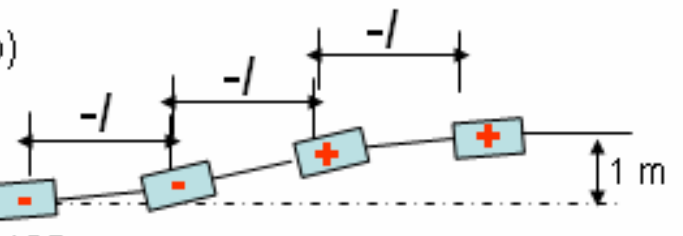

$3.125^{\circ}$

Figure (3.10) A schematic of the spreader: a) the spreader with angular separation and b) the spreader with parallel separation.

what is necessary is to contain the effect of CSR on the emittance. Here it is done by two means: i) by using the lattice with small $H$ function in the magnets and ii) by employing a scheme of self emittance compensation that we are going to explain now. 
Let $\delta_{E}$ be the relative energy loss due to CSR for an electron in the first bend magnet of the scheme shown in Fig.(3.10b). Then the kick to the electron trajectory due to this energy loss at the end of the first magnet can be written as:

$$
\delta x^{\prime}=D_{x}^{\prime} \delta_{E}
$$

where $D_{x}^{\prime}$ is the derivative of the dispersion function at the exit of the magnet. Since we use $-I$ transport between the magnets, then at the entrance of the second magnet this kick changes the sign and also the derivative of the dispersion function changes the sign. Here we also assume that electrons within the bunch are frozen while moving through the spreader and, therefore, the CSR causes the same amount of the energy loss in the second magnet as in the first magnet (as well as in the third and fourth magnets). Thus, we obtain at the exit of the second magnet for the kick of the electron trajectory:

$$
\delta x^{\prime}=-D_{x}^{\prime} \delta_{E}-D_{x}^{\prime} \delta_{E}=-2 D_{x}^{\prime} \delta_{E},
$$

where the first term in the middle part of the formula is due to the kick in the first bend and the second term is due to the kick in the second bend. After another $-I$ transport and another kick due to the CSR in the third magnet we get:

$$
\delta x^{\prime}=2 D_{x}^{\prime} \delta_{E}-D_{x}^{\prime} \delta_{E}=-D_{x}^{\prime} \delta_{E}
$$

Here again the first term in the middle part of the formula is due to two upstream kicks and the second term is due to the kick in the third bend. We note that the bending angle in the third and the fourth magnets is opposite to the bending angle in the third and the fourth magnets and, thus, $D_{x}^{\prime}$ after the third magnet has the opposite sign to $D_{x}^{\prime}$ after the first magnet. After another $-I$ transport and another kick, we obtain:

$$
\delta x^{\prime}=D_{x}^{\prime} \delta_{E}-D_{x}^{\prime} \delta_{E} \equiv 0,
$$

where the first term in the middle part shows the accumulative kick from three magnets. We see here, that at the end, the electron emerges with zero kick, and therefore, there must be no emittance excitation. In practice, however, the electron bunch is not completely frozen and there is a slight re-arrangement of electrons within the electron bunch. Certainly, this compromises a scheme, but not much. In all circumstances, the above described scheme is probably the best what one can do in order to contain the emittance excitation.

Similar analysis repeated for an angular spreader shows that the angle of the trajectory acquired in the central magnet should be twice as larger than the one in either of the side magnets.

Although both spreader schemes work in principle, our current preference is with the parallel spreader and this spreader lattice is shown in Fig.(3.9). We note that the spreader as it is shown in Fig.(3.9) has a small non-zero $R_{56}=2.4 \mathrm{~mm}$. However, it is easy to modify using quadrupoles Q1 and Q2 shown in the Fig.(3.9). These quadrupoles are separated by a unit transfer matrix and located near to the positive and negative peaks of the dispersion function. Thus, one can simultaneously change their gradients and produce a dispersion bump localized between the quadrupoles. By controlling this bump, one would be able to regulate the $R_{56}$ of the spreader making it to be exactly zero or any other reasonable value. In fact, we propose to keep it slightly positive in some cases in order to disperse the electrons in the spikes of the peak current at the edges of the electron bunch. Figure (3.11) illustrates the idea. Figure (3.11a) shows the original distribution, without energy variation in the main part and significant energy variation of 
the opposite sign in the tails. Figure (3.11b) shows what happens when we increase $R_{56}$ from $2.8 \mathrm{~mm}$ to $7 \mathrm{~mm}$. We disperse the electrons in the back spike more strongly and produce the anticipated reduction in the peak current.
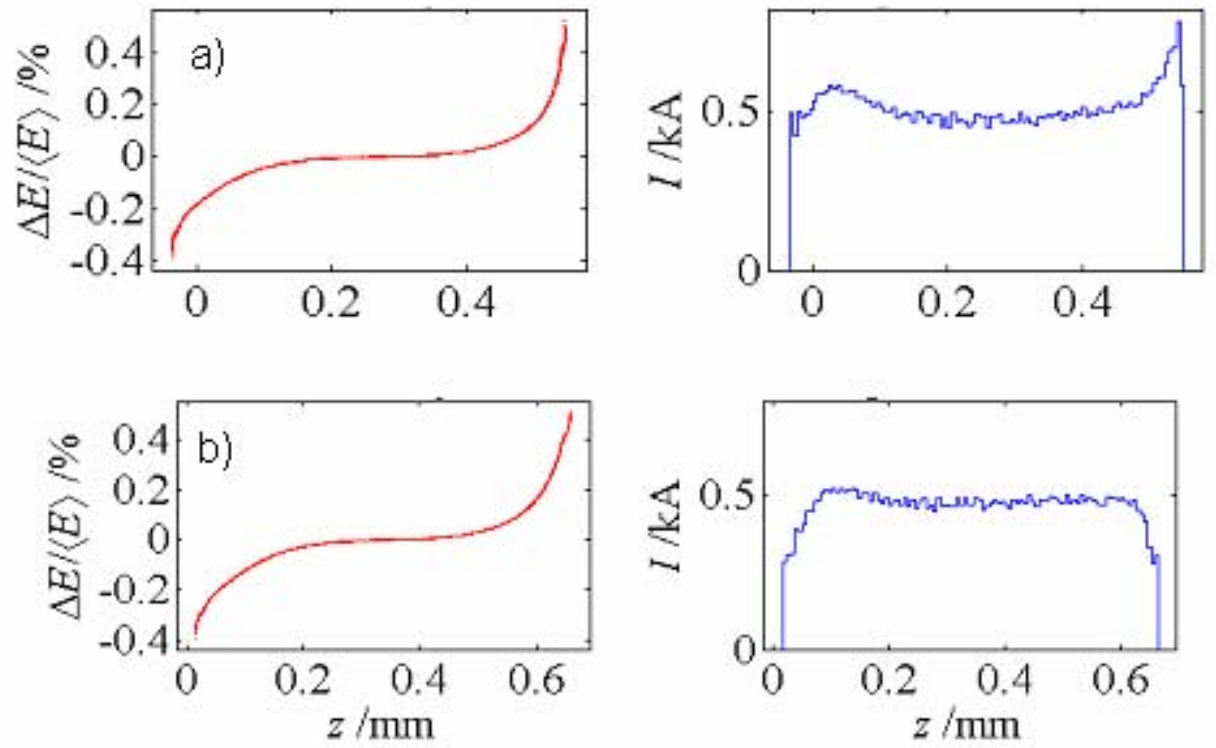

Figure (3.11). Illustration for the effect of the variation of the $R_{56}$ in the spreader: a) $\left.R_{56}=2.8 \mathrm{~mm}, \mathrm{~b}\right) R_{56}=7 \mathrm{~mm}$.

\section{Transverse beam dynamics}

The main issue of the study of the transverse beam dynamics is the preservation of a small normalized emittance along the Linac. The emittance may be so degraded by single particle dynamics effects as by collective effects. If we exclude the contribution from Coherent Synchrotron radiation (CSR), already discussed in Section 2.2.3, we may summarize the sources of emittance blow up as in Figure 4.1. 


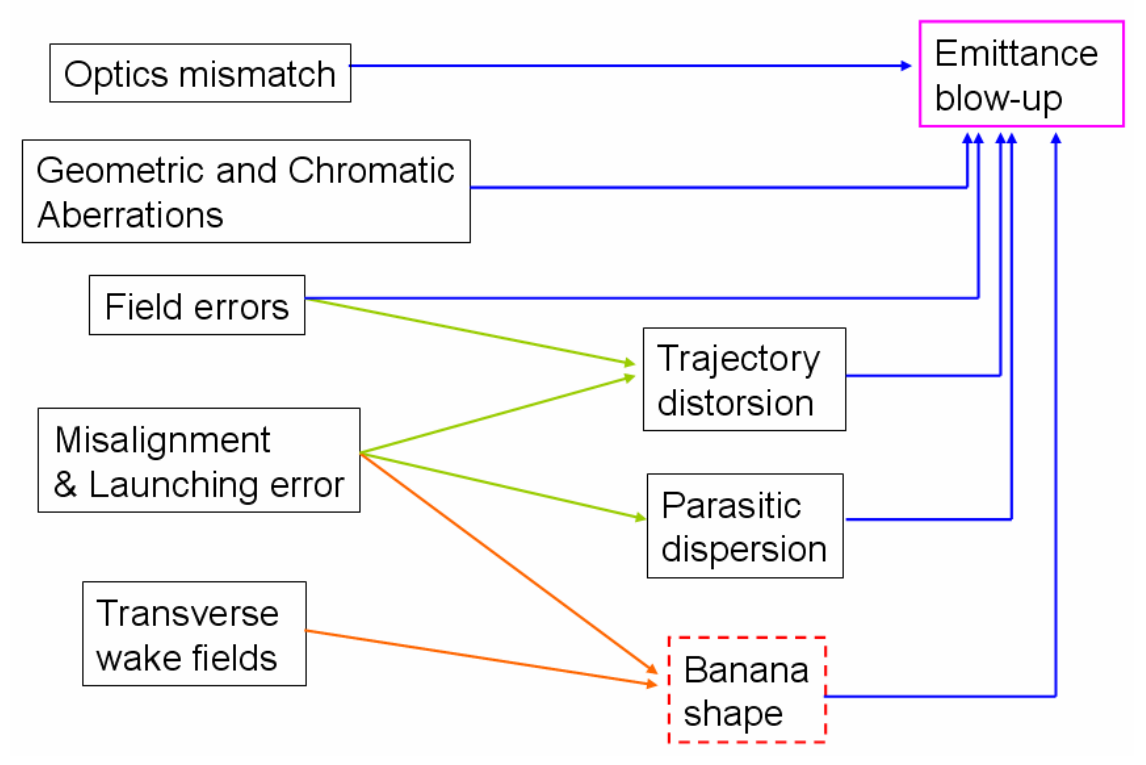

Figure 4.1 Sources of transverse emittance blow up for an electron beam traveling into a linac (CSR is ignored).

In this Chapter we discuss the sources of emittance blow up in the Fermi Linac, its analytical estimations and the simulation results. We anticipate that the Beam Break-Up (BBU) instability has been recognized as the main source of emittance dilution; related methods for the emittance preservation are also discussed. Special care has been taken in the treatment of the chromatic aberrations because of the large energy spread which occurs for the magnetic compression of the bunch length.

\subsection{Transverse dynamics without collective effects}

In this Section the emittance excitation due to the single particle dynamics effects is discussed. Contributions from the aberrations are evaluated and the effect of quantum fluctuations in the dispersive regions is estimated.

\subsubsection{Chromatic aberrations}

Emittance growth due to the chromatic aberration is the consequence of the chromatic dependence of the betatron phase advance, which leads to the emittance dilution by chromatic filamentation.

\subsubsection{Dipoles}

A quadrupolar and sextupolar field component in a dipole with horizontal dispersion $\eta_{\mathrm{x}}$ and horizontal beta function $\beta_{\mathrm{x}}$ will dilute the horizontal emittance through the generation of first and second order horizontal dispersion, respectively. Tolerances for the 
quadrupolar $\left(b_{1}\right)$, the sextupolar $\left(b_{2}\right)$ component at a radius $\mathrm{R}$ from the magnetic axis w.r.t. the main field component $\left(\mathrm{b}_{0}\right)$ are given by [30]:

$$
\begin{aligned}
& \left|\frac{b_{1}}{b_{0}}\right|<\frac{1}{\left|\theta_{b}\right|} \frac{R}{\eta_{x} \sigma_{\delta}} \sqrt{\frac{2 \varepsilon_{x 0}}{\beta_{x}} \frac{\Delta \varepsilon_{x}}{\varepsilon_{x 0}}} \\
& \left|\frac{b_{2}}{b_{0}}\right|<\frac{1}{\left|\theta_{b}\right|}\left(\frac{R}{\eta_{x} \sigma_{\delta}}\right)^{2} \sqrt{\frac{\varepsilon_{x 0}}{\beta_{x}} \frac{\Delta \varepsilon_{x}}{\varepsilon_{x 0}}}
\end{aligned}
$$

If we calculate the multipolar component at half gap $\mathrm{R}=20 \mathrm{~mm}$, then an emittance growth of less than $1 \%$ is guaranteed by (stronger constraint over all dipoles): $\left|b_{1} / b_{0}\right|<$ $0.6 \times 10^{-4}$ at BC1 and $\left|b_{1} / b_{0}\right|<1.5 \times 10^{-4}$ at BC2 as for the quadrupolar component; $\left|b_{2} / b_{0}\right|<$ $1.2 \times 10^{-4}$ at $\mathrm{BC} 1$ and $\left|\mathrm{b}_{2} / \mathrm{b}_{0}\right|<10 \times 10^{-4}$ at $\mathrm{BC} 2$ as for the sextupolar component (all results are relative to the maximum bending angle admitted, see Section $\mathrm{X}$ ). Because of the very small energy spread at the Spreader $\left(\sigma_{\delta}<0.1 \%\right)$, tolerances about the multipolar components in those dipoles are more relaxed.

\subsubsection{Quadrupoles}

The chromatic aberration in a quadrupole is a $2^{\text {nd }}$ order effect in particle coordinate; it depends on the integrated quadrupole strength $(k l)$, on the $\beta$-function at the quadrupole and on the relative energy spread of the particle $\left(\sigma_{\delta}\right)$ :

$$
\frac{\Delta \varepsilon}{\varepsilon} \cong \frac{1}{2} \beta^{2} \sigma_{\delta}^{2}(k l)^{2}
$$

The more critical point of the lattice causing chromatic aberration is expected to be in proximity of the first bunch compressor $(\mathrm{BC} 1)$, where the RMS correlated energy spread may reach a maximum value of about 3\%. (4.3) has been evaluated and summed over all the Linac sections, giving a total contribution to the emittance blow up of less than $6 \%$. Particle tracking (see, Figure 4.2) confirms the negligible emittance dilution. Tracking has been performed using the Elegant code [24], in which the quadrupoles have been treated as single-matrix elements including up to $3^{\text {rd }}$ order terms. 

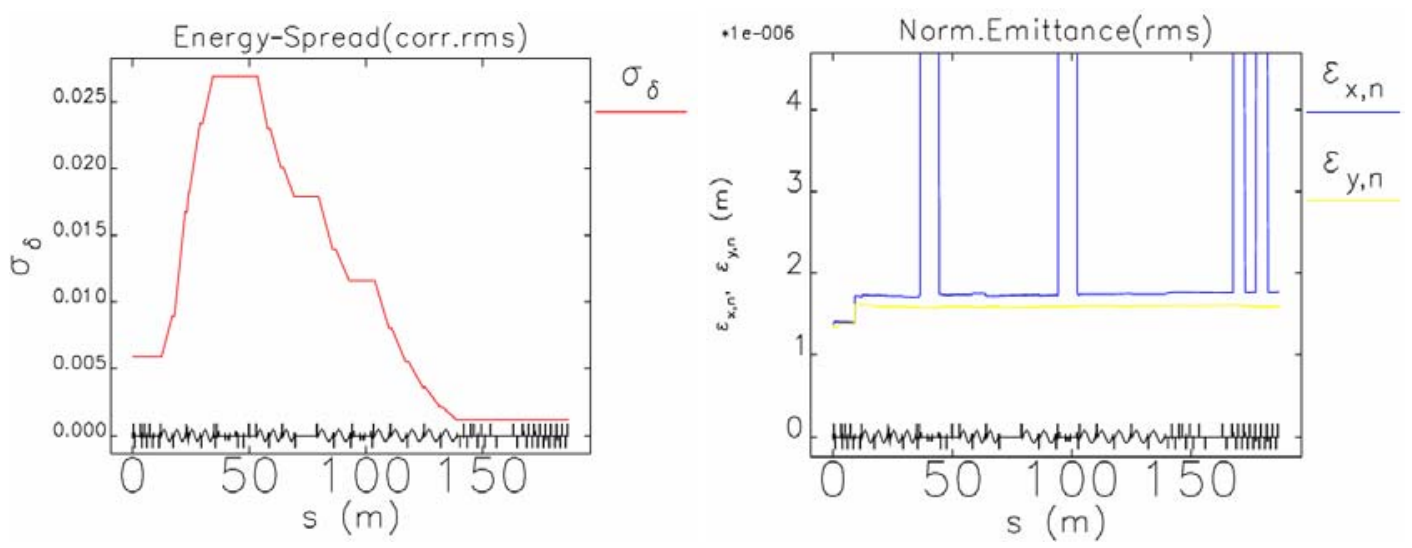

Figure 4.2 Evolution of the RMS relative energy spread (left) and of the normalized transverse emittance (right) along the Fermi Linac. The maximum value for the energy spread is in correspondence of $\mathrm{BC} 1$. The emittance includes the chromatic contribution, as shown by the large values it reaches in the dispersive regions of the lattice (BC1, BC2 and Spreader); nevertheless, it is preserved as the dispersion returns to the zero level (its first incremental step is an artificial effect implemented in the Elegant code for start-toend simulation purposes).

\subsubsection{Geometrical aberrations}

Geometrical aberrations describe the dependence of the focusing strength felt by a particle on its transverse position at $2^{\text {nd }}$ order. In absence of sextupoles in the Linac, the systematic sextupolar component of the dipole field has been recognized as the main source of geometrical aberration; any non-systematic octupolar component of the quadrupole field is assumed to be much less effective.

\subsubsection{Dipoles}

The emittance excitation by geometrical aberration can be estimated in the following way:

$$
\frac{\Delta \varepsilon}{\varepsilon} \cong \frac{1}{2}\left(k_{2} l\right)^{2} x_{0}^{4}\left(\beta+\alpha l+\frac{1}{4} \gamma l^{2}\right) \approx \frac{1}{2}\left(k_{2} l\right)^{2} \beta^{3} \varepsilon
$$

The sextupolar component is here defined like the ratio between the higher order field component $(n=3)$ and the main dipolar component at an arbitrary distance from the magnetic axis. If we assume a half gap of $20 \mathrm{~mm}$ for the dipole magnet, a maximum sextupolar component of $4 \times 10^{-4}$ at $20 \mathrm{~mm}$ leads to an emittance excitation of less than $1 \%$.

\subsubsection{Quantum fluctuations (ISR)}


High energy electrons passing through a curved beam transport system emit Incoherent Synchrotron Radiation (ISR) [31, 32, 33] and suffer from quantum excitation which is not compensated by damping (since there is no acceleration); in such case, the transverse emittance increases linearly with time [31]:

$$
\frac{d \varepsilon_{x}}{d s}=\frac{55}{48 \sqrt{3}} \frac{r_{e} \hbar c}{m c^{2}} \gamma^{5} \frac{H(s)}{\rho(s)^{3}}=4 \cdot 10^{-8} \cdot E^{5}[\mathrm{GeV}] \cdot I_{5}[\mathrm{~m}]
$$

where $\mathrm{I}_{5}$ is the fifth synchrotron integral and $\mathrm{H}(\mathrm{s})$ is the "curly- $\mathrm{H}$ " function depending on the horizontal dispersion $\eta_{\mathrm{x}}(\mathrm{s})$ and on the Twiss functions:

$$
H=\beta_{x} \eta_{x}^{\prime}+2 \alpha_{x} \eta_{x} \eta_{x}^{\prime}+\gamma_{x} \eta_{x}
$$

The emittance blow up is calculated from (4.5) integrating over the curved path; for a symmetric chicane of identical rectangular magnets we obtain [31]:

$$
\Delta \varepsilon_{x} \approx 8 \cdot 10^{-8} \cdot E^{5}[G e V] \cdot \frac{\theta^{5}}{l_{b}^{2}}\left[\left(L+l_{b}\right)+\left(\frac{\hat{\beta}+\stackrel{\vee}{\beta}}{3}\right)\right]
$$

(4.5) shows a strong energy dependence of the emittance blow up and therefore the effect becomes only significant for very high beam energies; thus, the largest effect is expected to be in $\mathrm{BC} 2$ at $600 \mathrm{MeV}$ and in the Spreader at $1.2 \mathrm{GeV}$. The evaluation of (4.7) for $\mathrm{BC} 1, \mathrm{BC} 2$ and the simulation result for the Spreader are summarized in Table 4.1. Notice that (4.4) and (4.7) are valid for a phase space ellipse perturbed by the radiation which is still matched to the Twiss ellipse of the transport line; this means that we are assuming $\Delta \varepsilon_{\mathrm{X}} / \varepsilon_{\mathrm{x}}<<1$.

Results in Table 4.1 demonstrate that the horizontal emittance excitation due to quantum fluctuations in the magnetic chicanes and in the Spreader is negligible. This also shows that a manipulation of the "curly- $\mathrm{H}$ " function in such regions in order to reduce the emittance blow up is not necessary.

Table 4.1 Over-estimated emittance blow up due to the ISR emitted in the dipoles of $\mathrm{BC} 1$, of $\mathrm{BC} 2$ and of the Spreader (calculated for the maximum bending angles admitted and for an unperturbed normalized emittance of $1.5 \mu \mathrm{m}$ ).

\begin{tabular}{lcc}
\hline & $\Delta \varepsilon_{\mathrm{x}}[\mu \mathbf{m}]$ & $\Delta \varepsilon_{\mathrm{x}} / \varepsilon_{\mathrm{x}}[\%]$ \\
\hline $\mathrm{BC} 1$ & $1.4 \times 10^{-8}$ & $4 \times 10^{-4}$ \\
\hline BC2 & $1.7 \times 10^{-6}$ & 0.1 \\
\hline SPREADER & $<2 \times 10^{-6}$ & $<0.3$ \\
\hline
\end{tabular}




\subsection{Trajectory distortion}

Alignment and field errors cause trajectory distortion (coherent motion of electrons) and emittance blow up (incoherent motion of electrons). The latter has a direct impact on the final beam quality, the former becomes an issue if complementing to the collective effect induced by the transverse wake fields (see, Section 4.3). In this Section the emittance dilution and the trajectory correction scheme are discussed. Results from the trajectory correction will be used in the next Section 4.3 for the treatment of the Beam Break-Up (BBU) instability.

Misalignment studies have been performed under the assumption of independent mechanical supports for each of the magnetic elements and accelerating structures. Ground motion and vibrations have been assumed to be much smaller than the static errors applied to the lattice.

\subsubsection{Misalignment and field errors}

Errors in the magnets field and in the elements alignment may lead to a trajectory distortion which has to be corrected by mean of a properly arranged scheme of steerers and Beam Position Monitors (BPMs). Such errors are also sources of mismatch between the beam phase space ellipse and the Twiss ellipse of the transport line. The chromatic dependence of the betatron phase advance may determine the chromatic filamentation of the phase space and it is responsible for the consequent emittance dilution. In the following, some criterions for the estimation of tolerances on the magnets field quality and their alignment are presented.

\subsubsection{Dipoles}

A roll angle $\phi_{\mathrm{b}}$ of the dipoles in may generate a residual vertical dispersion which in turn leads to a vertical emittance dilution. Tolerance for the dipole roll angle is given by [30]:

$$
\phi_{b}<\frac{1}{\theta_{b} \sigma_{\delta}} \sqrt{\frac{2 \varepsilon_{y 0}}{\beta_{y}} \frac{\Delta \varepsilon_{y}}{\varepsilon_{y 0}}}
$$

If we want $\frac{\Delta \varepsilon_{y}}{\varepsilon_{y 0}} \leq 1 \%$, then the $\quad$ stronger constraint comes from the maximum bending angle at $\mathrm{BC} 1\left(\theta_{\mathrm{b}}=0.085 \mathrm{rad}\right)$ in presence of the maximum relative energy spread $\left(\sigma_{\delta}=3 \%\right)$, giving $\phi_{\mathrm{b}} \leq 830 \mu \mathrm{m}$ (RMS value).

A dipole field error induces emittance blow up through dispersive effect according to [34]:

$$
\frac{\Delta \varepsilon}{\varepsilon}(s) \cong \frac{1}{2} N_{M} \beta^{2} \frac{\sigma_{e r}^{\prime 2}}{\sigma_{\beta}^{2}}
$$


where $\mathrm{N}_{\mathrm{M}}$ is the number of magnets affected by the error $\sigma_{e r}^{\prime}=\left(\frac{\Delta B \cdot l}{B \rho}\right)_{r m s}$. A relative dipole field error of $5 \times 10^{-5}$ contributes to an emittance blow up of less than $1 \%$.

\subsubsection{Quadrupoles}

A roll angle $\phi_{\mathrm{q}, 1}$ of a quadrupole in a dispersion free region induces geometrical coupling. The tolerance for the quadrupole roll angle is given by [30]:

$$
\phi_{q, 1}<\frac{1}{|k l|} \sqrt{\frac{2}{\beta_{x} \beta_{y}} \frac{\varepsilon_{y 0}}{\varepsilon_{x 0}} \frac{\Delta \varepsilon_{y}}{\varepsilon_{y 0}}}
$$

If we want $\frac{\Delta \varepsilon_{y}}{\varepsilon_{y 0}} \leq 1 \%$, we get the relaxed constraint of $\phi_{\mathrm{q}, 1} \leq 20 \mathrm{mrad}$ (RMS value) for an average focusing length $\mathrm{f}=1 / \mathrm{kl}=5 \mathrm{~m}$ and average betas of $20 \mathrm{~m}$.

In presence of dispersion the skew field component leads to a residual vertical dispersion and therefore to the vertical emittance dilution. In this case, the tolerance for the quadrupole roll angle becomes [30]:

$$
\phi_{q, 2}<\frac{1}{|k l|} \frac{1}{\eta_{x} \sigma_{\delta}} \sqrt{\frac{2 \varepsilon_{y 0}}{\beta_{y}} \frac{\Delta \varepsilon_{y}}{\varepsilon_{y 0}}}
$$

The condition $\frac{\Delta \varepsilon_{y}}{\varepsilon_{y 0}} \leq 1 \%$ is satisfied by $\phi_{\mathrm{q}, 2} \leq 10 \mathrm{mrad}$ (RMS value) for an uncorrected horizontal dispersion of $1 \mathrm{~cm}$ and an RMS relative energy spread of 3\% (conservative values).

A lateral misalignment of the quadrupole also generates residual dispersion in both planes and to avoid a relevant emittance growth the transverse alignment of the magnet has to be [30]:

$$
\Delta x, \Delta y<\frac{1}{|k l|} \frac{1}{\sigma_{\delta}} \sqrt{\frac{2 \varepsilon_{0}}{\beta} \frac{\Delta \varepsilon}{\varepsilon_{0}}}
$$

If we want again $\frac{\Delta \varepsilon}{\varepsilon_{0}} \leq 1 \%$, we obtain the important constraint $\Delta \mathrm{x}, \Delta \mathrm{y} \leq 110 \mu \mathrm{m}$ (RMS value) for a conservative value of the energy spread $\sigma_{\delta}=3 \%$.

Finally, field errors in both the normal and skew component of the quadrupole gradient generate emittance dilution according to [34]:

$$
\frac{\Delta \varepsilon}{\varepsilon} \cong \frac{1}{2} N_{M} \beta^{2} k^{2} l^{2}\left(\sigma_{\alpha}^{2}+\sigma_{k}^{2}\right)
$$

where $\alpha=k \phi_{\mathrm{q}, 1}$ and $\mathrm{N}_{\mathrm{M}}$ is the number of quadrupoles in the line. An RMS gradient error $\sigma_{\mathrm{k}}=0.1 \%$ and an RMS roll angle $\phi_{\mathrm{q}, 1}=300 \mu \mathrm{m}$ guarantee an emittance blow up of the order of $0.1 \%$. 


\subsubsection{Launching error}

An optical mismatch of the beam phase space ellipse incoming from the Injector with the Twiss ellipse defined by the Linac optics may cause emittance blow up through chromatic filamentation. In the pessimistic ipothesis of complete filamentation, a $\beta$ mismatch and a $\eta$-mismatch at the beginning lead to, respectively:

$$
\begin{gathered}
\alpha_{i}=0 \Rightarrow \frac{\Delta \varepsilon}{\varepsilon} \cong \frac{1}{2}\left(\frac{\Delta \beta}{\beta}\right)^{2} \\
\frac{\Delta \varepsilon}{\varepsilon_{\beta}}(s) \cong \frac{1}{2} \sigma_{\delta}^{2}\left(\frac{\widetilde{\eta}^{2}+\left(\alpha \widetilde{\eta}+\beta \widetilde{\eta}^{\prime}\right)^{2}}{2 \varepsilon_{0} \beta}\right)
\end{gathered}
$$

A $\beta$-mismatch of $5 \%$ may cause an emittance blow up of $13 \%$, while a residual dispersion $\widetilde{\eta}=5 \cdot 10^{-3} \mathrm{~m}$ with divergence $\widetilde{\eta}^{\prime}=5 \cdot 10^{-4}$ may generate a blow up until $15 \%$. At the injection into the Linac, the residual chromatic beam size should not exceed the geometrical one; hence in general it has to be $\widetilde{\eta} \ll \sigma_{\beta, 0} / \sigma_{\delta, 0} \approx 10 \mathrm{~cm}$.

In the simulations, the beam incoming from the Injector already includes an optical mismatch of few percents in the projected Twiss functions; moreover, their distribution in the beam slices is not uniform due to the effect of the space charge dynamics at low energy.

The beam centroid is assumed to be affected by a transverse offset which defines the launching error for the beam traveling into the Linac. A lateral displacement of $100 \mu \mathrm{m}$ and an angular divergence of $100 \mu \mathrm{rad}$ have been applied to the $\mathrm{x}$ and $\mathrm{y}$ plane. Actually, these errors do not match with the phase space described by a given normalized emittance of about $1 \mathrm{~mm} \mathrm{mrad}$ and $\beta$-functions of $19 \mathrm{~m}$ at their waist; thus, such initial conditions over-estimate the realistic launching error and make the simulations a conservative scenario.

\subsubsection{Trajectory correction}

A satisfactory trajectory correction can be obtained in the $120 \mathrm{~m}$ long Fermi Linac by using 40 pairs of correctors and 40 BPMs, active on both the transverse planes. Each drift between two consecutive accelerating structures includes one BPM and one steerer, separated by a quadrupole magnet. This scheme allows for providing two different methods of correction:

(i) one-to-one trajectory correction, that is each steerer corrects the beam position at the BPM located at the end of the downstream accelerating structure; 
(ii) global trajectory correction that is all the steerers and all the BPMs are involved in the minimization of the divergence and of the position of the beam centroid along the whole Linac.

In the optical matching sections (i.e., at the conjunction of the Injector with the Linac and of the Linac with the Undulators Line, in the proximity of $\mathrm{BC} 1$ and of $\mathrm{BC} 2$ ) the distance between two consecutive steerers (active on the same plane) should not extend for more than 5 meters (over which an angular kick error of $0.1 \mathrm{mrad}$ may cause a beam deflection of $500 \mu \mathrm{m})$.

The order of magnitude of the angular kicks provided by the field and alignment errors have been estimated; they result being much smaller than the average kick which should be provided by the steerers for the trajectory correction:

$$
\left.\begin{array}{ll}
\text { Dipole field error: } & \theta_{d}=\frac{\Delta\left(\int B d l\right)}{B \rho} \leq 100 \mu \mathrm{rad} \\
\text { Gradient error: } & \theta_{q}=\Delta K \cdot L \cdot x_{\beta} \leq 1 \mu \mathrm{rad} \\
\text { Misalign. Quads: } & \theta_{q}=K \cdot L \cdot \Delta x_{\text {misal }} \leq 60 \mu \mathrm{rad}
\end{array}\right\}<<\text { kick for correction } \approx \mathrm{mrad}
$$

In the simulations, BPMs have been misaligned assuming a lateral displacement Gaussian distributed with a standard deviation of $150 \mu \mathrm{m}$ and cutoff at 3- $\sigma$. BPMs resolution has been supposed to be affected by reading noise Gaussian distributed with a standard deviation of $30 \mu \mathrm{m}$ and a cutoff at $2-\sigma$ (thus, the uncertainty in the BPM readout is between 0 and $60 \mu \mathrm{m}$ ). This set up forces the trajectory correction to an average kick per steerer of $1.5 \mathrm{mrad}$. Figure 4.3 shows that the RMS off axis-trajectory is within $700 \mu \mathrm{m}$ peak-to-peak in both planes.

Notice that particle tracking has been performed simulating a realistic operation of the machine: the beam centroid is read by the code only at the BPMs locations and the BBU instability has been included in the trajectory correction (see, Section 4.3.2) since it modifies the transverse distribution of the particles position and finally the computation of the centroid position at the BPMs. Figure 4.3 shows an ensemble of trajectories, including field errors, elements misalignment and trajectory correction; it is based on the pessimistic tolerance errors budget reported in Tables 4.2, 4.3 and 4.4.

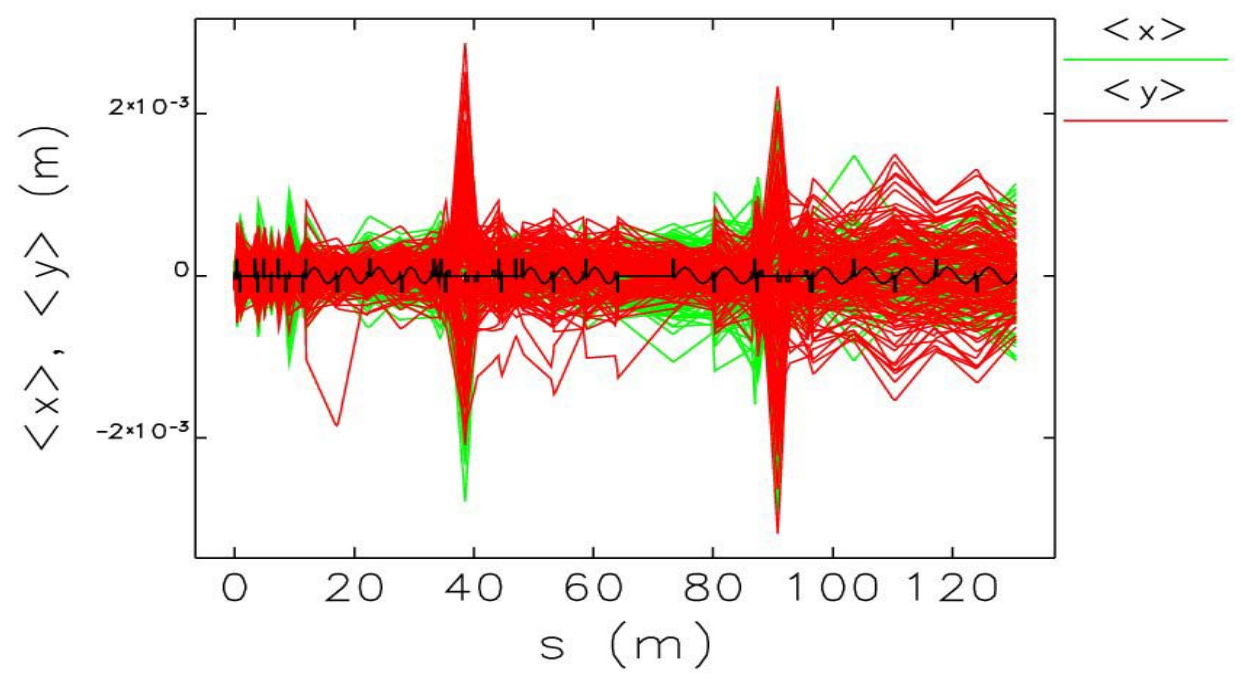


Figure 4.3 Ensemble of 120 trajectories along the Fermi Linac after correction (Elegant result). It includes field errors and elements misalignment reported in the pessimistic tolerance errors budget in Tables 4.2, 4.3 and 4.4. The large excursion in the chicanes is shown w.r.t the straight path.

Table 4.2 Elements misalignment (RMS values).

\begin{tabular}{lccc}
\hline & $\Delta \mathbf{x}, \Delta \mathbf{y}[\mu \mathbf{m}]$ & $\Delta \mathbf{z}[\mu \mathbf{m}]$ & $\Delta \theta[\mu \mathbf{r a d}]$ \\
\hline Dipole & - & - & 300 \\
\hline Quadrupole & 150 & 200 & 300 \\
\hline BPM $(30 \mu \mathrm{m}$ RMS resolut. $)$ & 150 & 200 & - \\
\hline Acc. Structure & 300 & - & - \\
\hline
\end{tabular}

Table 4.3 Field quality (RMS values).

\begin{tabular}{lc}
\hline & $\begin{array}{c}\text { Main field component } \\
{[\% \mathbf{\%}]}\end{array}$ \\
\hline Dipole & $\Delta \mathrm{B} / \mathrm{B}=0.01$ \\
\hline Quadrupole & $\Delta \mathrm{K} 1 / \mathrm{K} 1=0.1$ \\
\hline
\end{tabular}

Table 4.4 Launching error

\begin{tabular}{lcc}
\hline & Nominal Set & Jitter [p-t-p] \\
\hline$|\Delta \mathrm{x}|,|\Delta \mathrm{y}|$ & $100 \mu \mathrm{m}$ & $<100 \mu \mathrm{m}$ \\
\hline$\left|\Delta \mathrm{x}^{\prime}\right|,\left|\Delta \mathrm{y}^{\prime}\right|$ & $100 \mu \mathrm{rad}$ & - \\
\hline
\end{tabular}

\subsection{Transverse dynamics with collective effects}

This Section treats the transverse wake function characterizing the three types of accelerating structure which constitute the Fermi Linac [12, 35]. The short-range transverse wake field excited by the electron beam passing off-axis in the structures has been computed in a semi-analytical way (Section 4.3.1) and then used for the Elegant particle tracking. The effect of the wake field on the electron bunch itself has been estimated analytically and compared with simulations (Section 4.3.2). The angular kick provided to the beam produces a transverse deviation of the bunch tail w.r.t the head (the so-called "banana" shape); the bunch tail continues performing transverse coherent oscillations as the beam travels along the Linac. The resulting Beam Break-Up (BBU) instability has been recognized as the main source of emittance dilution in the Linac. For 
this reason, possible solutions for the compensation of the induced banana shape have been considered; local and non-local methods of trajectory correction have been chosen and simulated with success.

\subsubsection{Transverse wake fields}

The knowledge of the short-range wakefields in the accelerating sections is needed to predict the beam quality in term of single bunch energy spread and emittance. S0A and S0B and CERN sections $[12,35]$ are traveling wave (TW) structures which work with $2 / 3 \pi$ mode and cells on axis coupled. Figure 4.4 shows a schematic geometry of the TW accelerating structure. The Elettra sections $[12,35]$ are backward traveling wave (BTW) structures coupled magnetically which work with $3 / 4 \pi$ mode. Figure 4.5 shows a schematic view of the BTW accelerating structures. The cells in the BTW structures are magnetically coupled, thus demanding a small iris radius compared to the custom traveling wave structures; this cause stronger longitudinal and transverse wakefields than in the TW structures. For the parameters and basic dimensions of all the accelerating structures we send to Table 2.1 in Section 2.2.1.

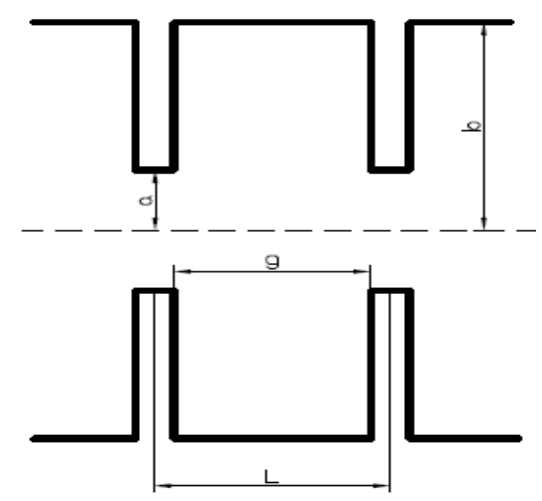

Figure 4.4 Schematic geometry of the TW accelerating structure (S0A, S0B and CERN sections).

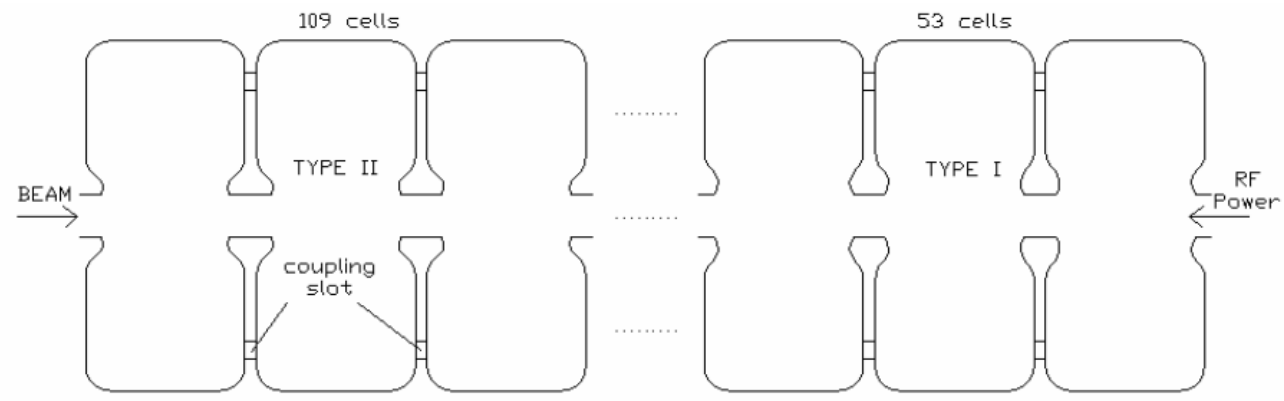

Figure 4.5 Schematic view of the BTW accelerating section geometry (S1-S7). 
As suggested in Refs. [36, 37], an analytical approximation of the wake function for the TW structure (S0A, S0B and CERN sections) may be used for the transverse case:

$$
w_{\perp}^{1}(s)=\frac{4 Z_{0} c}{\pi a^{4}} s_{1}\left[1-\left(1+\sqrt{\frac{s}{s_{1}}}\right) e^{-\sqrt{\frac{s}{s_{1}}}}\right][\mathrm{V} / \mathrm{pC} / \mathrm{m} / \mathrm{m}]
$$

with:

$$
s_{1}=0.169 \cdot \frac{a^{1.79} g^{0.38}}{L^{1.17}}[\mathrm{~m}]
$$

where $a$ is the iris radius, $g$ is the cell gap, $L$ is the cell period and $Z_{0}$ is the resistance of free space. This analytical model has been calculated for geometrical parameters in the region:

$$
0.34 \leq \frac{a}{L} \leq 0.69,0.54 \leq \frac{g}{L} \leq 0.89 \text { and } 0 \leq \frac{s}{L} \leq 0.16
$$

\subsubsection{Wake function for SOA and SOB structures}

With the geometrical parameters in Table 2.1, Section 2.2.1 we obtain for the transverse wake function up to $5 \mathrm{~mm}$ ( $\mathrm{s}$ in meters):

$$
w_{\perp}^{1}(s)=9623 \cdot\left[1-\left(1+\sqrt{\frac{s}{0.60 \cdot 10^{-3}}}\right) \cdot e^{-\sqrt{s / 0.6010^{-3}}}\right]\left[\frac{\mathrm{V}}{p C \cdot m \cdot m}\right]
$$

We can observe that in this case the geometrical parameters are not in the validity range: $a / L=0.292$ and $g / L=0.91$. For this reason it has been decided to check the models for the description of the wake functions with numerical simulations.

\subsubsection{Wake function for $C$ structures}

As in the previous case, using the parameters in Table 2.1, Section 2.2.1 we obtain for the transverse wake function up to $5 \mathrm{~mm}$ ( $\mathrm{s}$ in meters):

$$
w_{\perp}^{1}(s)=7524 \cdot\left[1-\left(1+\sqrt{\frac{s}{0.70 \cdot 10^{-3}}}\right) \cdot e^{-\sqrt{\% / 0.7010^{-3}}}\right]\left[\frac{\mathrm{V}}{p C \cdot m \cdot m}\right]
$$

In this case the parameters $a / L=0.323$ is not in the validity range; thus the model has been checked by numerical simulations. 


\subsubsection{Wake function for BTW structures}

For this kind of accelerating structure we have studied the short-range longitudinal and transverse wake potentials using a time domain code and the results are reported in Ref. 36]. From the fit of the numerical wake potentials we have obtained analytical expressions approximating the longitudinal and transverse wake functions. As for the analytical model, we have chosen a combination of periodic and one-cell structure solutions. The transverse wake function up to $2 \mathrm{~mm}$ is ( $\mathrm{s}$ in meters):

$$
w_{\perp}^{1}(s)=27984 \cdot\left[1-\left(1+\sqrt{\frac{s}{0.12 \cdot 10^{-3}}}\right) \cdot e^{-\sqrt{s / 2.12 \cdot 10^{-3}}}\right]+13992 \cdot \sqrt{\mathrm{s}}\left[\frac{\mathrm{V}}{p C \cdot m \cdot m}\right]
$$

Figure 4.6 shows the transverse wake functions for all the accelerating structures involved in the Fermi Linac. We can see that S0A, S0B and CERN sections are characterized by relatively weak wake fields compared with the S-sections, where the wake fields are largely more effective.

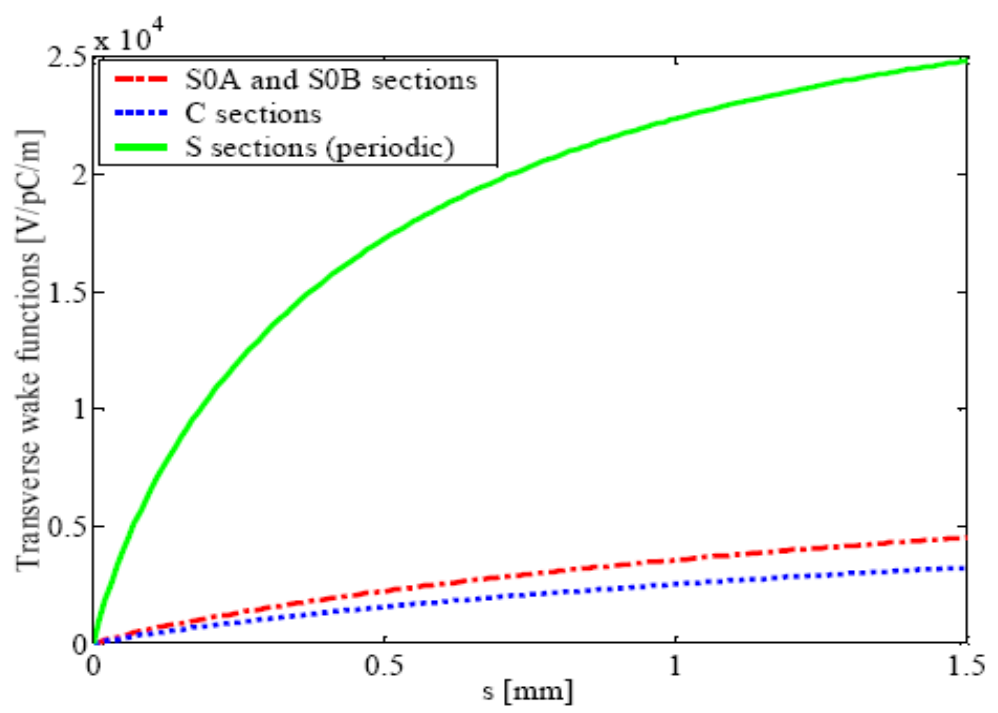

Figure 4.6 Transverse wake functions for the accelerating structures in the FERMI linac.

\subsubsection{Beam break-up instability}

Beam Break-Up instability (BBU) has been recognized as the main source of emittance dilution in the Fermi Linac. An electron beam traveling off-axis in an accelerating structure excites the short-range transverse wake field which in turn gives a kick distributed along the bunch length. The bunch tail starts oscillating w.r.t. to the head axis, so forming in the t-x and t-y plane the so-called "banana" shape. Lasting of such oscillations along the Linac and their amplification may cause the beam break up that is the total conversion of the bunch time duration into the transverse dimension. Thus, the 
projected geometric emittance results largely increased and the time structure of the beam is lost.

The relevant wake fields in the S1-S7 Linac sections (see, Figure 4.6, Section 4.2) make this part of the layout particularly sensitive to the BBU instability. Possible solutions have been considered, referring to the operation of well-known linear colliders. So far, the most used techniques in the suppression of the BBU instability are:

- High Order Mode (HOM) dampers for long-range wake fields [38, 39];

- Balakin Novohatsky Smirnov (BNS) damping [40, 41, 42, 43];

- Local trajectory bumps [44].

The HOM dampers are not useful for the treatment of the short-range wake fields effective in the Fermi Linac.

The BNS damping foresees an energy-position correlation in the longitudinal phase space with a sign opposite to that provided by the dynamics of compression (see, Section 2). Even if some of the S1-S7 accelerating structures could be set off-crest $(\Delta \phi>0)$ in order to create such a correlation, the constraints from the FEL process on the final properties of the beam like the average energy and mostly the linear energy chirp and the small energy spread make the BNS energy balance unfeasible for the present Fermi Linac. The BNS damping option would imply the addition of accelerating structures to the present layout.

The option of local trajectory bumps is feasible in the present Fermi Linac. This method consists in an empirical search of a "golden" trajectory which makes the kicks provided from the wake fields to the bunch compensating each other. In this way the final induced banana shape can be cancelled. The goal of this technique is the reduction of the head-tail deviation within the nominal RMS beam size.

\subsubsection{Theoretical model}

In this section we present an analytical study based on a continuous model which describes the transverse motion of a single bunch in presence of transverse wake fields; it is contained in Ref. [45] and presented here for reader convenience. Such a study allows predicting the emittance growth under the combined influence of the short-range transverse wakefields, injection offset, initial emittance and misaligned accelerating sections. We also report a comparison between analytical and numerical (tracking code) results.

The main parameters for the last part of the Fermi Linac, called L4 [46], are listed in Table 4.5. The effects of the wake fields relative to two bunch lengths with uniform current distribution are compared, that is $200 \mu \mathrm{m}$ full width (FW) for the Short bunch and $500 \mu \mathrm{m}$ full width (FW) for the Medium bunch (see, Section 1).

Table 4.5 Nominal parameters for L4.

\begin{tabular}{|l|c|c|c|}
\hline Total initial energy & $\gamma(0)$ & 1174 & \\
Total final energy & $\gamma(1)$ & 2348 & \\
L4 total length & $L$ & 30.375 & \\
\end{tabular}




\begin{tabular}{|l|c|c|c|} 
Peak current & $I_{\max }$ & 800 & $\mathrm{~m}$ \\
Short bunch FW length & $l_{b}$ & 200 & $\mathrm{~A}$ \\
Medium bunch FW length & $l_{b}$ & 500 & $\mu \mathrm{m}$ \\
& & & $\mu \mathrm{m}$ \\
\hline
\end{tabular}

As indicated in Refs. [47, 48], in a continuous approximation the transverse motion of the relativistic beam in a misaligned accelerator under the influence of the short-range transverse wake fields can be modeled by:

$$
\frac{1}{\gamma(\sigma)} \frac{\partial}{\partial \sigma}\left[\gamma(\sigma) \frac{\partial}{\partial \sigma} x(\sigma, \zeta)\right]+\kappa(\sigma)^{2} x(\sigma, \zeta)=\varepsilon(\sigma) \int_{-\infty}^{\zeta} w_{n}\left(\zeta-\zeta_{1}\right) F\left(\zeta_{1}\right)\left[x\left(\sigma, \zeta_{1}\right)-d_{c}(\sigma)\right] d \zeta_{1}
$$

where $\gamma(\sigma)$ is the energy parameter along the linac; $\sigma=\mathrm{s} / \mathrm{L}$ is the distance from entrance of the linac normalized to $\mathrm{L} ; \zeta=\mathrm{z} / \mathrm{l}_{\mathrm{b}}$ is the normalized coordinate measured after the arrival of the head of the beam at location $\sigma ; \mathrm{F}(\zeta)=\mathrm{I}(\zeta) / \mathrm{I}_{\max }$ is the instantaneous current divided by the maximum current of the uniform current distribution; $\kappa=\mathrm{k} / \mathrm{L}$ is the focusing wave number $\mathrm{k}$ normalized to $\mathrm{L} ; \mathrm{w}_{\mathrm{n}}(\zeta)$ is the normalized transverse wake function; $\mathrm{d}_{\mathrm{c}}(\sigma)$ is the lateral displacement of the accelerating sections as a function of location along the linac; $\varepsilon(\sigma)=\left(\varepsilon_{\mathrm{r}} \gamma(0) / \gamma(\sigma)\right)$ is the dimensionless "coupling strength" between the beam and the transverse wakes; $\varepsilon_{\mathrm{r}}$ for the short-range wakes is defined by:

$$
\varepsilon_{r}=\frac{4 \pi \varepsilon_{0} W_{0} I_{\max } l_{b} L^{2}}{\gamma(0) I_{A}}
$$

where $\mathrm{I}_{\mathrm{A}} \cong 17000 \mathrm{~A}, \varepsilon_{0}$ is the dielectric constant of the vacuum and $\mathrm{W}_{0}$ is the wake amplitude. Refs. [48,49] give the general solution of the equation of motion (4.23) for an accelerated beam:

where:

$$
\begin{aligned}
x(\sigma, \zeta) & =\frac{1}{\sqrt[4]{\psi(\sigma)}} \sum_{n=0}^{\infty} \varepsilon_{r}^{n}\left\{x_{0} h_{n}(\zeta) j_{n}\left(\kappa_{r}, \varsigma\right)\right. \\
& \left.+\left[x_{0}^{\prime} g_{n}(\zeta)+x_{0} \frac{G}{4} h_{n}(\zeta)\right] i_{n}\left(\kappa_{r}, \varsigma\right)\right\} \\
- & \frac{1}{\sqrt[4]{\psi(\sigma)}} \sum_{n=0}^{\infty} \varepsilon_{r}^{n+1} f_{n+1}(\zeta) i_{n}\left(\kappa_{r}, \varsigma\right) * \delta_{c}(\varsigma) \\
\zeta & =\frac{2 \sigma}{\sqrt{\psi(\sigma)}+1}
\end{aligned}
$$

is a new variable for the longitudinal beam location along the linac.

In (4.25) $\mathrm{x}_{0}$ and $\mathrm{x}_{0}$ ' are the lateral displacement and the angular divergence, respectively, of the bunch at the entrance of the linac; they are time independent; $\psi(\sigma)=\gamma(\sigma) / \gamma(0)$ with $\gamma(\sigma)=1+\mathrm{G} \sigma$ and $\mathrm{G}$ the acceleration gradient; $\delta_{\mathrm{c}}=\psi^{1 / 4} \mathrm{~d}_{\mathrm{c}} ; \mathrm{i}_{\mathrm{n}}\left(\kappa_{\mathrm{r}} ; \varsigma\right)$ and $\mathrm{j}_{\mathrm{n}}\left(\kappa_{\mathrm{r}} ; \varsigma\right)$ are defined in ref. [48] in terms of Bessel functions and contain only powers and circular 
functions; $\mathrm{i}_{\mathrm{n}}\left(\kappa_{\mathrm{r}} ; \varsigma\right)^{*} \delta_{\mathrm{c}}$ is the convolution of $\mathrm{i}_{\mathrm{n}}$ and $\delta_{\mathrm{c}}$. With the assumption $\mathrm{x}_{0}$ and $\mathrm{x}_{0}{ }^{\prime}$ time independent, it is $\mathrm{f}_{\mathrm{n}}(\zeta)=\mathrm{g}_{\mathrm{n}}(\zeta)=\mathrm{h}_{\mathrm{n}}(\zeta)$ and they are defined by the recursion relations in Ref [48]. An analytical expression approximating the transverse wake function for BTW sections has been obtained in Section 4.3.1 in units of $\mathrm{V} / \mathrm{C} / \mathrm{m}^{2}$; notice that it depends on the total bunch length $1_{b}$. Table 4.6 reports the values for the Short and the Medium bunch cases, together with the dimensionless parameter $\varepsilon_{\mathrm{r}}$.

Table 4.6 Coupling strength $\varepsilon_{\mathrm{r}}$ in (4.24) as function of the parameters of the Short and Medium bunches and their related wake potential amplitudes.

\begin{tabular}{|c|c|c|c|}
\hline $\boldsymbol{l}_{\boldsymbol{b}}$ & $\boldsymbol{W}_{\boldsymbol{0}}$ & $\zeta_{\boldsymbol{l}}$ & $\mathcal{E}_{\boldsymbol{r}}$ \\
\hline$[\mu \mathrm{m}]$ & {$\left[\mathrm{V} / \mathrm{C} / \mathrm{m}^{2}\right]$} & & \\
\hline 200 & $1.048 \cdot 10^{16}$ & 0.600 & 8.620 \\
500 & $1.713 \cdot 10^{16}$ & 0.240 & 35.241 \\
\hline
\end{tabular}

Figure 4.7 shows on the left the normalized wake functions for the Short and Medium bunch cases together with their linear approximation. On the right, the functions $\mathrm{f}_{\mathrm{n}}(\zeta)$, $\mathrm{g}_{\mathrm{n}}(\zeta)$, and $\mathrm{h}_{\mathrm{n}}(\zeta)$ up to $\mathrm{n}=2$ (terms of higher order are negligible and the sum in (4.25) converges very fast). Using linear wakes instead of wake functions, the relative errors in the general solution (4.25) is about $20 \%$.
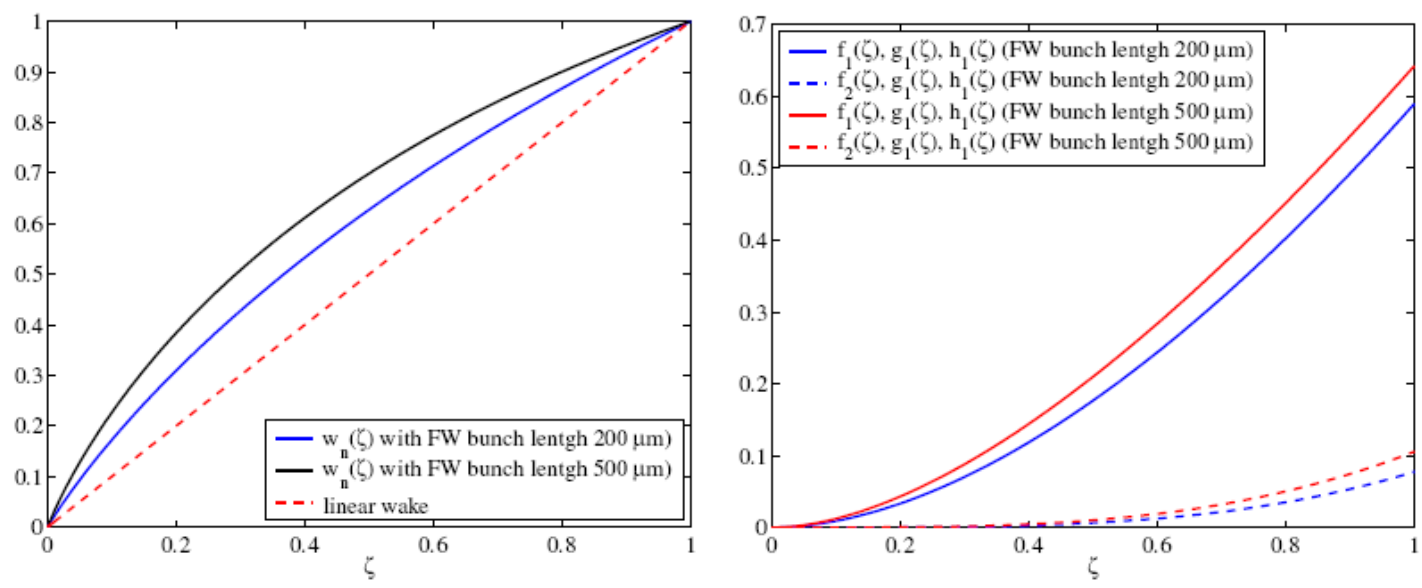

Figure 4.7: left, comparison between wake functions (Medium bunch: black line, Short bunch: blue line) and their linear approximation $\mathrm{w}(\zeta)=\zeta$ (red dashed line); right, functions $\mathrm{f}_{\mathrm{n}}(\zeta), \mathrm{g}_{\mathrm{n}}(\zeta)$, and $\mathrm{h}_{\mathrm{n}}(\zeta)$ up to the $2^{\text {nd }}$ order for the Medium and Short bunches.

\subsection{Effect of an initial lateral displacement}

If we consider the effects of an initial lateral offset of the beam in an aligned accelerator, we may assume for the general solution (4.25) $\mathrm{x}_{0}{ }^{\prime}=0$ and $\mathrm{d}_{c}(\sigma)=0$. Figure 4.8 shows the emittance growth as a function of position along the linac for the Short (a) and the Medium (b) bunch case. We have used two focusing wave numbers which cover approximately the range of the optics in L4. We can see that between the Short and the Medium case there is a factor 10 in the emittance growth. 


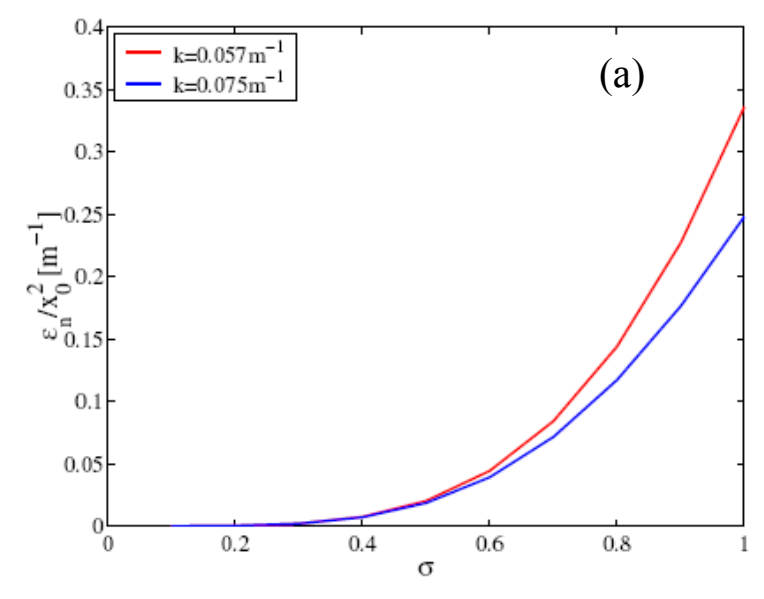

(a)

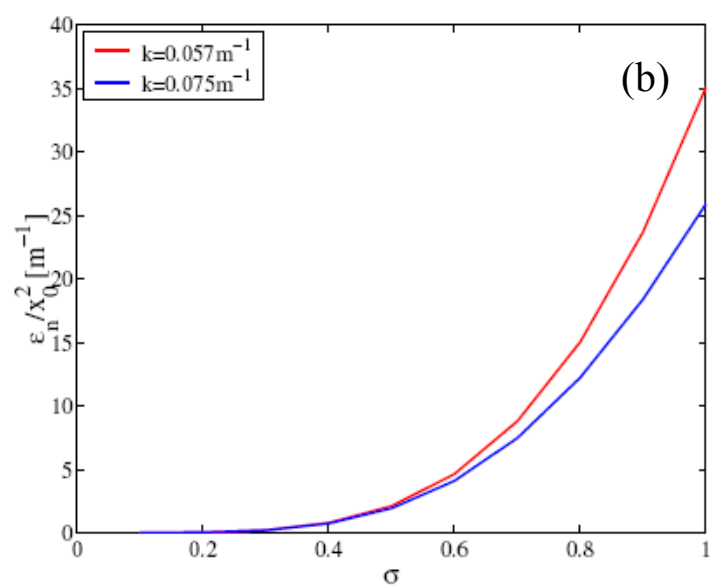

(b)

Figure 4.8: Normalized emittance growth along L4 in the Short (a) and Medium (b) bunch case.

In order to confirm the analytical results, they have been compared to the results from the Elegant tracking code. Figure 4.9 shows the lateral displacement (a) and the angular divergence (b) as a function of the position inside the bunch at L4 end for an initial offset of $200 \mu \mathrm{m}$. We can observe an excellent agreement between the analytical and the numerical results.

We have repeated the comparison for different initial offsets and the results are listed in Table 4.7. The comparisons have been performed even for the Medium bunch case, confirming once more the good agreement (not shown here). There are two discrepancies between the tracking and the analytical solution: (i) in Elegant we have foreseen a normalized focusing strength that does not change with the linac energy, while the analytical case foresees a focusing strength that decreases with the linac energy, i.e. $\mathrm{k}(\sigma)$ $=\mathrm{k}_{\mathrm{r}} / \sqrt{ } \psi(\sigma)$. For this reason we need small corrections on the focusing strength in the analytical model w.r.t. the tracking; (ii) we have found slight discrepancies in the centroid offset at the linac end, even if it has not influence on the calculation of the normalized projected emittance. 


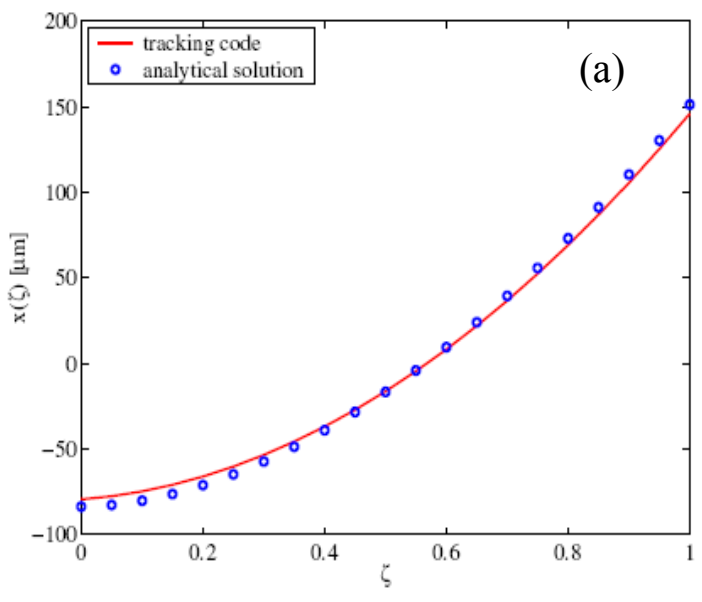

(a)

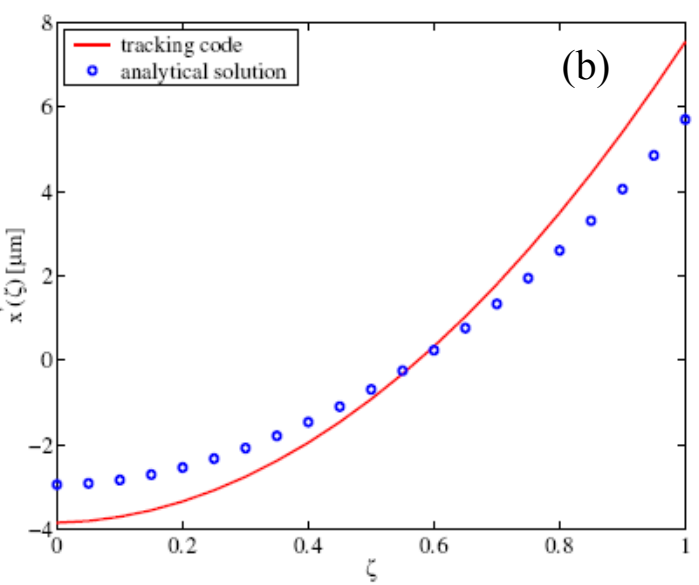

(b)

Figure 4.9: Comparison between analytical (blue circles) and tracking (red line) results for the lateral displacement (a) and angular divergence (b) at the L4 end due to an initial offset of $200 \mu \mathrm{m}$ in the Short bunch case.

Table 4.7 Comparison between analytical solution and ELEGANT tracking with FW bunch length of $200 \mu \mathrm{m}$ and different initial offset $\mathrm{X}_{0}$.

\begin{tabular}{|c|c|c|}
\hline & ELEGANT & Analytical \\
\hline $\mathrm{X}_{0}$ & $\varepsilon_{n, r m s}$ & $\varepsilon_{n, r m s}$ \\
\hline$[\mu \mathrm{m}]$ & {$[\mu \mathrm{rad}]$} & {$[\mu \mathrm{rad}]$} \\
\hline 50 & $0.08 \cdot 10^{-2}$ & $0.08 \cdot 10^{-2}$ \\
100 & $0.33 \cdot 10^{-2}$ & $0.34 \cdot 10^{-2}$ \\
150 & $0.75 \cdot 10^{-2}$ & $0.76 \cdot 10^{-2}$ \\
200 & $1.32 \cdot 10^{-2}$ & $1.34 \cdot 10^{-2}$ \\
250 & $2.07 \cdot 10^{-2}$ & $2.10 \cdot 10^{-2}$ \\
300 & $2.97 \cdot 10^{-2}$ & $3.02 \cdot 10^{-2}$ \\
500 & $8.24 \cdot 10^{-2}$ & $8.40 \cdot 10^{-2}$ \\
\hline
\end{tabular}

\subsection{Effect of misaligned BTW accelerating structures}

Here we want to consider the effects of the misaligned BTW accelerating structures with null lateral offset and null angular divergence of the beam at the entrance of the linac. We assume in the general solution (4.25) $\mathrm{x}_{0}=0$ and $\mathrm{x}_{0}{ }^{\prime}=0$. The specific case where the accelerating sections are all misaligned by the same amount $\mathrm{d}_{\mathrm{c}}$ is here considered. Figure 4.10 shows the normalized lateral displacements as a function of position inside the bunch at linac end for the Short and the Medium bunch case. We can see that the displacement of the bunch tail at linac end increases about by a factor 5 from the Short to the Medium bunch. Table 4.8 lists the normalized emittance growth for different misalignments $\mathrm{d}_{\mathrm{c}}$ of the BTW accelerating sections, for both the bunch length considered. In the Short bunch case the emittance growth is limited, while for the Medium bunch the 
emittance growth becomes important if the misalignment of the cavities is more than 100 $\mu \mathrm{m}$.

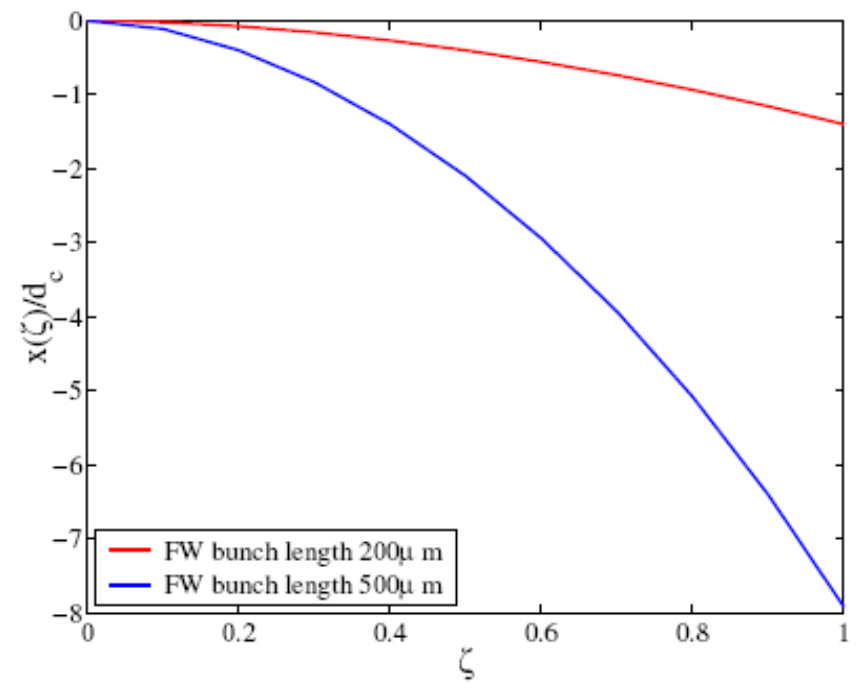

Figure 4.10: Normalized lateral displacements as a function of the position inside the bunch at the L4 end in the Short (red line) and Medium (blue line) bunch case.

Table 4.8 Normalized emittance growth for different misalignments of the BTW accelerating sections $d_{c}$.

\begin{tabular}{|c|c|c|}
\hline & Short bunch & Medium bunch \\
\hline $\mathrm{d}_{\mathrm{c}}$ & $\varepsilon_{n, r m s}$ & $\varepsilon_{n, r m s}$ \\
\hline$[\mu \mathrm{m}]$ & {$[\mu \mathrm{rad}]$} & {$[\mu \mathrm{rad}]$} \\
\hline 50 & $0.10 \cdot 10^{-2}$ & 0.10 \\
100 & $0.38 \cdot 10^{-2}$ & 0.40 \\
150 & $0.86 \cdot 10^{-2}$ & 0.90 \\
200 & $1.53 \cdot 10^{-2}$ & 1.60 \\
250 & $2.39 \cdot 10^{-2}$ & 2.49 \\
300 & $3.44 \cdot 10^{-2}$ & 3.59 \\
500 & $9.55 \cdot 10^{-2}$ & 10.67 \\
\hline
\end{tabular}

\subsubsection{Control of the BBU induced banana shape}

The ensemble of trajectories including field errors, elements misalignment and trajectory correction plotted in Figure 4.3, Section 4.2.3 is here considered. The tracking is based on the pessimistic tolerance errors budget reported in Tables 4.2, 4.3 and 4.4, Section 4.2.3. The corresponding typical banana shapes induced by the BBU instability have been plotted at the Linac end in Figure 4.11. The banana shape is the representation of the transverse particle coordinate vs. the bunch duration; it is analytically described by the general solution (4.25). 
We associate at each banana a parameter defined by the transverse deviation of the bunch tail w.r.t. the head in units of (unperturbed) RMS beam size; it is called Ratio in Figure 4.11. It shows that the banana shape obtained after a simple trajectory correction in the Fermi Linac, hence without any particular approach to preserve the emittance, is about 6.5 times (RMS value) the RMS beam size over 600 fs bunch duration; its maximum excursion is $2 \mathrm{~mm}$ with respect the bunch head. This numbers would compromise the FEL process [50].
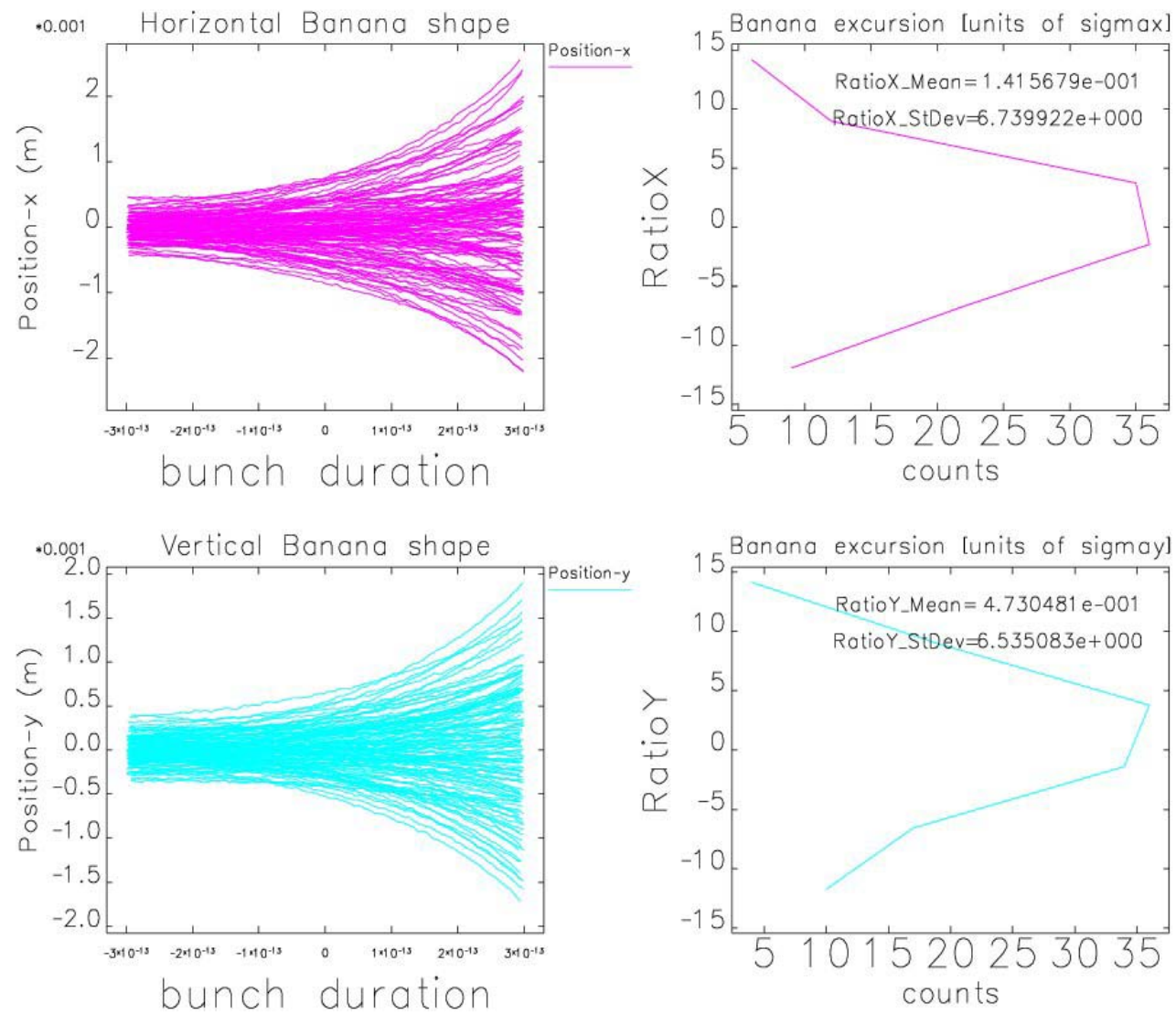

Figure 4.11 Banana shapes in the horizontal (top) and vertical (bottom) plane at the Linac end. Each curve in the left plots corresponds to one of the 120 trajectories shown in Figure 4.3, Section 4.2.3. On the right, the distribution of the amplitude of the banana shape, for each plane. The parameter Ratio_StDev is defined as the RMS transverse deviation of the bunch tail w.r.t. the head in unit of (unperturbed) RMS beam size.

For this reason a local bump has been applied at the beginning of the Linac region where the transverse wake fields are strongest. Figure 4.12 shows that the change of sign of the horizontal trajectory after the bump in the following structures allows for compensating the emittance dilution and for reducing the final banana shape to the level of $1-\sigma$. This means that a particular trajectory has been found so that all the kicks distributed along the Linac compensate each other. 

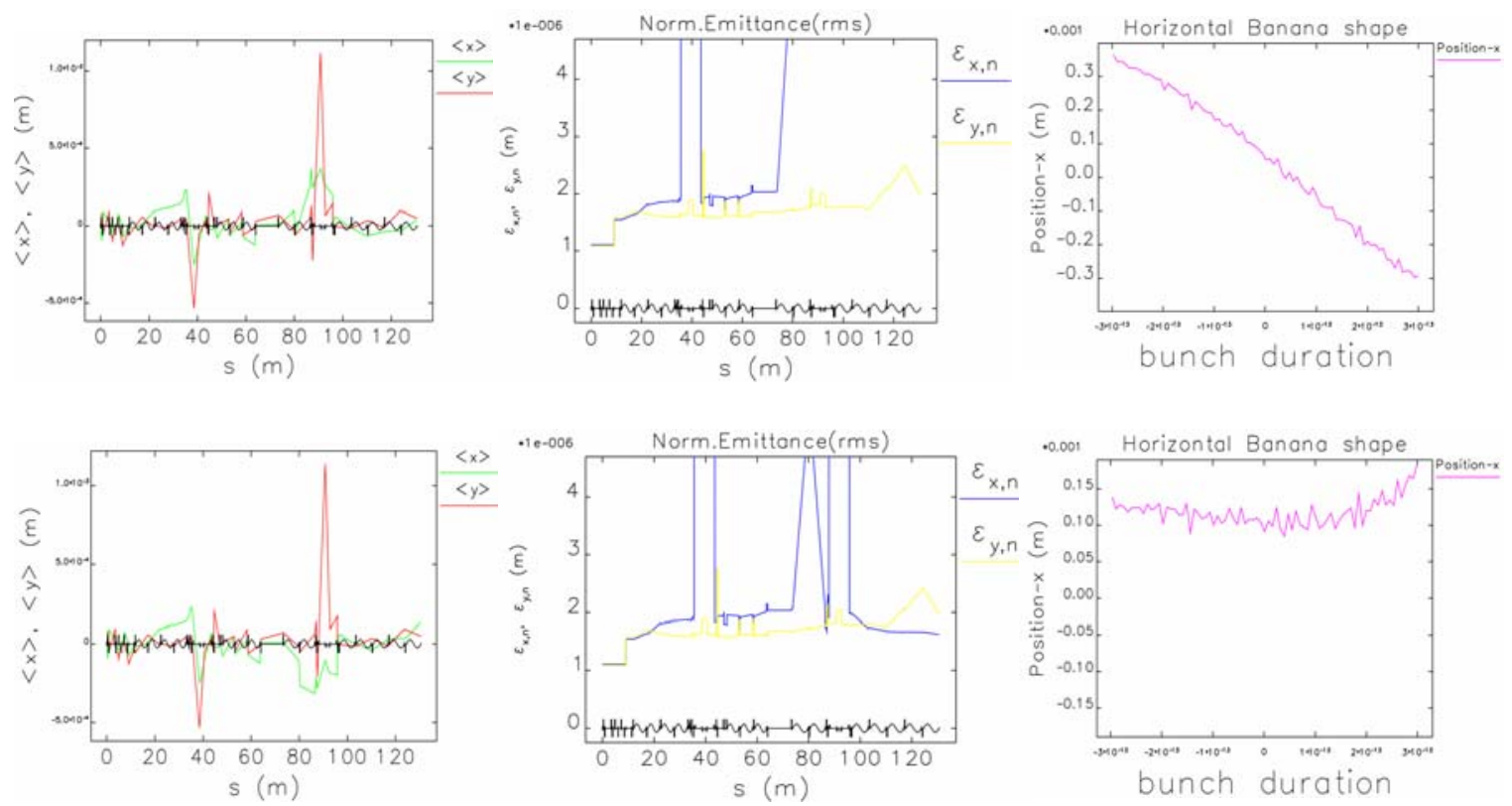

Figure 4.12 Evidence of BBU instability in the horizontal plane, before the local trajectory bump (top plots) and after the bump (bottom plots). From left to right: electron bunch trajectory, evolution of the normalized transverse emittance along the Linac and banana shape in the horizontal plane at the Linac end. Before the bump, the horizontal emittance exploits as the bunch enters the $\mathrm{S} 1$ accelerating structure (top centre); the final banana shape covers $600 \mu \mathrm{m}$ in the transverse plane (top right). After the bump, the horizontal trajectory (in green) changes its sign (bottom left) allowing for the emittance preservation until the Linac end (bottom centre); here, the banana shape is reduced to the level of $1-\sigma$ (bottom right).

\subsection{Effect of trajectory jitter on the banana shape}

Unfortunately, local methods of correction have the disadvantage to be dependent on the particular conditions of operation at their specific location; in this case, jitters may be a source of error of the local correction. For this reason, a study of the trajectory jitter generated by a jitter in the beam launching error - has been performed and jitter in the banana shape computed. Figure 4.13 demonstrates that a properly corrected banana shape is not dramatically affected from this jitter, since it remains below the $1-\sigma$ level. However, a non-local correction performed using many bpms and steerers distributed along the Linac results to be equally efficient and allows for reducing the maximum strength required by the steerers for the emittance preservation. 

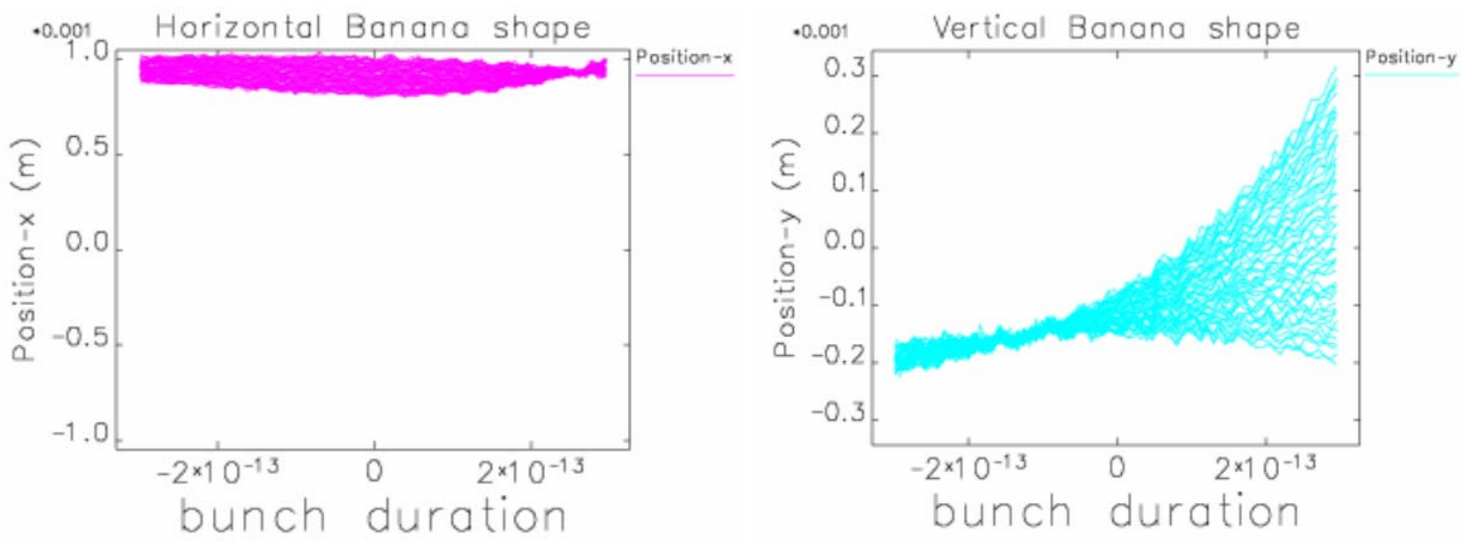

Figure 4.13 Effect of the launching error jitter on the banana shape at the end of the Linac. Initially, the BBU instability is completely suppressed in the horizontal plane (RatioX $<0.1$ ) and only partially in the vertical plane (RatioY $=2.3$ ) (not shown here). After adding the jitter, the trajectory bump is perturbed. The banana shape that was completely compensated still remains below the $1-\sigma$ level (RatioX_StDev $=0.4$, left plot), while the banana shape only partially compensated is now excited and degrades the transverse emittance (RatioY_StDev $=4.5$, right plot).

\subsection{Transversal acceptance and error tolerances}

\subsubsection{Beam size and aperture}

The optics in the Fermi Linac from the Injector end to the Spreader end is shown in Figure 4.14. Horizontal dispersion in the two chicanes corresponds to the bending angles for the compression of the Medium bunch. Table 4.9 shows that, regarding the beam size, the chromatic contribution to the emittance is the only important difference between the different machine setups and it is always much bigger than the geometrical one.

Table 4.9 Chromatic and geometrical contributions to the horizontal emittance in the dispersive regions of the Fermi Linac. A parallel Spreader has been considered here (see, Section 3.4)

\begin{tabular}{clc|c|c}
\hline Location & Beam Size & SHORT & MEDIUM & LONG \\
\hline \multirow{3}{*}{$\mathrm{BC} 1$} & $\eta_{\mathrm{x}} \sigma_{\delta}[\mathrm{mm}]$ & 3.1 & 5.7 & 4.0 \\
\cline { 2 - 5 } & $\sigma_{\beta}[\mathrm{mm}]$ & & 0.20 & \\
\hline \multirow{3}{*}{$\mathrm{BC} 2$} & $\eta_{\mathrm{x}} \sigma_{\delta}[\mathrm{mm}]$ & 0.7 & 1.9 & 2.6 \\
\cline { 2 - 5 } & $\sigma_{\beta}[\mathrm{mm}]$ & & 0.150 & \\
\hline \multirow{2}{*}{ SPREADER } & $\eta_{\mathrm{x}} \sigma_{\delta}[\mathrm{mm}]$ & & 0.130 \\
& $\sigma_{\beta}[\mathrm{mm}]$ & & 0.050 \\
\hline
\end{tabular}




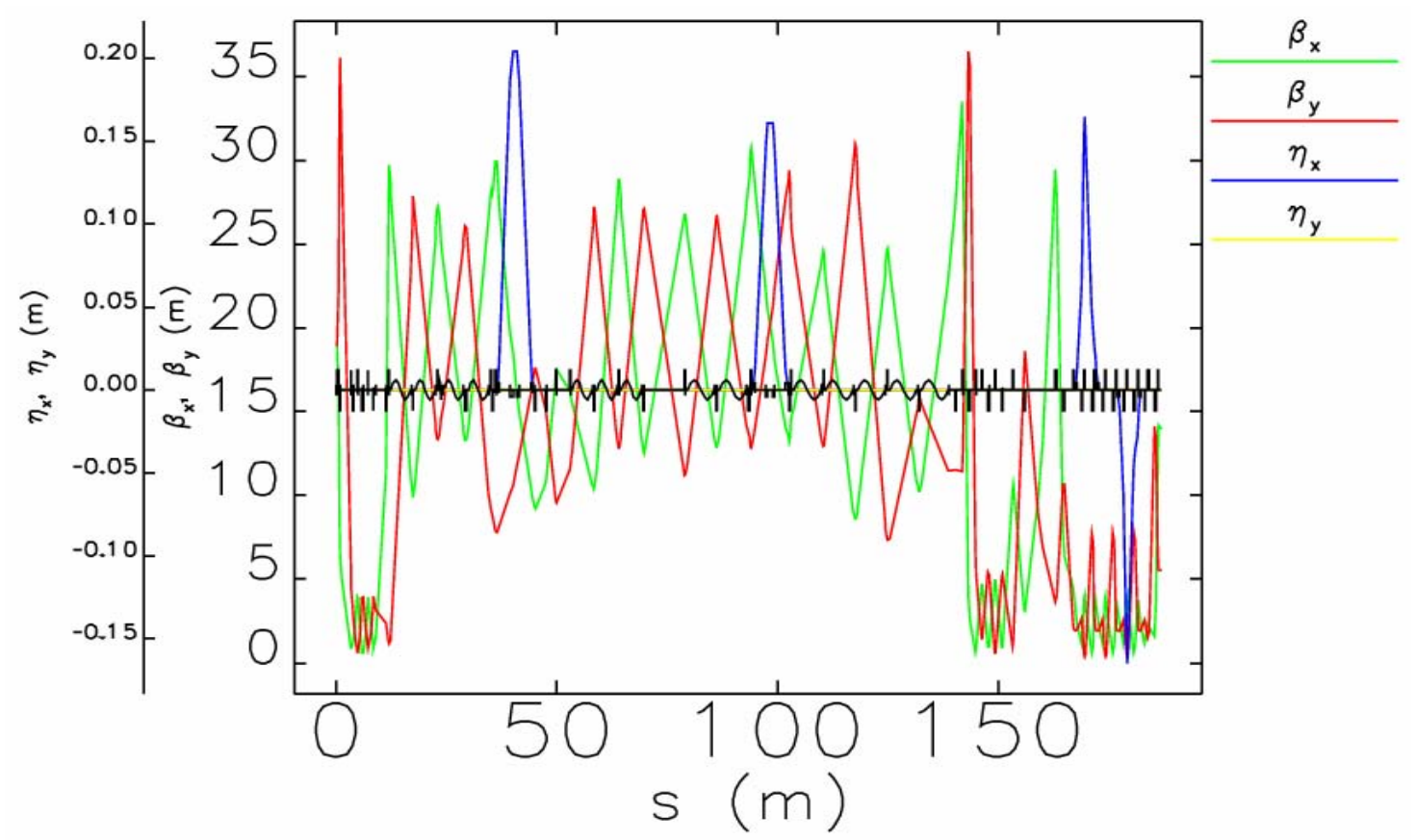

Figure 4.14 Fermi Linac optics from the Injector end (beam average energy is $95 \mathrm{MeV}$ ) to the Spreader end (beam average energy is $1180 \mathrm{MeV}$ ). Horizontal dispersion in the two magnetic chicanes is here determined by the compression parameters for the Medium bunch.

We now consider the geometrical $\left(\varepsilon_{g} \beta\right)$ and the chromatic $\left(\eta_{x} \sigma_{\delta}\right)$ contributions to the beam size and we define $u_{o a}$ the off-axis displacement of the bunch centroid (as it results after the trajectory correction); then, the "beam stay clear" condition will be defined by:

$$
h . b s c=1.2 \cdot\left(3 \sqrt{\varepsilon_{g} \beta+\left(\eta_{x} \sigma_{\delta}\right)^{2}}+u_{o a}\right)
$$

It includes a margin of error of $20 \%$ and defines to the half width of the vacuum chamber accommodating the beam along the Linac. An RMS geometrical emittance is here considered and a Gaussian transverse distribution of the particles coordinate is assumed. Figures 4.15 and 4.16 show respectively the behaviour along the Linac of the RMS geometrical emittances and of the RMS correlated energy spread (Medium bunch case). The incremental step of the emittances in the first part of the Linac is a trick implemented in the Elegant code to over-estimate their final value. Basing on results shown in Figure $4.14,4.15$ and 4.16, we can see that in dispersion free regions, h.bsc (4.27) assumes its maximum value in the horizontal plane at the beginning of the Linac:

$$
h . b s c=3.1 \mathrm{~mm} \text { (max. value in dispersion free regions) }
$$

We are considering an RMS normalized emittance $\varepsilon_{\mathrm{n}}=1.5 \mu \mathrm{m}$ and $u_{o a}=1 \mathrm{~mm}$. Because of the symmetry of optics in the two planes and of the round beam injected into the Linac, the same upper limit is still valid for the vertical plane. 


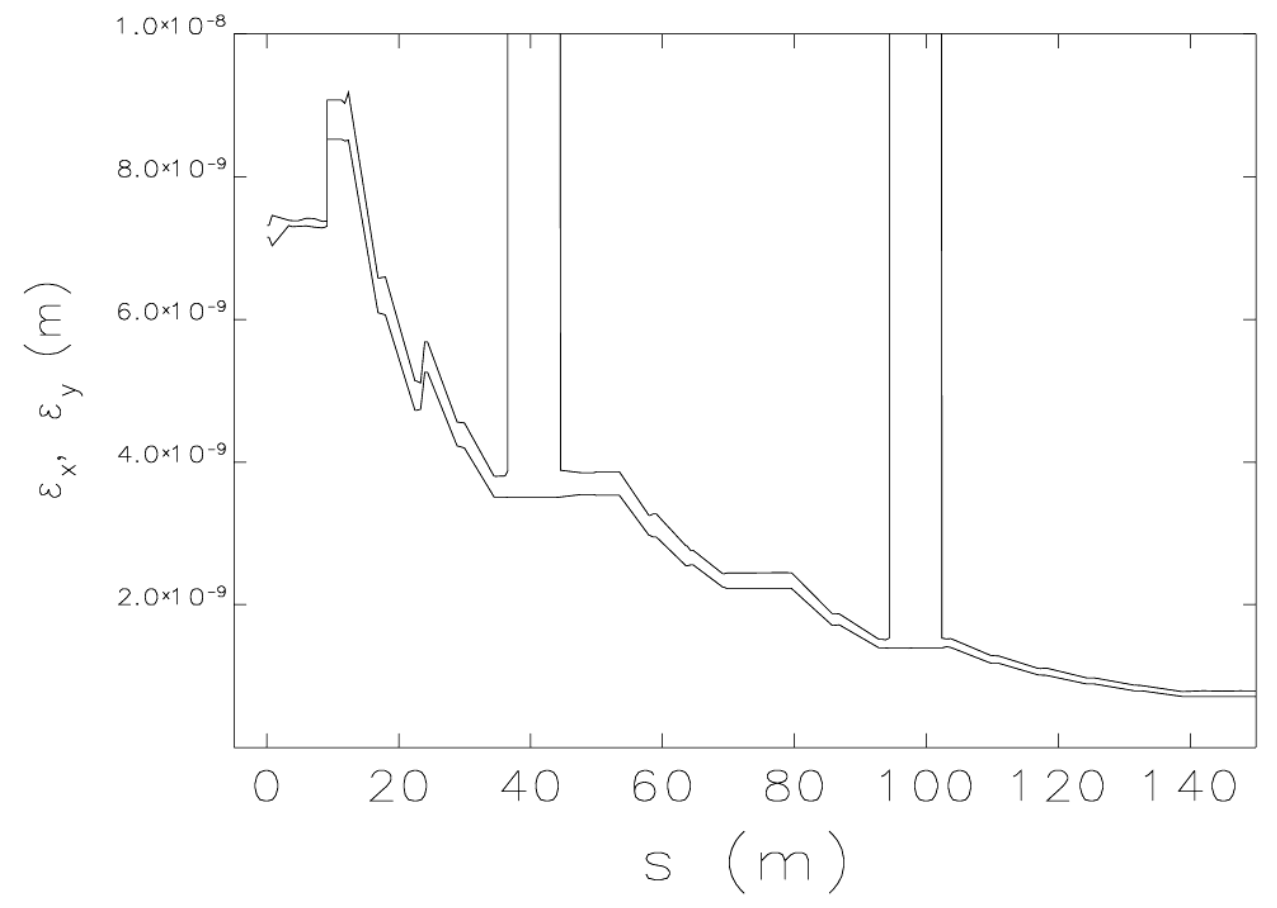

Figure 4.15 Behaviour of the RMS geometrical emittances along the Fermi Linac for the Medium bunch case. Chromatic contribution to the emittance is included, as shown by the fast growth in the dispersive regions of the chicanes.

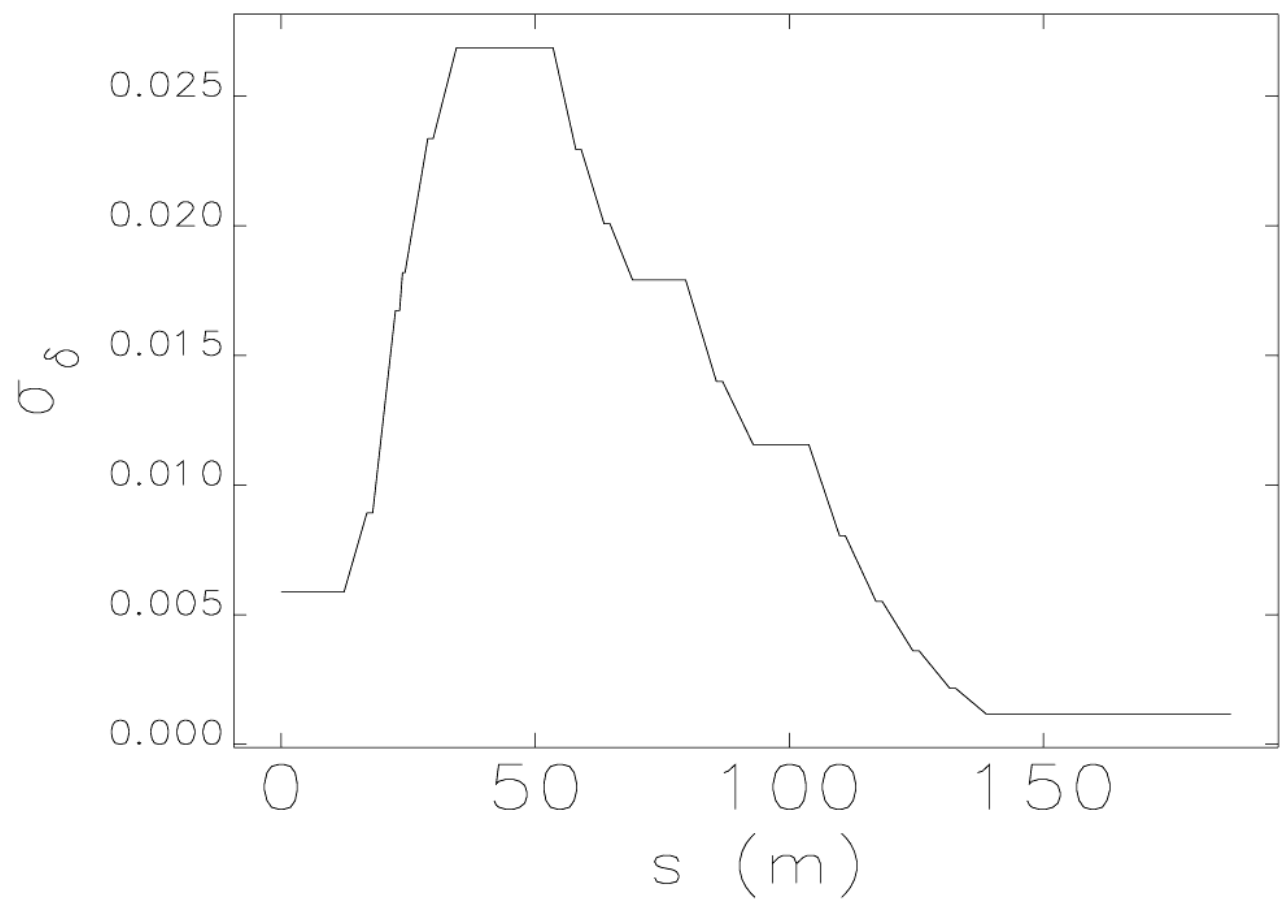

Figure 4.16 Behaviour of the correlated RMS energy spread along the Fermi Linac for the Medium bunch case. 
Special care has to be taken as for the horizontal motion in the chicanes. For the detailed evaluation of the width of the vacuum chamber in such regions we send to Section X. Briefly, we report here some results based on the consideration of two options: a fixed and large vacuum chamber and a smaller movable one. As for $\mathrm{BC} 1$, the maximum transverse displacement of the bunch centroid w.r.t the straight path is within the range $0.165 \mathrm{~m}<\eta_{\mathrm{x}}<0.255 \mathrm{~m}$. The total width of the vacuum chamber shall be:

$$
\begin{aligned}
& \text { fix_width }=13.0 \mathrm{~cm} \text { for the fixed option, } \\
& \text { mov_width }=4.5 \mathrm{~cm} \text { for the movable one. }
\end{aligned}
$$

As for $\mathrm{BC} 2$, the maximum transverse displacement of the bunch centroid w.r.t the straight path is within the range $0.156 \mathrm{~m}<\eta_{\mathrm{x}}<0.240 \mathrm{~m}$. The total width of the vacuum chamber shall be:

$$
\begin{aligned}
& \text { fix_width }=10.5 \mathrm{~cm} \text { for the fixed option, } \\
& \text { mov_width }=2.4 \mathrm{~cm} \text { for the movable one. }
\end{aligned}
$$

Referring to Table 4.7 and looking at Figure 4.14, we can calculate $h . b s c$ for the Spreader in presence and in absence of dispersion, respectively:

$$
h . b s c=1.7 \mathrm{~mm} \text { (max. value in dispersive regions) }
$$

\subsubsection{Misalignment}

$$
h . b s c=1.5 \mathrm{~mm} \text { (max. value in dispersion free regions) }
$$

Analytical calculations and simulations predict in agreement some tolerances on the alignment of the magnetic elements and of the accelerating structures in order to avoid emittance blow up (see, Section 4.2); they are summarized in Table 4.10. Such tolerances are not sufficient to avoid the BBU instability; nevertheless, it has been demonstrated (see, Section 4.3) that the instability can be counteracted by mean of a proper management of the trajectory correction. In general, suppression of BBU is made easier end more efficient as the misalignment of the elements in the lattice is reduced. Simulations suggest that the suppression of BBU without trajectory bumps should require beam based alignment technique in the Linac.

Tolerances on the launching error at the beginning of the Linac are given below; they include the contribution from the jitter in the bunch centroid position and divergence:

$$
\begin{gathered}
|\Delta \mathrm{x}|,|\Delta \mathrm{y}|<100 \mu \mathrm{m} \\
\left|\Delta \mathrm{x}^{\prime}\right|,\left|\Delta \mathrm{y}^{\prime}\right|<100 \mu \mathrm{rad}
\end{gathered}
$$

Table 4.10 Tolerances for elements misalignment, RMS values. They are consistent with BPMs resolution of $30 \mu \mathrm{m}$ RMS and with maximum correction strength of $2 \mathrm{mrad}$ provided by the steerers. 


\begin{tabular}{lccc}
\hline & $\Delta \mathbf{x}, \Delta \mathbf{y}$ & $\Delta \mathbf{z}$ & $\Delta \phi_{\text {Roll }}$ \\
\hline & {$[\mu \mathrm{m}]$} & {$[\mu \mathrm{m}]$} & {$[\mathrm{mrad}]$} \\
\hline DIPOLE & - & - & 0.8 \\
\hline QUADRUPOLE & 100 & 150 & 1.0 \\
\hline BPM & 100 & 150 & - \\
\hline ACC. STRUCT. & $300(100)$ & - & - \\
\hline
\end{tabular}

\subsubsection{Field quality}

Results from Section 4.2 are here summarized in Table 4.11-14 in form of tolerances for the magnetic field quality, in order to reduce the emittance blow up due to filamentation below the 1\% level. Multipolar components [51] for the dipole magnets are evaluated at a radius $\mathrm{R}=20 \mathrm{~mm} ; \mathrm{b}_{\mathrm{n}, 0}$ is the main field component according to the index of the magnet $(\mathrm{n}=0$ for dipole, $\mathrm{n}=1$ for quadrupole, etc...).

Table 4.11 Tolerances for the main field component of dipoles and quadrupoles magnet.

\begin{tabular}{ll}
\hline & $\left|\Delta \mathbf{b}_{\mathbf{n}} / \mathbf{b}_{\mathbf{n}, 0}\right|$ \\
\hline DIPOLE BC1 & $1 \times 10^{-5}$ \\
\hline DIPOLE BC2 & $1 \times 10^{-5}$ \\
\hline DIPOLE SPREADER & $1 \times 10^{-5}$ \\
\hline QUADRUPOLE & $1 \times 10^{-4}$ \\
\hline
\end{tabular}

Table 4.12 Field quality tolerances in $\mathrm{BC} 1$, RMS values. $\theta_{\mathrm{b}}=0.085 \mathrm{rad}, \sigma_{\delta}=2.8 \%, \gamma=450$.

\begin{tabular}{lcccc}
\hline & B11 & B12 & B13 & B14 \\
\hline$\beta_{\mathrm{x}}[\mathrm{m}]$ & 30 & 20 & 15 & 10 \\
\hline$\eta_{\mathrm{x}}[\mathrm{m}]$ & $\leq 0.02$ & 0.25 & 0.25 & $\leq 0.02$ \\
\hline$\left|\mathrm{b}_{1} / \mathrm{b}_{\mathrm{n}, 0}\right|[\%]$ & 0.06 & 0.006 & 0.007 & 0.11 \\
\hline$\left|\mathrm{b}_{2} / \mathrm{b}_{\mathrm{n}, 0}\right|[\%]$ & 1.5 & 0.012 & 0.014 & 2.8 \\
\hline$\left|\mathrm{b}_{4} / \mathrm{b}_{\mathrm{n}, 0}\right|[\%]$ & 100 & 0.05 & 0.06 & 100 \\
\hline
\end{tabular}

Table 4.13 Field quality tolerances in BC2, RMS values. $\theta_{\mathrm{b}}=0.080 \mathrm{rad}, \sigma_{\delta}=1.0 \%, \gamma=1174$.

$\begin{array}{llll}\text { B21 } & \text { B22 } & \text { B23 } & \text { B24 }\end{array}$




\begin{tabular}{lcccc}
\hline$\beta_{\mathrm{x}}[\mathrm{m}]$ & 17 & 14 & 11 & 10 \\
\hline$\eta_{\mathrm{x}}[\mathrm{m}]$ & $\leq 0.02$ & 0.22 & 0.22 & $\leq 0.02$ \\
\hline$\left|\mathrm{b}_{1} / \mathrm{b}_{\mathrm{n}, 0}\right|[\%]$ & 0.15 & 0.015 & 0.018 & 0.20 \\
\hline$\left|\mathrm{b}_{2} / \mathrm{b}_{\mathrm{n}, 0}\right|[\%]$ & 10.6 & 0.10 & 0.11 & 14.1 \\
\hline$\left|\mathrm{b}_{4} / \mathrm{b}_{\mathrm{n}, 0}\right|[\%]$ & 100 & 0.5 & 0.5 & 100 \\
\hline
\end{tabular}

Table 4.14 Field quality tolerances in the Spreader (option of four dipoles for parallel undulators lines), RMS values. $\theta_{\mathrm{b}}=0.055 \mathrm{rad}, \sigma_{\delta}=0.1 \%, \gamma=2348$.

\begin{tabular}{lcc}
\hline & BS1, BS3 & BS2, BS4 \\
\hline$\beta_{\mathrm{x}}[\mathrm{m}]$ & 3 & 2 \\
\hline$\eta_{\mathrm{x}}[\mathrm{m}]$ & 0.1 & $\leq 0.01$ \\
\hline$\left|\mathrm{b}_{1} / \mathrm{b}_{\mathrm{n}, 0}\right|[\%]$ & 0.8 & 9.1 \\
\hline$\left|\mathrm{b}_{2} / \mathrm{b}_{\mathrm{n}, 0}\right|[\%]$ & 100 & 100 \\
\hline$\left|\mathrm{b}_{4} / \mathrm{b}_{\mathrm{n}, 0}\right|[\%]$ & 100 & 100 \\
\hline
\end{tabular}

Tolerances for the multipolar field components for $\mathrm{BC} 1$ are a factor $(2.5)^{\mathrm{n}}$ stronger than those for LCLS because of the larger energy spread foreseen for Fermi $\left(\sigma_{\delta}=2.8 \%\right)$; tolerances for dipoles in $\mathrm{BC} 2$ are comparable to those for LCLS, instead. Field quality for dipoles in the Spreader is much more relaxed than in the BCs. In all cases the tolerances result to be pretty relaxed, they have been set to $100 \%$.

\subsubsection{Optical mismatch at the injection}

Analytical calculations in Section 4.2 and preliminary results from jitter studies in timedependent simulations suggest some tolerances on the optical mismatch of the electron beam phase space with the Twiss ellipse at the beginning of the Linac. They are listed below, in order to avoid an emittance dilution larger than 10\% (pessimistic scenario):

$$
\left(\frac{\Delta \beta}{\beta_{0}}\right)_{i}<3 \% \quad \text { and } \quad\left\{\begin{array}{c}
\widetilde{\eta}_{i}<1 \mathrm{~mm} \\
\widetilde{\eta}_{i}^{\prime}<0.5 \mathrm{mrad}
\end{array}\right.
$$

\section{Tracking studies without errors}

For particle tracking through the accelerator we used three codes: LiTrack [16], Elegant [23] and IMPACT [24] (see also Attachment B). Typically, the initial plan for a 
formation of the electron bunch at the end of the accelerator was made analytically and then was tested and re-optimized with LiTrack. As was mentioned before, the transverse particle motion at this stage of the accelerator design was not important. Then, the LiTrack simulations were followed with simulations performed using Elegant and recently with IMPACT using completed machine lattice. The output longitudinal distribution produced by all three codes (as well as the evolution of this distribution along the accelerator) always agreed rather accurately (see, Figure (5.1)). This confirms our original estimation that CSR and LSC wake potentials contribute only weakly to the formation of the electron bunch on a large scale comparable to the bunch length. We note that LiTrack simulation does not include CSR and LSC. Occasionally, minor tweaking of the RF settings in Elegant was required to compensate for small differences caused by the CSR wakes which, actually, were overestimated in simulations because the effect of the shielding existing on a large scale was not included there.
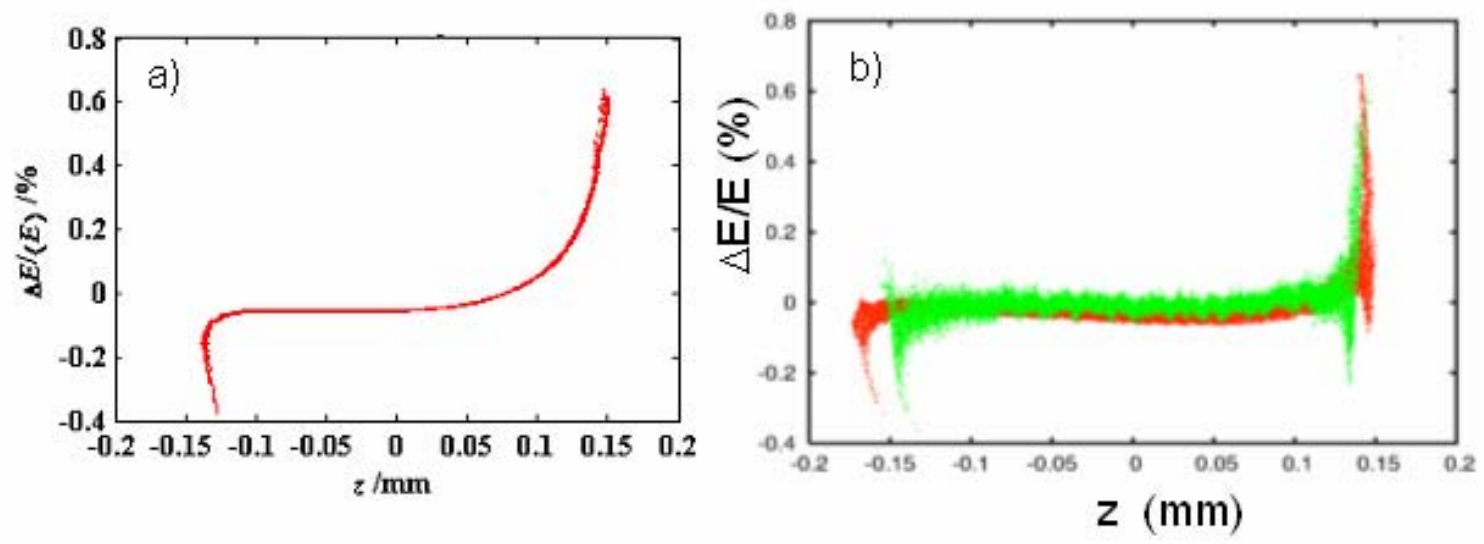

Figure (5.1) The plot of the longitudinal phase space for the medium bunch case with ramped peak current in the injector: a) tracking with LiTrack using initial rms energy spread of $2 \mathrm{keV}, \mathrm{b}$ ) tracking with Elegant (green) and IMPACT (red) using initial rms energy spread of $10 \mathrm{keV}$

In all tracking studies with Elegant and IMPACT we used the output of the injector simulations. However, we artificially scaled the average emittance to approximately 1.5 $\mathrm{mm}$-mrad in anticipation that a true emittance will likely be worse than produced in the injector simulations. In most cases the initial distribution had variations of the slice emittance along the electron bunch and we preserved these variations in the emittance scalling. Finally, we did not model the laser heater and simply assumed that it creates the uncorrelated Gaussian distribution of electron energies. In this case we assumed that this distribution dominates the original distribution from the injector and use the same slice energy distribution over the entire bunch (excluding edges).

Performing simulation with Elegant we switched off LSC effect because of the problem with the numerical noise described before. We considered including LSC into simulations with IMPACT, but at the time of writing this part of the IMPACT is still in the testing mode. Typically we repopulated particle distribution produced in the injector up to $2 \mathrm{M}$ macro particles for simulations with Elegant and up to $20 \mathrm{M}$ macro particles for simulations with IMPACT. This re-populating preserved all global characteristics of the original distribution. 


\subsection{Short bunch case}

Short bunch case was primarily designed for a beginning of the machine commissioning with a relatively small bunch charge. The list of the electron beam parameters for this case is given in Table (5.1). All settings for RF voltages and phases for all four linac phases and for values of $R_{56}$ in the two bunch compressors are given in the Table (5.4) which is a joint table for three bunch configurations.

Table (5.1). Electron beam parameters for a short bunch case.

\begin{tabular}{|l|c|l|}
\hline Bunch charge & 0.33 & $\mathrm{nC}$ \\
Beam energy & 1.2 & $\mathrm{GeV}$ \\
Peak current (beam core) & 800 & $\mathrm{~A}$ \\
Bunch duration (full width, beam core) & 200 & $\mathrm{fs}$ \\
Slice energy spread (rms, beam core) & 150 & $\mathrm{keV}$ \\
Slice emittance (rms, beam core) & 1.5 & $\mu \mathrm{m}$ \\
Laser heater (energy spread rms) & 8 & $\mathrm{keV}$ \\
Compression factor in BC1 & 3.0 & \\
Compression factor in BC2 & 3.5 & \\
\hline
\end{tabular}

The electron distribution in the longitudinal phase space and a histogram of the peak current at the end of the accelerator are shown in Figure (5.1) and (5.2).

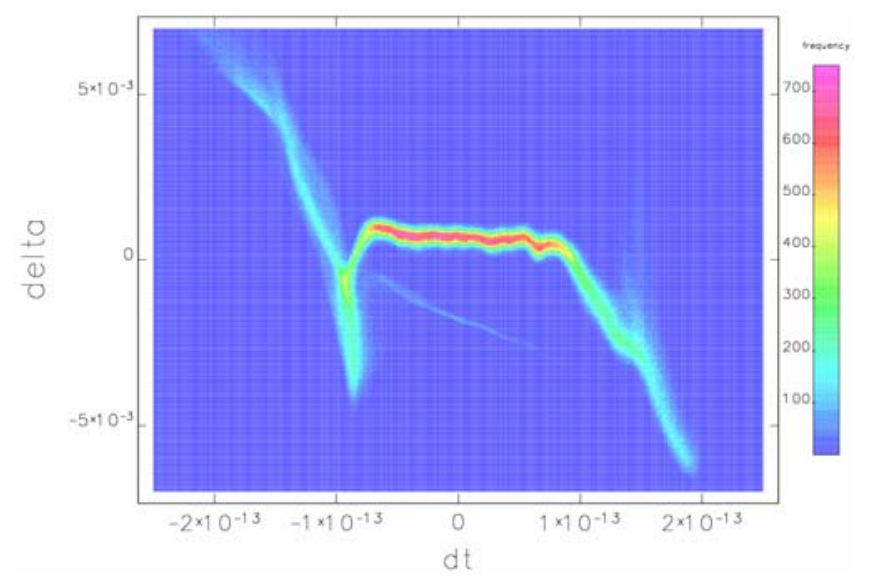

Figure (5.1) Density plot showing the distribution of the electron relative energy spread versus time for a short bunch case. 


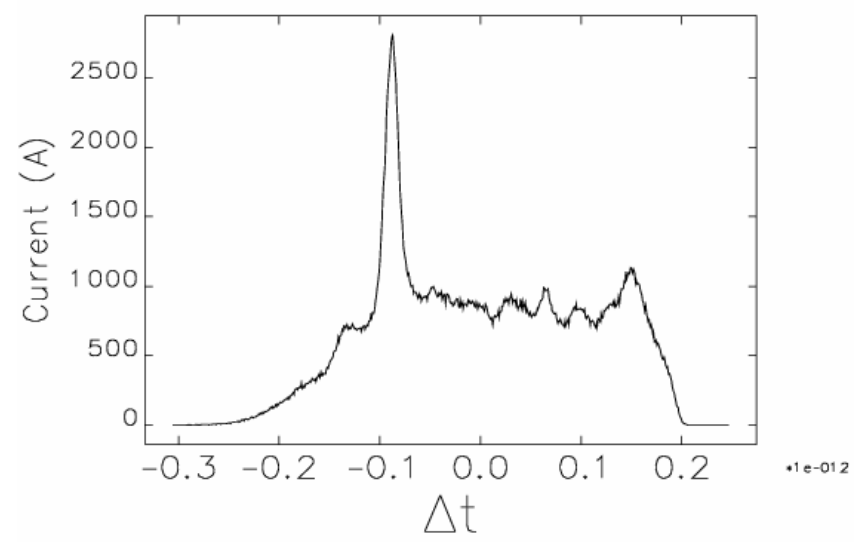

Figure 8: A histogram of the electron beam peak current.

\subsection{Medium bunch case}

The electron beam parameters for a medium bunch case are given in the Table (5.2). Except for the bunch charge and the bunch length they are very similar to the parameters in the short bunch case. We also add to the table a new parameter flatness which we use to characterize the quadratic energy chirp in the electron distribution at the end of the accelerator. Two numbers in the table correspond to the two variants of the medium bunch case discussed below.

Table (5.2). Electron beam parameters for a medium bunch case.

\begin{tabular}{|l|c|l|}
\hline Bunch charge & 0.8 & $\mathrm{nC}$ \\
Beam energy & 1.2 & $\mathrm{GeV}$ \\
Peak current (beam core) & 800 & $\mathrm{~A}$ \\
Bunch duration (full width, beam core) & 700 & $\mathrm{fs}$ \\
Slice energy spread (rms, beam core) & 150 & $\mathrm{keV}$ \\
Slice emittance (rms, beam core) & 1.5 & $\mu \mathrm{m}$ \\
Laser heater (energy spread rms) & 9 & $\mathrm{keV}$ \\
Compression factor in BC1 & $\sim 4.0$ & \\
Compression factor in BC2 & 2.5 & \\
Flatness & $10 / 0.2$ & $\mathrm{MeV} / \mathrm{ps}^{2}$ \\
& & \\
\hline
\end{tabular}

Figures (5.3) and (5.4) show the electron distribution in the longitudinal phase space and a histogram of the peak current for two variants M2_GPT_08_8_10_10nov05 and M6_GPT_08_17_14may19 obtained with Elegant. All detail regarding these configurations are given in the Table (5.4). The first variant was prepared using the standard parabolic peak current distribution in the injector and the second variant was prepared using the ramped peak current distribution in the injector. Figure (5.5) show the plot of the slice emittance at the beginning of the accelerator and at the end of the accelerator for M6_GPT_08_17_14may19. According to this plot, there is no growth of slice emittance during acceleration and compression. It was observed not only in this 
case, but in all other case including long bunch cases. The variation of the slice emittance seen in Figure (5.5) is due to the ramped peak current in the injector. We note that the smallest emittance is at the head of the electron bunch. This can have a useful implication because head electrons radiate in the final stage of the harmonic cascade FEL with toughest requirement to the electron beam emittance.
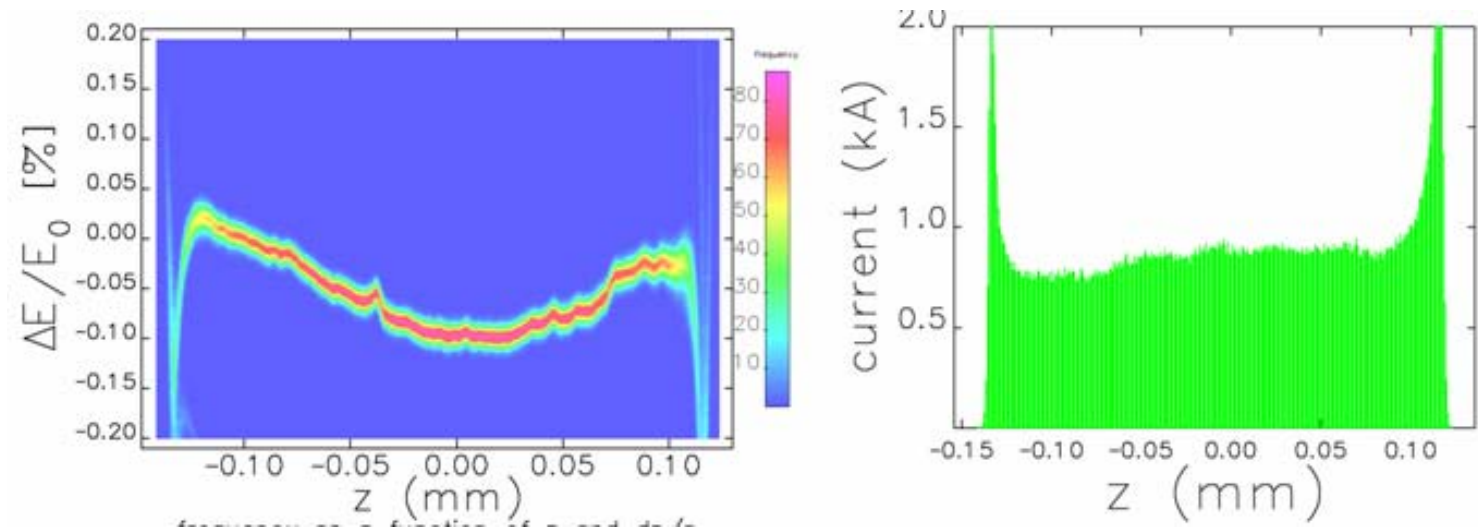

Figure (5.3) Variant M2_GPT_08_8_10_10nov05. Density plot showing the distribution of the electron relative energy spread versus time and histogram of the peak current.
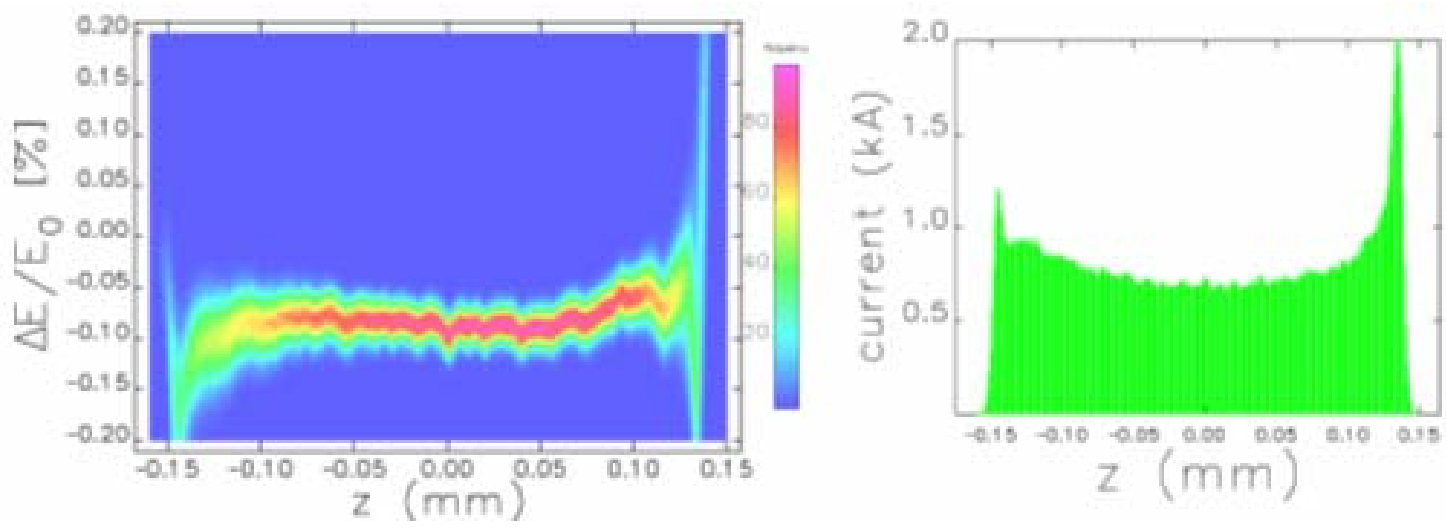

Figure (5.4) Variant M6_GPT_08_17_14may19. Density plot showing the distribution of the electron relative energy spread versus time and the histogram of the peak current.
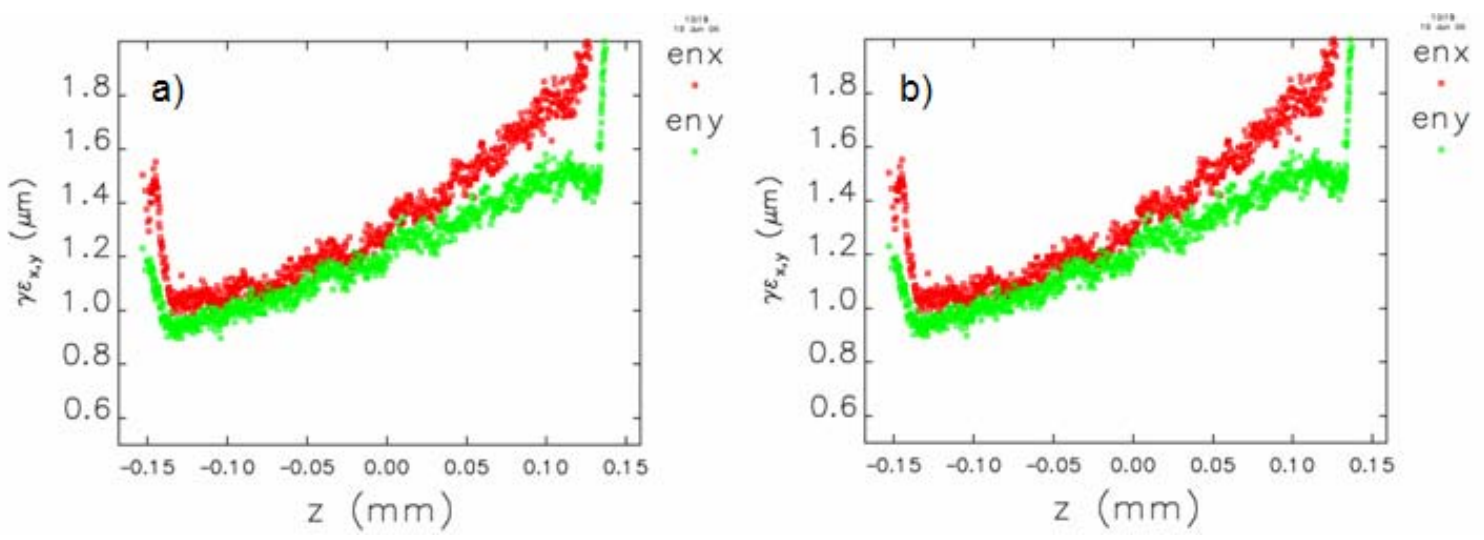
Figure (5.5) Slice emittance at the beginning (a) and at the end of the accelerator (b) for the variant M6_GPT_08_17_14may19.

\subsection{Long bunch case}

The electron beam parameters for a long bunch case are given in the Table (5.3) where two numbers for flatness correspond to the two variants discussed below.

Table (5.3). Electron beam parameters for a long bunch case.

\begin{tabular}{|l|c|l|}
\hline Bunch charge & 1.0 & $\mathrm{nC}$ \\
Beam energy & 1.2 & $\mathrm{GeV}$ \\
Peak current (beam core) & 500 & $\mathrm{~A}$ \\
Bunch duration (full width, beam core) & 1400 & $\mathrm{fs}$ \\
Slice energy spread (rms, beam core) & 150 & $\mathrm{keV}$ \\
Slice emittance (rms, beam core) & 1.5 & $\mu \mathrm{m}$ \\
Laser heater (energy spread rms) & 12 & $\mathrm{keV}$ \\
Compression factor in BC1 & $\sim 2.0$ & \\
Compression factor in BC2 & $\sim 3.0$ & \\
Flatness & $3 / 0.2$ & $\mathrm{MeV} / \mathrm{ps}^{2}$ \\
& & \\
\hline
\end{tabular}

Figures (5.6) and (5.7) show the electron distribution in the longitudinal phase space and a histogram of the peak current for two variants L2_Astra2_1_12_16_24jan06 and L5_... obtained with Elegant. All detail regarding these configurations are given in the Table (5.4). The first variant was prepared using the standard parabolic peak current distribution in the injector and the second variant was prepared using the ramped peak current distribution in the injector. Figure (5.8) show the plot of the slice emittance at the end of the accelerator for L6_Astra2_1_12_16_9june 06 .
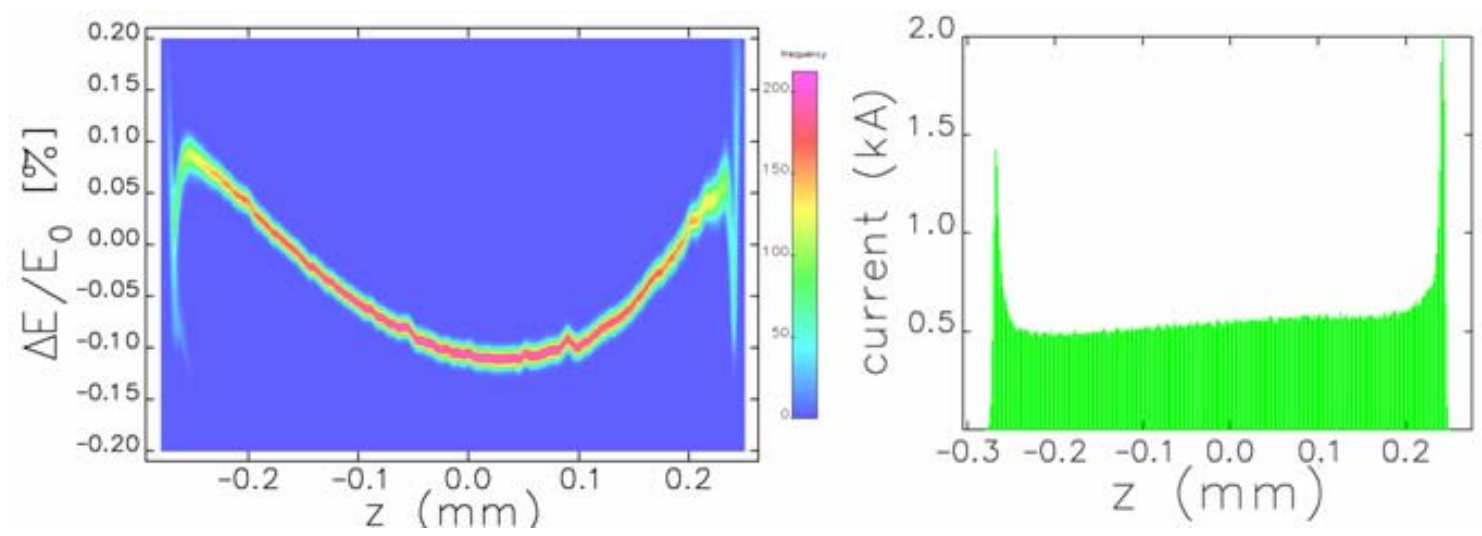

Figure (5.6) Variant L2_Astra2_1_12_16_24jan06. Density plot showing the distribution of the electron relative energy spread versus time and histogram of the peak current. 

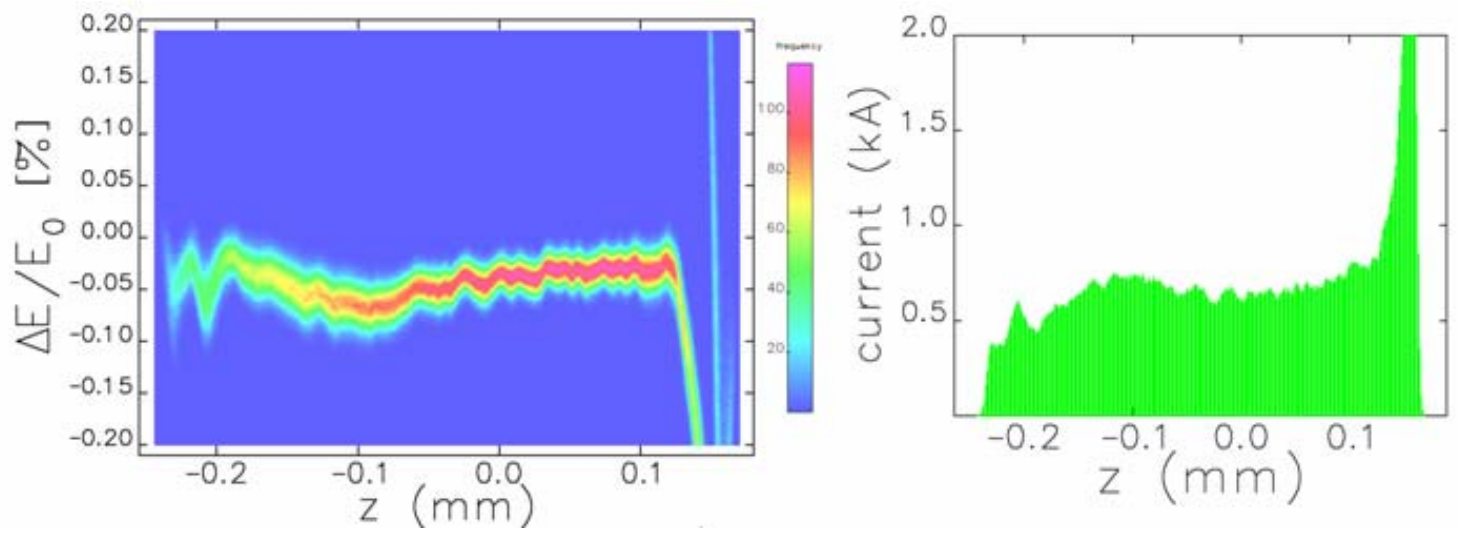

Figure (5.7) Variant L6_Astra2_1_12_16_9june06. Density plot showing the distribution of the electron relative energy spread versus time and the histogram of the peak current.

a)

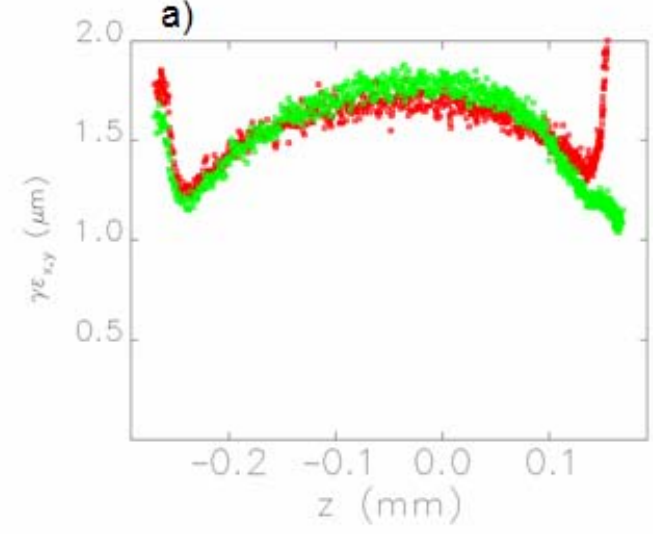

b)

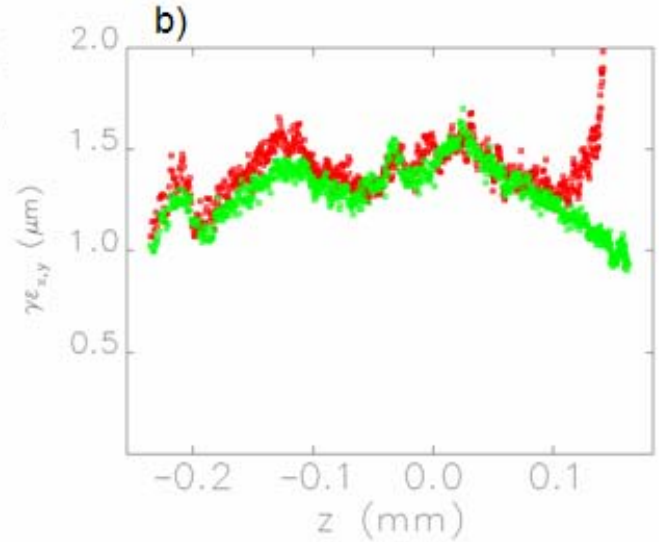

Figure (5.8) Slice emittance for a variant L2_Astra2_1_12_16_24jan06 (a) and for a variant L6_Astra2_1_12_16_9june06 (b).

Table (5.4). Basic linac and compressor parameters.

\begin{tabular}{|c|c|c|c|c|c|}
\hline Configuration & $\begin{array}{c}\text { Short } \\
\text { standard }\end{array}$ & $\begin{array}{l}\text { Medium } \\
\text { standard }\end{array}$ & $\begin{array}{l}\text { Medium } \\
\text { ramped }\end{array}$ & $\begin{array}{c}\text { Long } \\
\text { standard }\end{array}$ & $\begin{array}{l}\text { Long } \\
\text { ramped }\end{array}$ \\
\hline Output file name & $\begin{array}{c}\text { S3_GPT } \\
4 \_043 \_8 \\
-15 \_70 \mathrm{oc} \\
\text { t05 }\end{array}$ & $\begin{array}{c}\text { M2_GPT } \\
08 \_8 \_10_{-} 1 \\
\text { 0nov05 }\end{array}$ & $\begin{array}{c}\text { M6_GPT } \\
08 \_17 \_14 \\
\text { may19 }\end{array}$ & $\begin{array}{l}\text { L2_Astra2 } \\
1 \_12 \_16 \_24 \\
\text { jan06 }\end{array}$ & $\begin{array}{l}\text { L6_Astra2 } \\
\text {-1_12_16 } \\
\text { _-jjune06 }\end{array}$ \\
\hline Injector file name & $\begin{array}{l}\text { GPT200 } \\
\text { kp_4_5p } \\
\text { s330p } \\
4\end{array}$ & $\begin{array}{c}\text { GPT200kp_- } \\
\text { 9ps800pC_- } \\
\text { S0B_2 } \\
\end{array}$ & $\begin{array}{c}\text { Medium_ra } \\
\text { mp_n7wak } \\
\text { es }\end{array}$ & $\begin{array}{c}\text { Astra200kp_ } \\
\text { 10ps1nC_2_ } \\
\text { wakes }\end{array}$ & $\begin{array}{c}\text { Astra200kp } \\
-10 p s 1000 \\
\text { pC_5 }\end{array}$ \\
\hline Charge, $\mathrm{nC}$ & 0.33 & 0.8 & 0.8 & 1 & 1 \\
\hline Linac-1: voltage (MV) & $47 \times 4$ & $47 \times 4$ & $47 \times 4$ & $47 \times 4$ & $47 \times 4$ \\
\hline Linac-1: phase (deg) & 55.6 & 54 & 54 & 46.25 & 65 \\
\hline
\end{tabular}




\begin{tabular}{|l|r|r|r|r|r|} 
X-band: voltage (MV) & 19.1 & 17 & 18 & 19.1 & -18 \\
\hline X-band: phase (deg) & -90 & -88 & -90 & 225 & 90 \\
\hline Linac-2: voltage (MV) & $47 \times 3$ & $47 \times 3$ & $47 \times 3$ & $47 \times 3$ & $47 \times 3$ \\
\hline Linac-2: phase (deg) & 90 & 70 & 70 & 85 & 72 \\
\hline Linac-3: voltage (MV) & $120 \times 2$ & $120 \times 2$ & $120 \times 2$ & $120 \times 2$ & $120 \times 2$ \\
\hline Linac-3: phase (deg) & 90 & 70 & 70 & 75 & 72 \\
\hline Linac-4: voltage (MV) & $120 \times 5$ & $120 \times 5$ & $120 \times 5$ & $120 \times 5$ & $120 \times 5$ \\
\hline Linac-4: phase (deg) & 90 & 70 & 109 & 72 & 109 \\
\hline BC1: R56 (m) & -0.0285 & -0.026 & -0.028 & -0.026 & -0.028 \\
\hline BC2: R56 (m) & 0 & -0.0162 & -0.016 & -0.0395 & -0.034 \\
\hline
\end{tabular}

\section{Effect of errors}

This paragraph addresses jitter studies in the various configurations of the FERMI@ELETTRA linac, such as medium and long bunch cases. In fact, FEL operations foresee stringent requirements for the stability of the linac output parameters: electron bunch arrival time, relative peak current and relative mean energy. In order to understand the sensitivity of these parameters to jitters of various error sources along the linac an elaborate study using tracking codes has been performed. In paragraph 6.1 we describe effect of errors on longitudinal dynamics and as a result we created a tolerance budget to be used as guidance in the design of the linac upgrade. Furthermore we report slice jitter analyses on flatness of the longitudinal phase space that is another critical parameter in FEL process.

In paragraph 6.2 we present tracking studies with errors using Elegant code to generate input files for FEL simulations...

The tolerance budget has been also adopted as a collection of rms values for input parameters to perform global jitter analysis by means of Elegant code [24]. In this way the results between LiTrack and Elegant are compared.

\subsection{Effect of errors on longitudinal dynamics}

For sensitivities studies we have used LiTrack code [16]. It is a macro-particle fast tracking program that follows longitudinal position and relative energy deviation of the particles. LiTrack handles linac acceleration and bunch compression. Acceleration is applied as a sinusoidal variation and bend systems are described simply by their $1^{\text {st }}, 2^{\text {nd }}$, and $3^{\text {rd }}$ order path length vs. energy coefficients (R56, T566, and U5666) and the nominal energy. Wakefields of accelerating structures are included by convolving a point charge wake with the evolving temporal distribution. Figure (6.1) shows the FERMI@ELETTRA layout which is mainly composed by four Linacs, from Linac 1 to Linac 4, and two bunch compressors (BC1 and $\mathrm{BC} 2$ ). Layout also foresees a X-band cavity as linearizer for longitudinal phase space and a laser heater to suppress microbunching instability (see section 2). 
FEL operations presume different options for the electron bunch length which foresee different configurations for parameters in the FERMI@ELETTRA. Here we present studies for medium (M) and long (L) bunch length. In addition, each option foresees a different particle distribution out of photo-injector: with parabolic (M2 and L2) and ramped (M6 and L4) peak currents. In Table (6.1) we collect nominal compression, $\mathrm{R}_{56}$ terms in bunch compressions (BC), and acceleration parameters, amplitude and phase of accelerating sections in linacs, used in longitudinal tracking simulations and sensitivity studies.

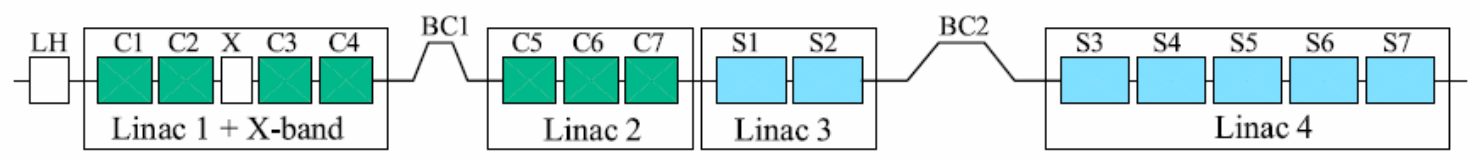

Figure (6.1) Layout of the FERMI@ELETTRA linac used in sensitivity studies with LiTrack.

Table (6.1) Nominal parameters, compression ( $\mathrm{BC} 1$ and $\mathrm{BC} 2$ ) and acceleration, for different configurations of the FERMI@ELETTRA linac: medium bunch cases M2 and M6 and long bunch cases L2 and L4. Bunch charge is $800 \mathrm{pC}$ and $1 \mathrm{nC}$ respectively for long and medium bunch case. In parenthesis values of $\mathrm{R}_{56}$ used in Elegant. Phase reference is 0 deg for "on crest".

\begin{tabular}{|c|c|c|c|c|c|c|}
\hline Parameters & Sy. & M2 & M6 & L2 & $\mathrm{L4}$ & Unit \\
\hline Laser heater & $\overline{\sigma_{\mathrm{dE} / \mathrm{E}}}$ & $8 \cdot 10^{-5}$ & $1.5 \cdot 10^{-4}$ & $1.2 \cdot 10^{-4}$ & $1.2 \cdot 10^{-4}$ & \\
\hline L1 RF voltage & $\mathrm{V}_{1}$ & 188 & 188 & 188 & 188 & $\mathrm{MeV}$ \\
\hline L1 RF phase & $\phi_{1}$ & -36 & -36 & -39 & -25 & S-band deg \\
\hline LX RF voltage & $\mathrm{V}_{\mathrm{x}}$ & 17 & 18 & 14 & 18 & $\mathrm{MeV}$ \\
\hline LX RF phase & $\phi_{\mathrm{x}}$ & 182 & 180 & 135 & 180 & X-band deg \\
\hline L2 RF voltage & $\mathrm{V}_{2}$ & 141 & 141 & 141 & 141 & $\mathrm{MeV}$ \\
\hline L2 RF phase & $\phi_{2}$ & -20 & -20 & -5 & -18 & S-band deg \\
\hline L3 RF voltage & $\mathrm{V}_{3}$ & 240 & 240 & 240 & 240 & $\mathrm{MeV}$ \\
\hline L3 RF phase & $\phi_{3}$ & -20 & -20 & -15 & -18 & S-band deg \\
\hline L4 RF voltage & $\mathrm{V}_{4}$ & 600 & 600 & 600 & 600 & $\mathrm{MeV}$ \\
\hline L4 RF phase & $\phi_{4}$ & -20 & 19 & 0 & 5 & S-band deg \\
\hline BC1 & $\mathrm{R}_{56 \_1}$ & $\begin{array}{c}-2.67 \\
(-2.60)\end{array}$ & $\begin{array}{c}-2.87 \\
(-2.80)\end{array}$ & $\begin{array}{c}-2.77 \\
(-2.60)\end{array}$ & $\begin{array}{c}-2.95 \\
(-2.80)\end{array}$ & $\begin{array}{l}\mathrm{cm} \\
\mathrm{cm}\end{array}$ \\
\hline BC2 & $\mathrm{R}_{56 \_2}$ & $\begin{array}{c}-1.65 \\
(-1.62)\end{array}$ & $\begin{array}{c}-1.67 \\
(-1.60)\end{array}$ & -3.70 & -3.40 & $\begin{array}{l}\mathrm{cm} \\
\mathrm{cm}\end{array}$ \\
\hline
\end{tabular}

\subsubsection{Simulation technique}

In this part we describe the simulation technique used for sensitivity studies. In the case of small jitter errors we can consider the dependence between input random variable $\mathrm{X}$ and output parameters $\mathrm{Y}$ as: 


$$
Y=f(X)=c X^{2}+b X+a
$$

where $\mathrm{X}$ is random variable normally distributed with density of probability $\mathrm{p}_{\mathrm{x}}(\mathrm{x})$ and mean zero.

The statistical parameters on Y are:

$$
\hat{Y}=\int_{-\infty}^{\infty} y p_{y}(y) d y
$$

and

$$
\sigma_{Y}^{2}=\int_{-\infty}^{\infty}[y-\hat{Y}]^{2} p_{y}(y) d y
$$

The probability distribution is:

$$
p_{Y}(y)=\frac{p_{X}\left(x_{1}\right)}{\left|f^{\prime}\left(x_{1}\right)\right|}+\frac{p_{X}\left(x_{2}\right)}{\left|f^{\prime}\left(x_{2}\right)\right|}
$$

with

$$
f^{\prime}\left(x_{i}\right)=\left[\frac{d f(x)}{d x}\right]_{x=x_{i}}
$$

and $x_{i}$ roots of $y=f(x)$. With these conditions the statistical parameters on $Y$ are:

$$
\begin{aligned}
& \hat{Y}=c \sigma_{X}^{2}+a \\
& \sigma_{Y}^{2}=2 c^{2} \sigma_{X}^{4}+b^{2} \sigma_{X}^{2}
\end{aligned}
$$

For a desiderated RMS tolerance $\sigma_{\mathrm{Y}}$ the RMS jitter tolerance on parameter $\mathrm{X}$ is:

- if $\mathrm{c} \neq 0$

$$
\sigma_{X}^{2}=\frac{-b^{2}+\sqrt{b^{4}+8 c^{2} \sigma_{Y}^{2}}}{4 c^{2}}
$$

- if $\mathrm{c}=0$ (Y linearly depends on $\mathrm{X})$

$$
\sigma_{Y}^{2}=b^{2} \sigma_{X}^{2}
$$

\subsubsection{Jitter sensitivities and tolerance budget}

In this section we report the results on the beam jitter sensitivities, which have been used to form a tolerance budget. In paragraph 6.1.2.1 and in particular for medium 
bunch case M2 we describe the method used to form the tolerance budgets. For other cases M6, L2 and L4 we only report the sensitivity and tolerance tables.

\subsubsection{Medium bunch cases}

Table (6.2) lists sensitivities for RF phase, RF voltage and chicane bend power supplies for the different systems along the two stage compressor linac. Each sensitivities, independently, causes a $10 \%$ peak current increase, $0.1 \%$ relative mean energy increase and $150 \mathrm{fsec}$ final timing increase. In general the sensitivities are approximately linear apart when a linac is operated on crest or in particular cases as in the relative peak current $\Delta \mathrm{I} / \mathrm{I}$ versus gun timing $\Delta \mathrm{t}_{0}$ indicated in figure (6.2) (upper center). In table (6.2) the parabolic dependence is indicated in bold text.

Table (6.2) Individual RMS sensitivities $\mathrm{p}_{\text {sen }}$ for medium bunch case M2. Each cause $\Delta \mathrm{I} / \mathrm{I}_{0}=+10 \%$ peak current change or $\Delta \mathrm{E} / \mathrm{E}_{0}=+0.1 \%$ final relative energy change or $\Delta \mathrm{t}=$ $150 \mathrm{fsec}$ final timing change. Parabolic dependence in bold text.

\begin{tabular}{|l|c|c|c|c|c|}
\hline Parameters & Sy. & Unit & $\Delta \mathbf{I} / \mathbf{I}_{\mathbf{0}}=+\mathbf{1 0} \%$ & $\Delta \mathbf{E} / \mathbf{E}_{\mathbf{0}}=+\mathbf{0 . 1 \%}$ & $\Delta \mathbf{t = + 1 5 0 f s e c}$ \\
\hline \hline L1 RF phase & $\phi_{1}$ & S-band deg & -0.26 & -0.65 & -0.19 \\
LX RF phase & $\phi_{\mathrm{x}}$ & X-band deg & 0.92 & -3.99 & $\mathbf{1 0 . 7 0}$ \\
L2 RF phase & $\phi_{2}$ & S-band deg & -4.09 & 1.92 & -1.89 \\
L3 RF phase & $\phi_{3}$ & S-band deg & -2.39 & 1.13 & -1.11 \\
L4 RF phase & $\phi_{4}$ & S-band deg & $>10$ & 0.32 & $>10$ \\
\hline \hline L1 RF voltage & $\Delta \mathrm{V}_{1} / \mathrm{V}_{1}$ & $\%$ & -10.09 & -0.53 & 0.25 \\
LXRF voltage & $\Delta \mathrm{V}_{\mathrm{x}} / \mathrm{V}_{\mathrm{x}}$ & $\%$ & 8.61 & 5.62 & 2.28 \\
L2 RF voltage & $\Delta \mathrm{V}_{2} / \mathrm{V}_{2}$ & $\%$ & -7.47 & 1.30 & -1.20 \\
L3 RF voltage & $\Delta \mathrm{V}_{3} / \mathrm{V}_{3}$ & $\%$ & -4.34 & 0.76 & -0.71 \\
L4 RF voltage & $\Delta \mathrm{V}_{4} / \mathrm{V}_{4}$ & $\%$ & $>20$ & 0.20 & $>20$ \\
\hline \hline Gun timing & $\Delta \mathrm{t}_{0}$ & $p s e c$ & 3.16 & 0.47 & -2.52 \\
Initial charge & $\Delta \mathrm{Q}_{\mathrm{C}} \mathrm{Q}$ & $\%$ & -13.98 & -14.50 & 18.25 \\
\hline \hline BC1 chicane & $\Delta \mathrm{B}_{1} / \mathrm{B}_{1}$ & $\%$ & -1.07 & -0.25 & -0.22 \\
BC2 chicane & $\Delta \mathrm{B}_{2} / \mathrm{B}_{2}$ & $\%$ & -2.01 & -0.57 & -0.27 \\
\hline
\end{tabular}

In addition figure (6.2) plots rms final relative mean energy $\Delta \mathrm{E} / \mathrm{E}$ and $\mathrm{rms}$ final timing jitter $\Delta \mathrm{t}_{\mathrm{f}}$ versus gun timing jitters $\Delta \mathrm{t}_{0}$ (upper plots) and relative charge variations $\Delta \mathrm{Q} / \mathrm{Q}$ at the injector (lower plots). Upper left plot in figure (6.2) indicates that a $2.5 \mathrm{psec}$ gun timing jitters causes a 150 fsec final timing jitter while upper right plot in figure (6.2) indicates that a 0.47 psec gun timing causes a $0.1 \%$ relative electron beam energy jitter in the undulator. Another critical part, from sensitivity point of view, is linac 1 which sensitivities are reported in figure (6.3).

The sensitivities reported in table (6.2) are used to generate a tolerance budget based on summing random, uncorrelated effects [1]:

$$
\sqrt{\sum_{i=1}^{14}\left(\frac{p_{t o l}}{p_{\text {sen }}}\right)_{i}^{2}}<1
$$


The sensitivities $p_{\text {sen }}$ in table (6.2) are weighting values for the summation in (6.10). If the tolerance are chosen such that, $\mathrm{p}_{\text {tol }}<\mathrm{p}_{\text {sen }}$ for all $\mathrm{i}$, a budget is formed. Table (6.3) lists three possible tolerance budgets. If the first budget (fourth column in table (6.3)) is used, the relative peak current fluctuations at the linac end will be held to $<10 \% \mathrm{rms}$. Analogous considerations can be done for fifth and sixth columns in table (6.3). If the smaller tolerance from each column is applied (bold text), all three performance requirements $\left(|\Delta \mathrm{I} / \mathrm{I}|<10 \%,|\Delta \mathrm{E} / \mathrm{E}|<0.1 \%\right.$ and $\left|\Delta \mathrm{t}_{\mathrm{f}}\right|<150$ fsec $)$ will simultaneously be met.

We can see from table (6.3) that the relative mean energy jitter is the leading output parameter to fix the tolerance on photo-injector and linacs. Furthermore we want to point out that between voltage and phase of the accelerating section and photo-injector parameters there is a give-and-take, that is photo-injector parameters could be looser if voltage would be tighter and vice versa.

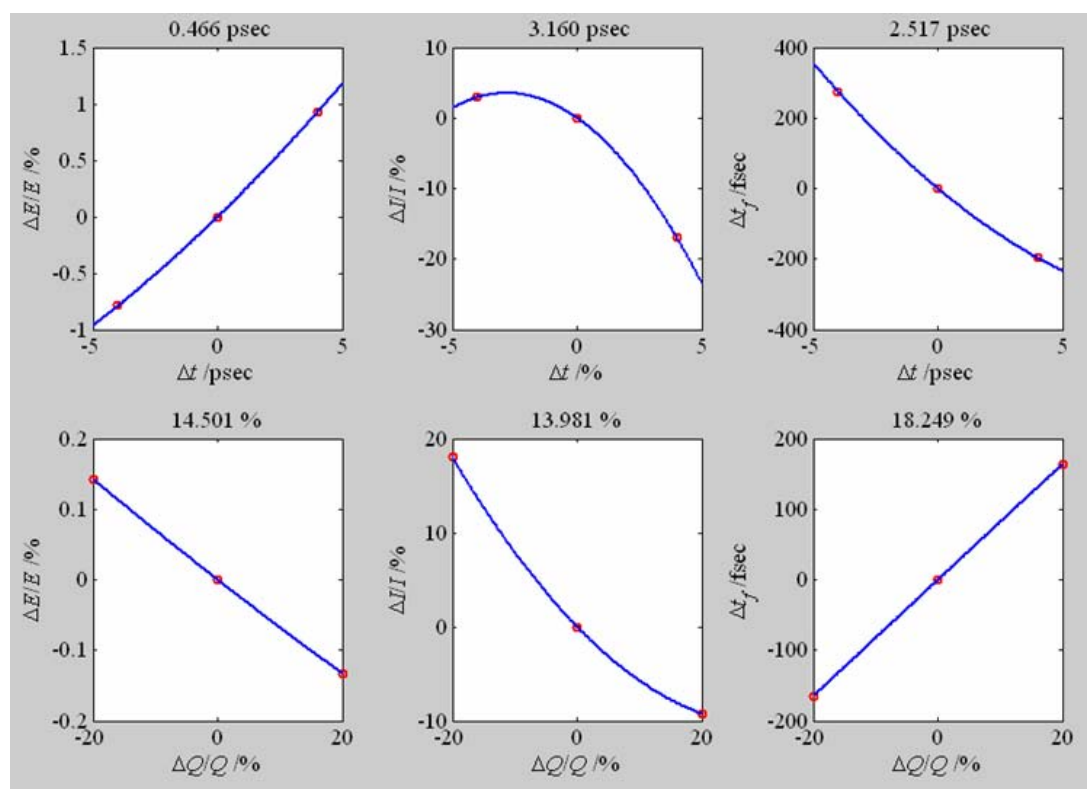

Figure (6.2) Rms final relative mean energy $\Delta \mathrm{E} / \mathrm{E}$, relative peak current $\Delta \mathrm{I} / \mathrm{I}$ and final timing jitter $\Delta \mathrm{t}_{\mathrm{f}}$ as a function of gun timing jitter $\Delta \mathrm{t}_{0}$ (upper plots) and relative initial bunch charge variations $\Delta \mathrm{Q} / \mathrm{Q}$ (lower plots). 

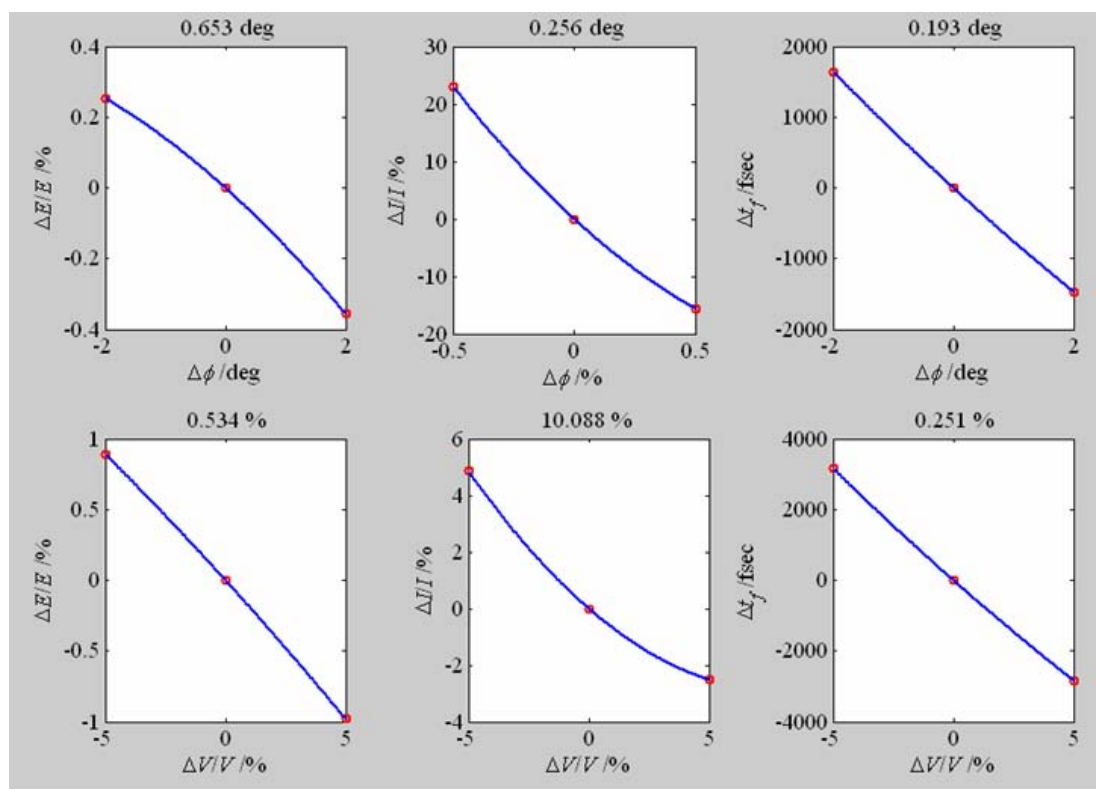

Figure (6.3) Rms final relative mean energy $\Delta \mathrm{E} / \mathrm{E}$, relative peak current $\Delta \mathrm{I} / \mathrm{I}$ and final timing jitter $\Delta \mathrm{t}_{\mathrm{f}}$ as a function of RF phase (upper plots) and RF amplitude (lower plots) in LINAC 1.

Table (6.3) Medium bunch case (M2) tolerance budget ( $\mathrm{p}_{\mathrm{tol}}$ ) for $<0.1 \%$ RMS final relative mean energy, $<10 \%$ RMS peak current jitter or $<150$ fsec final timing jitter. The tighter tolerance is in BOLD text and all criteria are satisfied if the tighter tolerance is applied.

\begin{tabular}{|l|c|c|c|c|c|}
\hline Parameters & Sy. & Unit & $\left|\Delta \mathbf{I} / \mathbf{I}_{\mathbf{0}}\right|=\mathbf{1 0} \%$ & $\left|\Delta \mathbf{E} / \mathbf{E}_{\mathbf{0}}\right|=\mathbf{0 . 1} \%$ & $|\Delta \mathbf{t}|=\mathbf{1 5 0 f \text { sec }}$ \\
\hline L1 RF phase & $\phi_{1}$ & S-band deg & 0.15 & 0.15 & $\mathbf{0 . 1 0}$ \\
LX RF phase & $\phi_{\mathrm{x}}$ & X-band deg & $\mathbf{0 . 5 0}$ & 0.60 & 0.70 \\
L2 RF phase & $\phi_{2}$ & S-band deg & 0.50 & $\mathbf{0 . 2 0}$ & 0.40 \\
L3 RF phase & $\phi_{3}$ & S-band deg & 0.20 & $\mathbf{0 . 1 5}$ & 0.20 \\
L4 RF phase & $\phi_{4}$ & S-band deg & 0.70 & $\mathbf{0 . 1 0}$ & 1.00 \\
\hline L1 RF volt. & $\Delta \mathrm{V}_{1} / \mathrm{V}_{1}$ & $\%$ & 0.70 & $\mathbf{0 . 1 5}$ & $\mathbf{0 . 1 5}$ \\
LXRF volt. & $\Delta \mathrm{V}_{\mathrm{x}} / \mathrm{V}_{\mathrm{x}}$ & $\%$ & 0.60 & 1.00 & $\mathbf{0 . 5 0}$ \\
L2 RF volt. & $\Delta \mathrm{V}_{2} / \mathrm{V}_{2}$ & $\%$ & 0.50 & $\mathbf{0 . 1 5}$ & 0.20 \\
L3 RF volt. & $\Delta \mathrm{V}_{3} / \mathrm{V}_{3}$ & $\%$ & 0.30 & $\mathbf{0 . 1 0}$ & 0.15 \\
L4 RF volt. & $\Delta \mathrm{V}_{4} / \mathrm{V}_{4}$ & $\%$ & 1.40 & $\mathbf{0 . 0 8}$ & 1.00 \\
\hline Gun timing & $\Delta \mathrm{t}_{0}$ & $p s e c$ & 0.35 & $\mathbf{0 . 3 0}$ & 0.50 \\
Initial charge & $\Delta \mathrm{Q}_{\mathrm{Q}} / \mathrm{Q}$ & $\%$ & 5.00 & $\mathbf{4 . 0 0}$ & 5.00 \\
\hline BC1 chicane & $\Delta \mathrm{B}_{1} / \mathrm{B}_{1}$ & $\%$ & 0.07 & 0.02 & $\mathbf{0 . 0 2}$ \\
BC2 chicane & $\Delta \mathrm{B}_{2} / \mathrm{B}_{2}$ & $\%$ & 0.14 & 0.04 & $\mathbf{0 . 0 2}$ \\
\hline
\end{tabular}


Table (6.4) collects the sensitivities for medium bunch case M6 with ramped current distribution out of photo-injector. The sensitivities of the relative peak current and final timing are approximately the same as case M2. The final relative mean energy become more sensible, up to factor 2 , to phase and amplitude of the linac 1, linac 2 and linac 3. This fact likely depends on different current distribution out of photo-injector because the phase and amplitude in linacs of case M6 are the same as linacs of case M2.

In addition we point out that in this case the final timing becomes more sensible to timing jitters out of photo-injector. In fact a 1.66 psec gun timing jitter causes a 150 fsec final timing jitter.

In the same manner as case M2 these sensitivities are used to form a tolerance budget and results are reported in table (6.5). Obviously seen the different sensitivities of the parameters with respect to final relative mean energy jitter between case M2 and M6 the tolerance budget for case M6 becomes more stringent to satisfy the requirement on final energy. In particular we point out that the tolerance on relative amplitude in linac 4 should be in the order of magnitude of $5 \cdot 10^{-4}$ and an initial timing jitter out of photoinjector of $250 \mathrm{fsec}$.

Table (6.4) Individual RMS sensitivities $\mathrm{p}_{\text {sen }}$ for medium bunch case M6. Each cause $\Delta \mathrm{I} / \mathrm{I}_{0}=+10 \%$ peak current change or $\Delta \mathrm{E} / \mathrm{E}_{0}=+0.1 \%$ final relative energy change or $\Delta \mathrm{t}=$ $150 \mathrm{fsec}$ final timing change. Parabolic dependence in bold text.

\begin{tabular}{|l|c|c|c|c|c|}
\hline Parameters & Sy. & Unit & $\Delta \mathbf{I} / \mathbf{I}_{\mathbf{0}}=+\mathbf{1 0} \%$ & $\Delta \mathbf{E} / \mathbf{E}_{\mathbf{0}}=+\mathbf{0 . 1 \%}$ & $\Delta \mathbf{t = + 1 5 0 f s e c}$ \\
\hline \hline L1 RF phase & $\phi_{1}$ & S-band deg & -0.26 & 0.27 & -0.18 \\
LX RF phase & $\phi_{\mathrm{x}}$ & X-band deg & 0.85 & -3.35 & $\mathbf{9 . 8 1}$ \\
L2 RF phase & $\phi_{2}$ & S-band deg & -4.24 & 0.96 & -1.87 \\
L3 RF phase & $\phi_{3}$ & S-band deg & -2.48 & 0.56 & -1.10 \\
L4 RF phase & $\phi_{4}$ & S-band deg & $>10$ & -0.33 & $>10$ \\
\hline \hline L1 RF voltage & $\Delta \mathrm{V}_{1} / \mathrm{V}_{1}$ & $\%$ & $\mathbf{1 3 . 4 2}$ & 0.48 & -0.24 \\
LXRF voltage & $\Delta \mathrm{V}_{\mathrm{x}} / \mathrm{V}_{\mathrm{x}}$ & $\%$ & 11.05 & -3.85 & 2.06 \\
L2 RF voltage & $\Delta \mathrm{V}_{2} / \mathrm{V}_{2}$ & $\%$ & -7.91 & 0.63 & -1.19 \\
L3 RF voltage & $\Delta \mathrm{V}_{3} / \mathrm{V}_{3}$ & $\%$ & -4.60 & 0.37 & -0.70 \\
L4 RF voltage & $\Delta \mathrm{V}_{4} / \mathrm{V}_{4}$ & $\%$ & $>20$ & 0.20 & $>20$ \\
\hline \hline Gun timing & $\Delta \mathrm{t}_{0}$ & $p s e c$ & 3.31 & 0.42 & -1.66 \\
Initial charge & $\Delta \mathrm{Q} / \mathrm{Q}$ & $\%$ & -35.73 & -8.31 & 18.67 \\
\hline \hline BC1 chicane & $\Delta \mathrm{B}_{1} / \mathrm{B}_{1}$ & $\%$ & -1.56 & 1.59 & -0.20 \\
BC2 chicane & $\Delta \mathrm{B}_{2} / \mathrm{B}_{2}$ & $\%$ & -2.22 & 0.52 & -0.27 \\
\hline
\end{tabular}

\subsubsection{Long bunch cases}

Table (6.6) and table (6.8) collect sensitivities, respectively, for case L2 and L4. Case L4 is with ramped current distribution out of photo-injector. As in previous paragraph these sensitivities have been used to form tolerance budgets for case L2 and L4 reported, respectively, in table (6.70 and table (6.9). 
Table (6.5) Medium bunch case M6 tolerance budget $\left(\mathrm{p}_{\text {tol }}\right)$ for $<0.1 \%$ RMS final relative mean energy, $<10 \%$ RMS peak current jitter or $<150$ fsec final timing jitter. The tighter tolerance is in BOLD text and all criteria are satisfied if the tighter tolerance is applied.

\begin{tabular}{|l|c|c|c|c|c|}
\hline Parameters & Sy. & Unit & $\left|\Delta \mathbf{I} / \mathbf{I}_{\mathbf{0}}\right|=\mathbf{1 0} \%$ & $\left|\Delta \mathbf{E} / \mathbf{E}_{\mathbf{0}}\right|=\mathbf{0 . 1 \%}$ & $|\Delta \mathbf{t}|=\mathbf{1 5 0 f s e c}$ \\
\hline \hline L1 RF phase & $\phi_{1}$ & S-band deg & 0.20 & $\mathbf{0 . 1 0}$ & $\mathbf{0 . 1 0}$ \\
LX RF phase & $\phi_{\mathrm{x}}$ & X-band deg & 0.50 & $\mathbf{0 . 3 0}$ & 0.70 \\
L2 RF phase & $\phi_{2}$ & S-band deg & 0.50 & $\mathbf{0 . 1 0}$ & 0.40 \\
L3 RF phase & $\phi_{3}$ & S-band deg & 0.20 & $\mathbf{0 . 1 0}$ & 0.20 \\
L4 RF phase & $\phi_{4}$ & S-band deg & 0.70 & $\mathbf{0 . 1 0}$ & 1.00 \\
\hline \hline L1 RF volt. & $\Delta \mathrm{V}_{1} / \mathrm{V}_{1}$ & $\%$ & 1.00 & $\mathbf{0 . 1 0}$ & 0.15 \\
LXRF volt. & $\Delta \mathrm{V}_{\mathrm{x}} / \mathrm{V}_{\mathrm{x}}$ & $\%$ & 0.80 & $\mathbf{0 . 5 0}$ & $\mathbf{0 . 5 0}$ \\
L2 RF volt. & $\Delta \mathrm{V}_{2} / \mathrm{V}_{2}$ & $\%$ & 0.80 & $\mathbf{0 . 1 0}$ & 0.20 \\
L3 RF volt. & $\Delta \mathrm{V}_{3} / \mathrm{V}_{3}$ & $\%$ & 0.50 & $\mathbf{0 . 1 0}$ & 0.15 \\
L4 RF volt. & $\Delta \mathrm{V}_{4} / \mathrm{V}_{4}$ & $\%$ & 1.50 & $\mathbf{0 . 0 5}$ & 1.00 \\
\hline \hline Gun timing & $\Delta \mathrm{t}_{0}$ & $p s e c$ & 0.35 & $\mathbf{0 . 2 5}$ & 0.35 \\
Initial charge & $\Delta \mathrm{Q} / \mathrm{Q}$ & $\%$ & 5.00 & $\mathbf{3 . 0 0}$ & 4.00 \\
\hline \hline BC1 chicane & $\Delta \mathrm{B}_{1} / \mathrm{B}_{1}$ & $\%$ & 0.15 & 0.10 & $\mathbf{0 . 0 2}$ \\
BC2 chicane & $\Delta \mathrm{B}_{2} / \mathrm{B}_{2}$ & $\%$ & 0.25 & 0.03 & $\mathbf{0 . 0 2}$ \\
\hline
\end{tabular}

Table (6.6) Individual RMS sensitivities $\mathrm{p}_{\text {sen }}$ for long bunch case L2. Each cause $\Delta \mathrm{I} / \mathrm{I}_{0}=+10 \%$ peak current change or $\Delta \mathrm{E} / \mathrm{E}_{0}=+0.1 \%$ final relative energy change or $\Delta \mathrm{t}=$ $150 \mathrm{fsec}$ final timing change. In Linac 4 is operated on crest and a phase variation can only decrease the final energy. Parabolic dependence in bold text.

\begin{tabular}{|l|c|c|c|c|c|}
\hline Parameters & Sy. & Unit & $\Delta \mathbf{I} / \mathbf{I}_{\mathbf{0}}=+\mathbf{1 0} \%$ & $\Delta \mathbf{E} / \mathbf{E}_{\mathbf{0}}=+\mathbf{0 . 1 \%}$ & $\Delta \mathbf{t = + 1 5 0 f s e c}$ \\
\hline \hline L1 RF phase & $\phi_{1}$ & S-band deg & -0.71 & 0.81 & -0.15 \\
LX RF phase & $\phi_{\mathrm{x}}$ & X-band deg & 2.93 & -5.57 & 1.73 \\
L2 RF phase & $\phi_{2}$ & S-band deg & -3.36 & 3.58 & -3.11 \\
L3 RF phase & $\phi_{3}$ & S-band deg & -1.77 & 0.94 & -0.67 \\
L4 RF phase & $\phi_{4}$ & S-band deg & $>10$ & $\mathbf{3 . 0 3}$ & $>10$ \\
\hline L1 RF voltage & $\Delta \mathrm{V}_{1} / \mathrm{V}_{1}$ & $\%$ & 1.53 & 2.21 & -0.21 \\
LXRF voltage & $\Delta \mathrm{V}_{\mathrm{x}} / \mathrm{V}_{\mathrm{x}}$ & $\%$ & -4.76 & $\mathbf{2 5 . 7 7}$ & 3.31 \\
L2 RF voltage & $\Delta \mathrm{V}_{2} / \mathrm{V}_{2}$ & $\%$ & -7.66 & 0.82 & -0.52 \\
L3 RF voltage & $\Delta \mathrm{V}_{3} / \mathrm{V}_{3}$ & $\%$ & -6.63 & 0.50 & -0.31 \\
L4 RF voltage & $\Delta \mathrm{V}_{4} / \mathrm{V}_{4}$ & $\%$ & $>20$ & 0.20 & $>20$ \\
\hline Gun timing & $\Delta \mathrm{t}_{0}$ & $p s e c$ & -1.17 & 0.51 & $\mathbf{2 . 9 4}$ \\
Initial charge & $\Delta \mathrm{Q}_{\mathrm{C}} \mathrm{Q}$ & $\%$ & $>40$ & 10.56 & 10.16 \\
\hline \hline BC1 chicane & $\Delta \mathrm{B}_{1} / \mathrm{B}_{1}$ & $\%$ & 1.72 & -0.85 & -0.23 \\
BC2 chicane & $\Delta \mathrm{B}_{2} / \mathrm{B}_{2}$ & $\%$ & -2.58 & $\mathbf{2 . 2 2}$ & -0.12 \\
\hline
\end{tabular}


From tables (6.7) and (6.9) we can see that the relative mean energy jitter and final timing jitter are the leading output parameter to fix the tolerance on photo-injector and linacs in both cases. The principal different between tolerance budgets in case L2 and L4 is in initial bunch charge variations and relative voltage of the X-band. In fact tolerance budget for case L2 satisfies requirement on final timing jitter if fluctuations of relative amplitude of X-band and initial bunch variations are, respectively, held less than $0.30 \%$ rms and 3\% rms in the summation 6.10. These values are needed because in the case L2 final timing jitter is more sensible to phase of linac 1 than in the case L4. The sensitivity is 1.6 times more stringent in case L2 than in case L4. How seen in previous medium case tolerances on initial charge variations and timing jitter out of photo-injector could become looser if, in general, tolerances on parameters of the linacs, such as phase and amplitude of linac 1, will be tighter.

Table (6.7) Long bunch case L2 tolerance budget $\left(\mathrm{p}_{\text {tol }}\right)$ for $<0.1 \%$ RMS final relative mean energy, $<10 \%$ RMS peak current jitter or $<150 \mathrm{fsec}$ final timing jitter. The tighter tolerance is in BOLD text and all criteria are satisfied if the tighter tolerance is applied.

\begin{tabular}{|l|c|c|c|c|c|}
\hline Parameters & Sy. & Unit & $\left|\Delta \mathbf{I} / \mathbf{I}_{\mathbf{0}}\right|=\mathbf{1 0} \%$ & $\left|\Delta \mathbf{E} / \mathbf{E}_{\mathbf{0}}\right|=\mathbf{0 . 1} \%$ & $|\Delta \mathbf{t}|=\mathbf{1 5 0 f s e c}$ \\
\hline \hline L1 RF phase & $\phi_{1}$ & S-band deg & 0.30 & 0.20 & $\mathbf{0 . 0 9}$ \\
LX RF phase & $\phi_{\mathrm{x}}$ & X-band deg & 0.70 & $\mathbf{0 . 5 0}$ & $\mathbf{0 . 5 0}$ \\
L2 RF phase & $\phi_{2}$ & S-band deg & 0.50 & 0.40 & $\mathbf{0 . 2 0}$ \\
L3 RF phase & $\phi_{3}$ & S-band deg & 0.25 & $\mathbf{0 . 1 0}$ & $\mathbf{0 . 1 0}$ \\
L4 RF phase & $\phi_{4}$ & S-band deg & 1.50 & $\mathbf{0 . 2 5}$ & 0.60 \\
\hline L1 RF volt. & $\Delta \mathrm{V}_{1} / \mathrm{V}_{1}$ & $\%$ & 0.30 & 0.25 & $\mathbf{0 . 1 0}$ \\
LXRF volt. & $\Delta \mathrm{V}_{\mathrm{x}} / \mathrm{V}_{\mathrm{x}}$ & $\%$ & 0.60 & 1.90 & $\mathbf{0 . 3 0}$ \\
L2 RF volt. & $\Delta \mathrm{V}_{2} / \mathrm{V}_{2}$ & $\%$ & 1.00 & $\mathbf{0 . 1 0}$ & $\mathbf{0 . 1 0}$ \\
L3 RF volt. & $\Delta \mathrm{V}_{3} / \mathrm{V}_{3}$ & $\%$ & 0.90 & $\mathbf{0 . 1 0}$ & $\mathbf{0 . 1 0}$ \\
L4 RF volt. & $\Delta \mathrm{V}_{4} / \mathrm{V}_{4}$ & $\%$ & 2.50 & $\mathbf{0 . 0 8}$ & 1.20 \\
\hline \hline Gun timing & $\Delta \mathrm{t}_{0}$ & $p s e c$ & 0.80 & $\mathbf{0 . 3 5}$ & 0.40 \\
Initial charge & $\Delta \mathrm{Q} / \mathrm{Q}$ & $\%$ & 10.00 & 4.00 & $\mathbf{3 . 0 0}$ \\
\hline \hline BC1 chicane & $\Delta \mathrm{B}_{1} / \mathrm{B}_{1}$ & $\%$ & 0.15 & 0.06 & $\mathbf{0 . 0 2}$ \\
BC2 chicane & $\Delta \mathrm{B}_{2} / \mathrm{B}_{2}$ & $\%$ & 0.20 & 0.15 & $\mathbf{0 . 0 1}$ \\
\hline
\end{tabular}


Table (6.8) Individual RMS sensitivities $p_{\text {sen }}$ for long bunch case L4. Each cause $\Delta \mathrm{I} / \mathrm{I}_{0}=+10 \%$ peak current change or $\Delta \mathrm{E} / \mathrm{E}_{0}=+0.1 \%$ final relative energy change or $\Delta \mathrm{t}=$ 150fsec final timing change. Parabolic dependence in bold text.

\begin{tabular}{|l|c|c|c|c|c|}
\hline Parameters & Sy. & Unit & $\Delta \mathbf{I} / \mathbf{I}_{\mathbf{0}}=+\mathbf{1 0} \%$ & $\Delta \mathbf{E} / \mathbf{E}_{\mathbf{0}}=+\mathbf{0 . 1} \%$ & $\Delta \mathbf{t}=+\mathbf{1 5 0}$ fsec \\
\hline L1 RF phase & $\phi_{1}$ & S-band deg & -0.52 & 0.87 & -0.25 \\
LX RF phase & $\phi_{\mathrm{x}}$ & X-band deg & 1.30 & -4.42 & $\mathbf{9 . 7 9}$ \\
L2 RF phase & $\phi_{2}$ & S-band deg & -2.23 & 1.16 & -1.06 \\
L3 RF phase & $\phi_{3}$ & S-band deg & -1.29 & 0.68 & -0.62 \\
L4 RF phase & $\phi_{4}$ & S-band deg & $>10$ & -1.36 & $>10$ \\
\hline L1 RF voltage & $\Delta \mathrm{V}_{1} / \mathrm{V}_{1}$ & $\%$ & 1.32 & 1.59 & -0.21 \\
LXRF voltage & $\Delta \mathrm{V}_{\mathrm{x}} / \mathrm{V}_{\mathrm{x}}$ & $\%$ & -25.15 & -13.90 & 2.04 \\
L2 RF voltage & $\Delta \mathrm{V}_{2} / \mathrm{V}_{2}$ & $\%$ & -7.25 & 0.74 & -0.60 \\
L3 RF voltage & $\Delta \mathrm{V}_{3} / \mathrm{V}_{3}$ & $\%$ & -4.21 & 0.43 & -0.35 \\
L4 RF voltage & $\Delta \mathrm{V}_{4} / \mathrm{V}_{4}$ & $\%$ & $>20$ & 0.20 & $>20$ \\
\hline Gun timing & $\Delta \mathrm{t}_{0}$ & $p s e c$ & 8.70 & 0.50 & -4.44 \\
Initial charge & $\Delta \mathrm{Q} / \mathrm{Q}$ & $\%$ & -20.65 & -9.75 & 11.69 \\
\hline \hline BC1 chicane & $\Delta \mathrm{B}_{1} / \mathrm{B}_{1}$ & $\%$ & 1.17 & -0.64 & -0.26 \\
BC2 chicane & $\Delta \mathrm{B}_{2} / \mathrm{B}_{2}$ & $\%$ & -1.67 & 0.95 & -0.13 \\
\hline
\end{tabular}

Table (6.9) Long bunch case L4 tolerance budget ( $\mathrm{p}_{\text {tol }}$ ) for $<0.1 \%$ RMS final relative mean energy, $<10 \%$ RMS peak current jitter or $<150$ fsec final timing jitter. The tighter tolerance is in BOLD text and all criteria are satisfied if the tighter tolerance is applied.

\begin{tabular}{|l|c|c|c|c|c|}
\hline Parameters & Sy. & Unit & $\left|\Delta \mathbf{I} / \mathbf{I}_{\mathbf{0}}\right|=\mathbf{1 0 \%}$ & $\left|\Delta \mathbf{E} / \mathbf{E}_{\mathbf{0}}\right|=\mathbf{0 . 1 \%}$ & $|\Delta \mathbf{t}|=\mathbf{1 5 0 f s e c}$ \\
\hline L1 RF phase & $\phi_{1}$ & S-band deg & 0.30 & 0.12 & $\mathbf{0 . 1 0}$ \\
LX RF phase & $\phi_{\mathrm{x}}$ & X-band deg & 0.70 & $\mathbf{0 . 5 0}$ & 0.70 \\
L2 RF phase & $\phi_{2}$ & S-band deg & 0.50 & $\mathbf{0 . 1 5}$ & 0.25 \\
L3 RF phase & $\phi_{3}$ & S-band deg & 0.25 & $\mathbf{0 . 1 0}$ & 0.15 \\
L4 RF phase & $\phi_{4}$ & S-band deg & 1.50 & $\mathbf{0 . 1 5}$ & 1.00 \\
\hline L1 RF volt. & $\Delta \mathrm{V}_{1} / \mathrm{V}_{1}$ & $\%$ & 0.30 & 0.15 & $\mathbf{0 . 1 0}$ \\
LXRF volt. & $\Delta \mathrm{V}_{\mathrm{x}} / \mathrm{V}_{\mathrm{x}}$ & $\%$ & 2.00 & 0.90 & $\mathbf{0 . 6 0}$ \\
L2 RF volt. & $\Delta \mathrm{V}_{2} / \mathrm{V}_{2}$ & $\%$ & 1.60 & $\mathbf{0 . 1 5}$ & $\mathbf{0 . 1 5}$ \\
L3 RF volt. & $\Delta \mathrm{V}_{3} / \mathrm{V}_{3}$ & $\%$ & 0.90 & $\mathbf{0 . 1 0}$ & $\mathbf{0 . 1 0}$ \\
L4 RF volt. & $\Delta \mathrm{V}_{4} / \mathrm{V}_{4}$ & $\%$ & 2.00 & $\mathbf{0 . 0 8}$ & 1.20 \\
\hline Gun timing & $\Delta \mathrm{t}_{0}$ & $p s e c$ & 1.00 & $\mathbf{0 . 3 5}$ & 0.60 \\
Initial charge & $\Delta \mathrm{Q} / \mathrm{Q}$ & $\%$ & 6.00 & $\mathbf{4 . 0 0}$ & 5.00 \\
\hline BC1 chicane & $\Delta \mathrm{B}_{1} / \mathrm{B}_{1}$ & $\%$ & 0.08 & 0.04 & $\mathbf{0 . 0 2}$ \\
BC2 chicane & $\Delta \mathrm{B}_{2} / \mathrm{B}_{2}$ & $\%$ & 0.10 & 0.06 & $\mathbf{0 . 0 2}$ \\
\hline
\end{tabular}




\subsubsection{Global jitter studies}

Tolerance budget has been adopted as RMS value for input parameters, such as phase and voltage in accelerating sections and R56 in chicanes, for Latin Hypercube Sampling (LHS). In this way it has been possible to get randomly sets of jitter inputs to run LiTrack in several situations of error of the linac. Statistical analysis on global output parameters as mean energy, peak current and final timing have confirmed the tolerance budget. Further statistical analyses have been done on various number of slice inside the bunch with a fixed slice length of $10 \mathrm{fsec}$.

Latin Hypercube Sampling (LHS) method is here described as reported in [52]. LHS method is a stratified random procedure, provides an efficient way of sampling variables from their distributions [53]. The LHS involves sampling $n s$ values from the prescribed distribution of each of $\mathrm{k}$ variables $\mathrm{X} 1, \mathrm{X} 2, \ldots \mathrm{Xk}$. The cumulative distribution for each variable is divided into $\mathrm{N}$ equiprobable intervals. A value is selected randomly from each interval. The $\mathrm{N}$ values obtained for each variable are paired randomly with the other variables. Unlike simple random sampling, this method ensures a full coverage of the range of each variable by maximally stratifying each marginal distribution.

The LHS can be summarized as:

- divide the cumulative distribution of each variable into $\mathrm{N}$ equiprobable intervals;

- from each interval select a value randomly, for the ith interval, the sampled cumulative probability can be written as [54]: $\operatorname{Prob}_{i}=(1=N) \cdot r_{u}+(i-1) / N$ where $r_{u}$ is uniformly distributed random number ranging from 0 to 1 ;

- transform the probability values sampled into the value $\mathrm{x}$ using the inverse of the distribution function $\mathrm{F}^{-1}: \mathrm{x}=\mathrm{F}^{-1}$ (Prob);

- the $\mathrm{N}$ values obtained for each variable $\mathrm{x}$ are paired randomly (equally likely combinations) with the ns values of the other variables.

\subsubsection{Medium bunch cases}

Figures (6.4) shows statistical results on 400 seeds, that is on 400 different configurations of the accelerations, compressions and photo-injector parameters for case M2 (upper plots) and M6 (lower plots).

From figure (6.4) we can see that for case L4 when tolerance budget in table (6.9) is applied to global jitter analysis the requirements on relative peak current and timing jitters at the linac end are respected better than in the case L2 (in particular on peak current). 

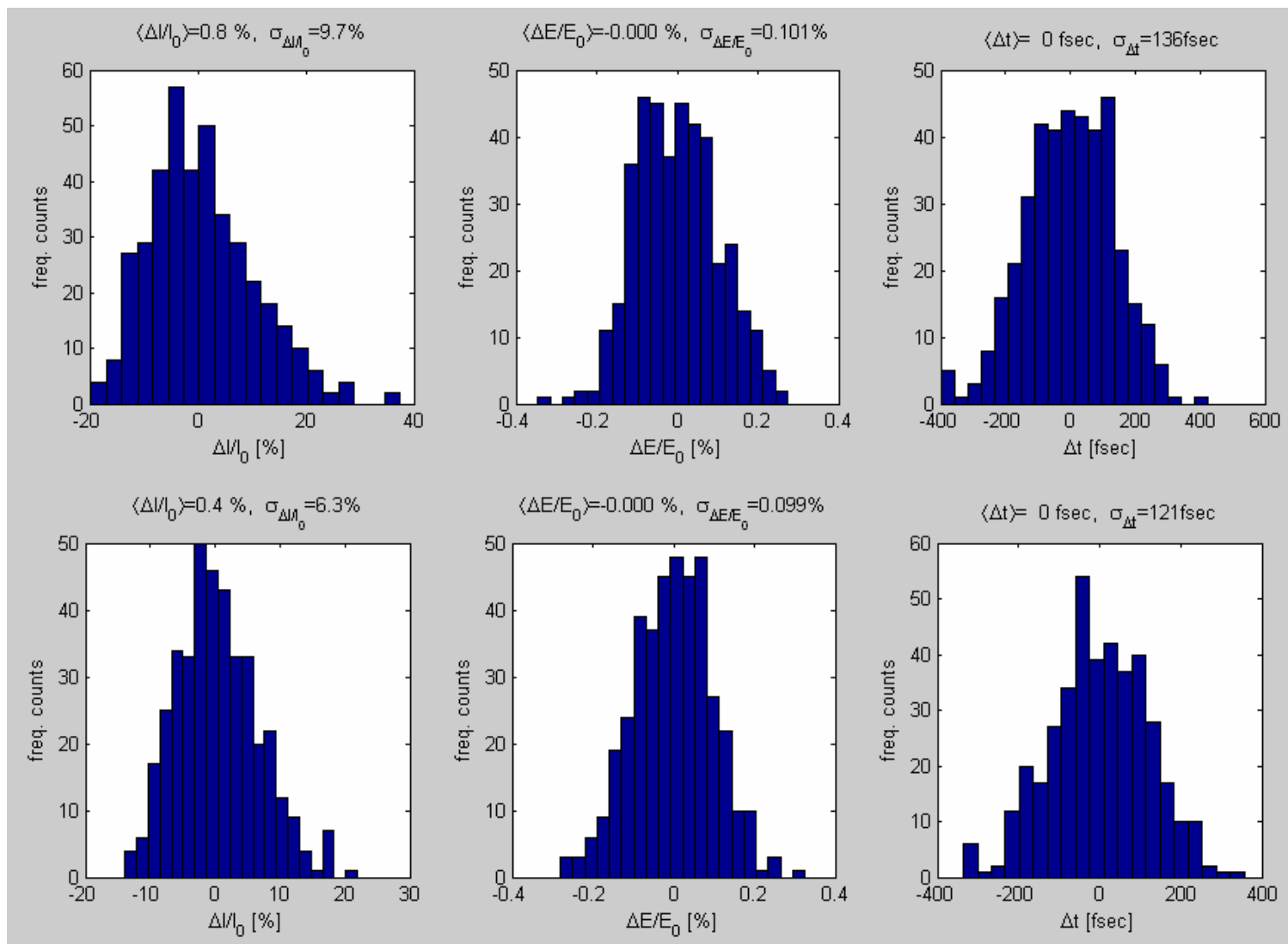

Figure (6.4) Histograms for medium bunch case M2 (upper plots) and M6 (lower plots) of the relative peak current $\Delta \mathrm{I} / \mathrm{I}_{0}$ (left), final relative mean energy $\Delta \mathrm{E} / \mathrm{E}_{0}$ (center) and final timing jitter $\Delta \mathrm{t}_{\mathrm{f}}$ (right). $\mathrm{I}_{0}$ is the nominal peak current and $\mathrm{E}_{0}$ is the nominal mean energy.

\subsubsection{Long bunch cases}

Figures (6.4) shows statistical results on 400 seeds, that is on 400 different configurations of the accelerations, compressions and photo-injector parameters for case M2 (upper plots) and M6 (lower plots).

In long bunch case global jitter analysis shows that when tolerance budget in tables (6.7) and (6.9) are, respectively, applied to case L2 and L4 the requirements on output parameters at the linac end are respected better in case L2 than in case L4. 

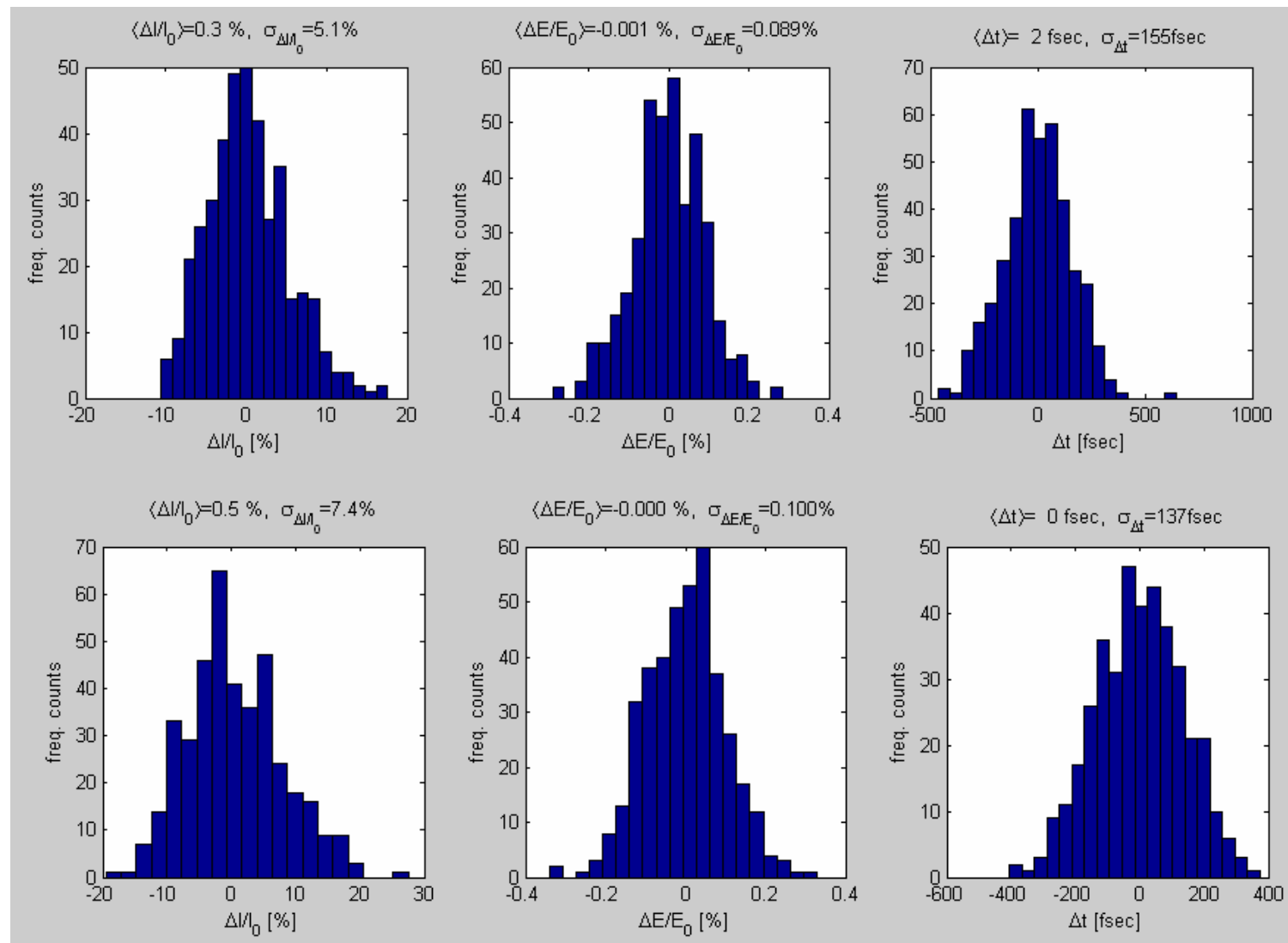

Figure (6.5) Histograms for long bunch case L2 (upper plots) and L4 (lower plots) of the relative peak current $\Delta \mathrm{I} / \mathrm{I}_{0}$ (left), final relative mean energy $\Delta \mathrm{E} / \mathrm{E}_{0}$ (center) and final timing jitter $\Delta \mathrm{t}_{\mathrm{f}}$ (right). $\mathrm{I}_{0}$ is the nominal peak current and $\mathrm{E}_{0}$ is the nominal mean energy.

\subsubsection{Slice jitter studies on longitudinal phase space}

Another requirement for particle distribution at the linac end is to have a flat longitudinal phase space. As previously explained we performed slice jitter studies (400 seeds for each case) and for each seed the energy variation in the central part of the bunch have been described by following function:

$$
E(t)=a_{0}\left(1 \pm \frac{\sigma_{a_{0}}}{a_{0}}\right)+a_{1}\left(1 \pm \frac{\sigma_{a_{1}}}{a_{1}}\right) \cdot t+a_{2}\left(1 \pm \frac{\sigma_{a_{2}}}{a_{2}}\right) \cdot t^{2}
$$

The flatness of the longitudinal phase space can be defined as the average quadratic component in the energy chirp $\left(\hat{a}_{2}\right)$ and rms fluctuation of this number $\left(\sigma_{a_{2}}\right)$.

\subsubsection{Medium bunch cases}

Table (6.10) collects statistical parameters of the polynomial coefficients of eq. (6.11) that fit the E-t data, $\mathrm{E}\left(\mathrm{t}_{\mathrm{i}}\right)$ to $\mathrm{E}_{\mathrm{i}}$, in a least squares sense. M2 and M6 cases are 
reported and we can see that quadratic energy chirp is 1.8 times less in M6 than in M2 while relative rms fluctuations are similar in both cases.

Figure (6.6) shows set of 10 randomly chosen seeds for medium bunch case M2 (a) and M6 (b) (left plots) together with relative first derivative of the energy with respect to time inside the bunch with quadratic chirp (central plots) and with quadratic chirp removed (right plots). In medium case M2, using a seed laser with a linear frequency chirp will allow compensating for a frequency chirp due to a quadratic energy variation in the electron beam while in case M6 quadratic chirp has been reduced using a ramped current distribution out of photo-injector.

Table (6.10) Statistical parameters of the polynomial coefficients for medium bunch case M2 and M6.

\begin{tabular}{|c|c|c|c|c|}
\hline \multicolumn{5}{|c|}{ Medium bunch case M2 } \\
\hline & Unit & mean & rms & rms/mean \% \\
\hline $\mathbf{a}_{\mathbf{0}}$ & $\mathrm{MeV}$ & 1138.24 & 1.183 & 0.10 \\
\hline $\mathbf{a}_{1}$ & $\mathrm{MeV} / \mathrm{psec}$ & 2.170 & 2.690 & 123.96 \\
\hline $\mathbf{a}_{2}$ & $\mathrm{MeV} / \mathrm{psec}^{2}$ & 6.450 & 2.210 & 34.26 \\
\hline \multicolumn{5}{|c|}{ Medium bunch case M6 } \\
\hline & Unit & mean & rms & rms/mean \% \\
\hline $\mathbf{a}_{0}$ & $\mathrm{MeV}$ & 1140.86 & 1.140 & 0.10 \\
\hline $\mathbf{a}_{1}$ & $\mathrm{MeV} / \mathrm{psec}$ & 0.0138 & 1.410 & 10220.94 \\
\hline $\mathbf{a}_{2}$ & $\mathrm{MeV} / \mathrm{psec}^{2}$ & 3.570 & 1.030 & 28.95 \\
\hline
\end{tabular}

\subsubsection{Long bunch cases}

As in previous medium case Table (6.11) collects statistical parameters of the polynomial coefficients of eq. (6.11) that fit the E-t data, $E\left(t_{i}\right)$ to $E_{i}$, in a least squares sense. $L 2$ and L4 cases are reported and we can see that quadratic energy chirp is 2.5 times less in L4 than in L2 while relative rms fluctuations are different in the two cases, respectively, $12.5 \%$ in $\mathrm{L} 2$ and $48.5 \%$ in L4.

Figure (6.7) shows set of 10 randomly chosen seeds for long bunch case L2 (a) and L4 (b) (left plots) together with relative first derivative of the energy with respect to time inside the bunch with quadratic chirp (central plots) and with quadratic chirp removed (right plots). As in the medium case M2, using a seed laser with a linear frequency chirp will allow compensating for a frequency chirp due to a quadratic energy variation in the electron beam for long case L2. From figure (6.7) we can see that, as in the medium case M6 even in the long bunch L4 quadratic chirp has been reduced using a ramped current distribution out of photo-injector as we can see from figure (6.7). 

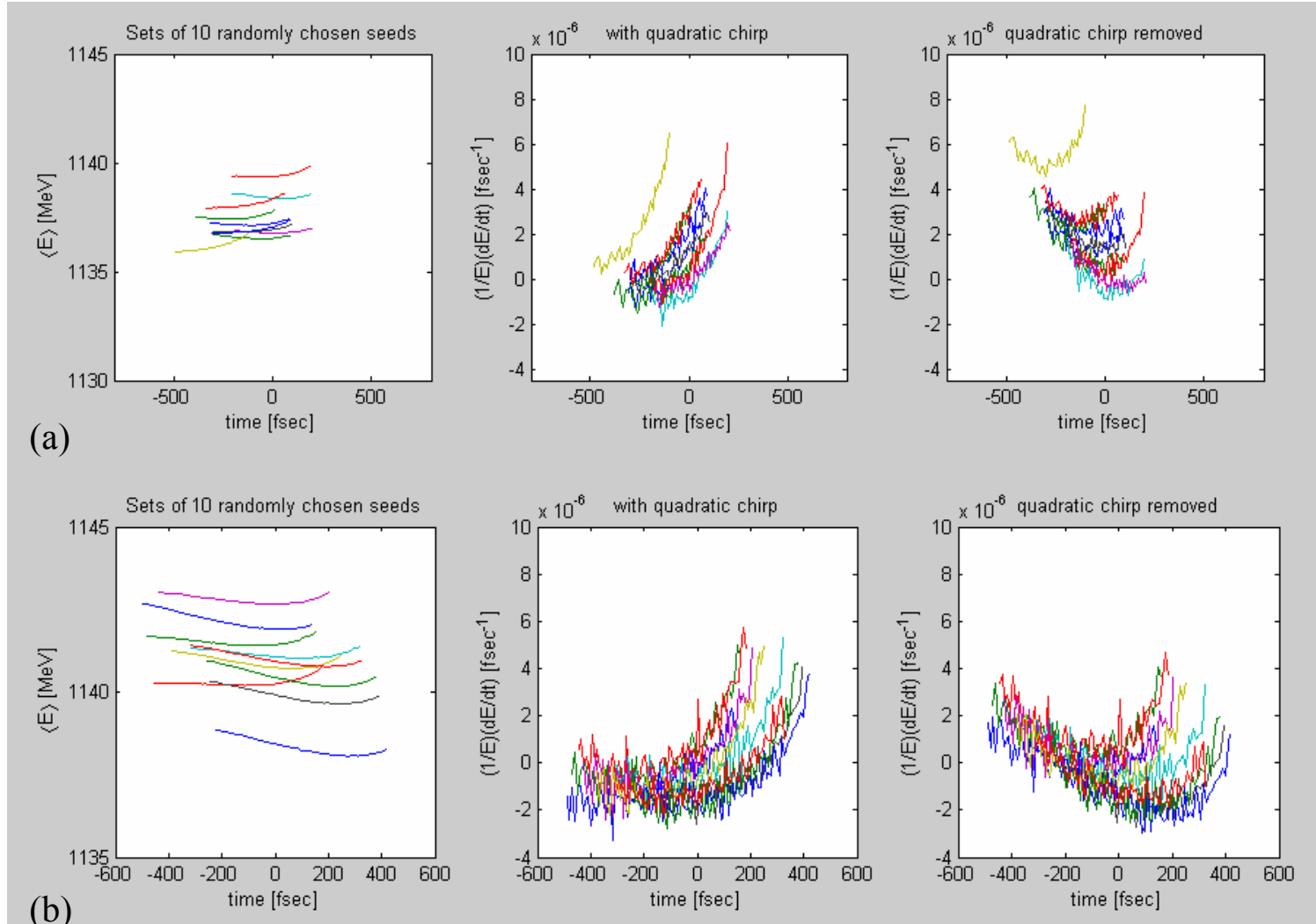

Figure (6.6) Set of 10 randomly chosen seeds for medium bunch case M2 (a) and M6 (b) (left plots) together with relative first derivative of the energy with respect to time inside the bunch with quadratic chirp (central plots) and with quadratic chirp removed (right plots).

Table (6.11) Statistical parameters of the polynomial coefficients for long bunch case L2 and L4.

\begin{tabular}{|c|c|c|c|c|}
\hline \multicolumn{5}{|c|}{ Long bunch case L2 } \\
\hline & Unit & mean & rms & rms/mean \% \\
\hline $\mathbf{a}_{\mathbf{0}}$ & $\mathrm{MeV}$ & 1188.00 & 1.040 & 0.09 \\
\hline $\mathbf{a}_{1}$ & $\mathrm{MeV} / \mathrm{psec}$ & -0.356 & 1.010 & -285.01 \\
\hline $\mathbf{a}_{2}$ & $\mathrm{MeV} / \mathrm{psec}^{2}$ & 2.530 & 0.317 & 12.50 \\
\hline \multicolumn{5}{|c|}{ Long bunch case L4 } \\
\hline & Unit & mean & rms & rms/mean \% \\
\hline $\mathbf{a}_{\mathbf{0}}$ & $\mathrm{MeV}$ & 1192.62 & 1.22 & 0.10 \\
\hline $\mathbf{a}_{1}$ & $\mathrm{MeV} / \mathrm{psec}$ & 0.446 & 0.828 & 185.67 \\
\hline $\mathbf{a}_{2}$ & $\mathrm{MeV} / \mathrm{psec}^{2}$ & 1.010 & 0.492 & 48.50 \\
\hline
\end{tabular}



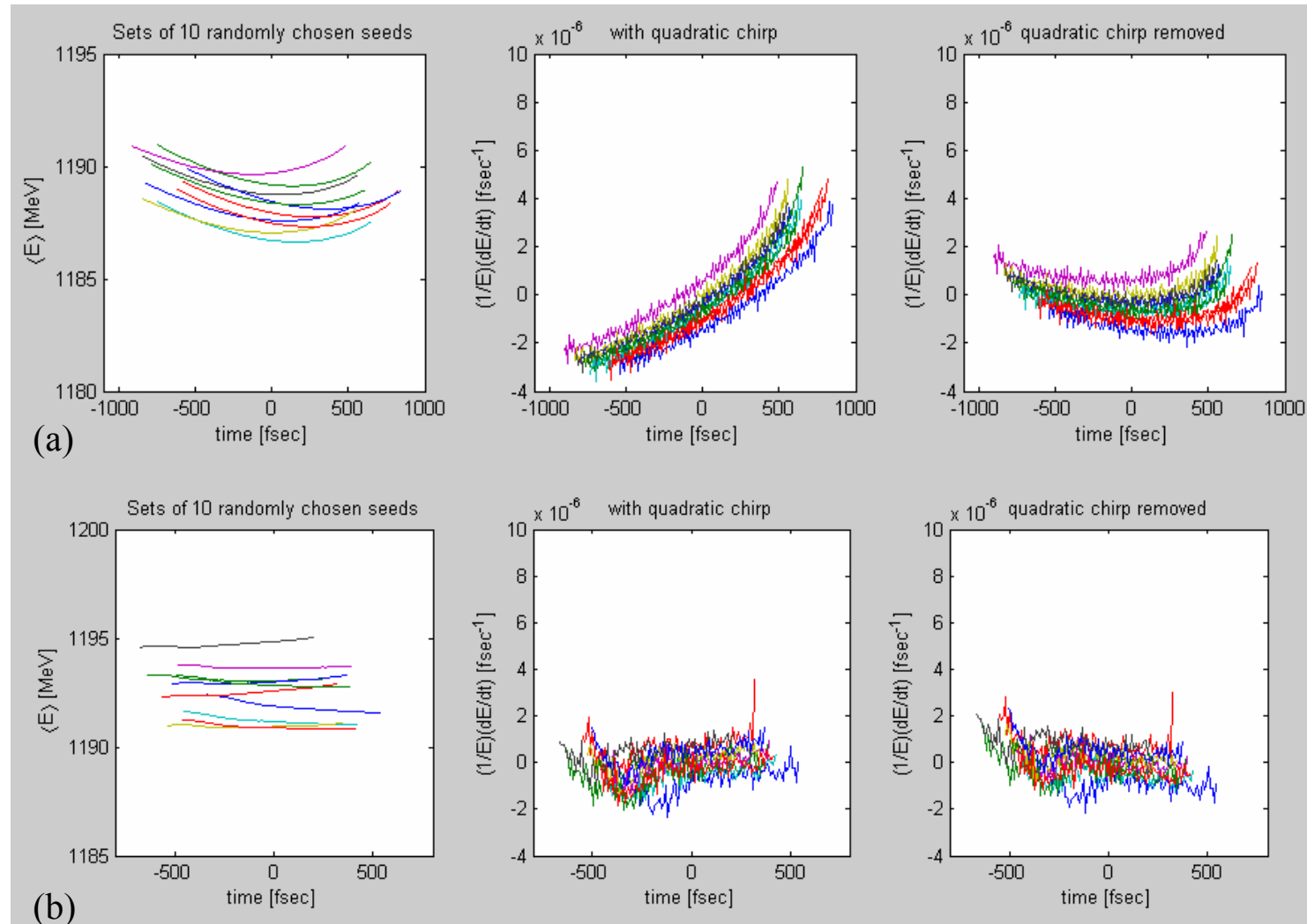

Figure (6.7) Set of 10 randomly chosen seeds for long bunch case L2 (a) and L4 (b) (left plots) together with relative first derivative of the energy with respect to time inside the bunch with quadratic chirp (central plots) and with quadratic chirp removed (right plots).

\subsection{Tracking studies with errors}

Consistent s2e simulations from the RF gun to the photon beam taking in account errors along different parts of the injector, the linac and the undulator were performed. Here are presented the preliminary results from Elegant tracking, with errors in the linac and in the photo-injector for medium case M2. Two codes were used: GPT [55] for photo-injector and Elegant for linac part. 84 different output particle distributions in the 6D phase space from GPT were randomly generated and converted to the Elegant input format. The particle distribution was tracked under the combinated influences of the linac errors with rms values taken from Table 6.5. As a result there are 84 output particle distributions at the linac end which were used as inputs in the FEL simulations. Statistical analysis on 84 different particle distributions obtained from Elegant are in good agreement with LiTrack results: $\Delta \mathrm{I}=\mathrm{I}<9.2 \% \mathrm{rms}, \Delta \mathrm{E}=\mathrm{E}<0.092 \%$ and $\Delta \mathrm{tf}<93 \mathrm{fs}$.It is interesting to notice that in GPT and Elegant runs charge and bunch compression variations were not accounted for. 

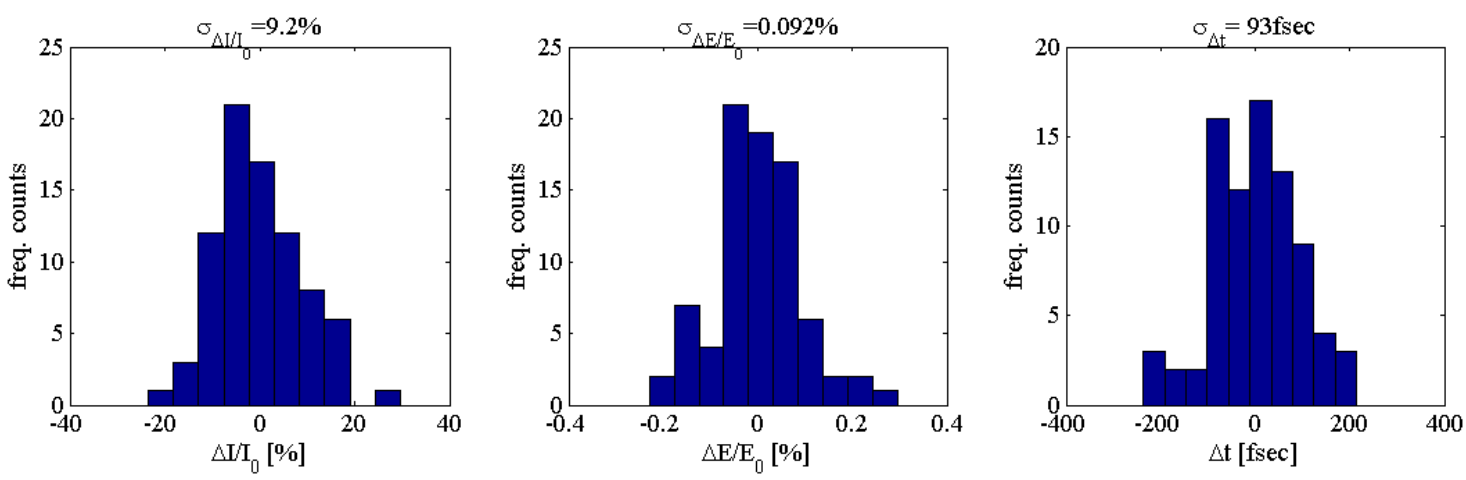

Figure (6.8) Histograms for medium bunch case M2 of the relative peak current $\Delta \mathrm{I} / \mathrm{I}_{0}$ (left), final relative mean energy $\Delta \mathrm{E} / \mathrm{E}_{0}$ (center) and final timing jitter $\Delta \mathrm{t}$ (right) obtained by means of Elegant simulations. $\mathrm{I}_{0}$ is the nominal peak current and $\mathrm{E}_{0}$ is the nominal mean energy.

\section{Appendix A: 6D parallel tracking code IMPACT}

IMPACT is a suite of parallel particle-in-cell codes, designed for modeling high intensity, high brightness beams in rf proton linacs, electron linacs and photoinjectors. It consists of two parallel particle-in-cell tracking codes (one is longitudinal position-dependent and one is time-dependent), an rf linac lattice design code, an envelope matching and analysis code, and a number of pre- and post-processing codes. The present version of IMPACT can treat intense beams propagating through drifts, magnetic quadrupoles, magnetic soleniods, magnetic dipole, and rf cavities, using map integrator and nonlinear Lorentz integrator. It has a novel treatment of rf cavities, in which the gap transfer maps are computed during the simulations by reading in rf fields on axis calculated using SUPERFISH code. The goal is to avoid time-consuming (and unnecessary) fine-scale integration of millions of particles through the highly z-dependent cavity fields. Instead, fine-scale integration is used to compute the maps (which involve a small number of terms), and the maps are applied to particles (this is analogous to the technique used to simulate beam transport through magnets with fringe fields). Recent additions include new capabilities for modeling short range longitudinal and transverse wakefields as well as 1D CSR wakes.

Both parallel particle tracking codes of the IMPACT suite assume a quasi-static model of the beam and calculate space-charge effect self-consistently at each time step together with the external acceleration and focusing fields. The 3D Poisson equation is solved by depositing the charge onto an adaptive 3D Cartesian grid and solving for the potential on the grid in the beam frame. The resulting electrostatic fields are Lorentz transformed back into the laboratory frame to obtain the space-charge forces of the beam. There are six Poisson solvers in the IMPACT code, corresponding to transverse open or closed boundary conditions with round or rectangular shape, and longitudinal open or periodic boundary conditions. These solvers use either a spectral method for closed transverse boundary conditions, or a convolution-based Green function method for open transverse boundary conditions. The convolution for the most widely used open boundary 
condition Poisson solver is calculated using an FFT with doubled computational domain. The computing time of this solver scales like $N x \log (N)$, where $N$ is number of grid points. The parallel implementation is based on a two-dimensional domain decomposition approach for the three-dimensional computational domain.

Typical IMPACT simulations at NERSC are run with a few millions to a few tens million particles, though smaller and larger runs are possible.

\section{Appendix B: Simulations using a Vlasov solver}

Direct numerical solution of the Vlasov equation represents a complementary approach to the more prevalent method of simulating beam dynamics by macroparticle traking. In particular a Vlasov solver offers the advantage of being immune from the statistical fluctuations stemming from using a limited number of macroparticles, which may interfere with a correct interpretation of the results when studying the microbunching instability. Small scale structures are more easily resolved and the unfolding of instabilities more accurately characterized. Moreover, in contrast to semi-analytical studies based on the linearized Vlasov equation, numerical solutions of the full equation can be used to investigate saturation effects, which may be important.

It should be pointed out, however, that the Vlasov solver should not be expected to substitute the macroparticle simulations as the burden both in terms of developing suitable numerical algorithms and usage of computational resources limits the dimensionality of phase space that can be explored. At this time only a 2D phase-space solver is available while significant more effort will have to be made for an upgrade to 4D. Moreover, the physics that can be currently investigated with the existing solver is limited in practice to short-scale effects of collective forces (microbunching).

Studies based on numerical solutions of the Vlasov equation should be seen as occupying a middle ground between the semi-analytical analysis based on the linearized Vlasov equation and macroparticle simulations.

Here we briefly discuss the application of the 2D longitudinal phase-space Vlasov solver that was recently developed and used to produce the data reported in Figure (2.17). The solver implemented as a Fortran code finds numerical solutions of the equation:

$$
\frac{\partial f}{\partial s}-\delta \frac{D}{R} \frac{\partial f}{\partial z}+F(z) \frac{\partial f}{\partial \delta}=0
$$

where

$$
F(z)=-\frac{e^{2} N c}{E_{0}} \int d k Z(k) \hat{\rho}(k) e^{i k z}
$$

is the collective force expressed in terms of an impedance per unit length $Z(k)$ and the Fourier transform of the longitudinal bunch density (with the sign in (B.2) dependent on the definition of $Z(k)$ ). The existing code allows to include models of impedance describing space charge, coherent synchrotron radiation (CSR), and RF structure wake fields. Space charge is modeled [56] in free space and in the approximation that the beam can be represented as a charge distribution with circular cross section and uniform transverse density. CSR [21] evaluated in free space on the assumption that the bunch follows a trajectory with uniform radius of curvature. This excludes transition effects 
through the entrance and exit of bending magnets. The model of RF structure wake fields uses the frequency domain formulation of the wake potentials reported in Sec. 2.2.2. However, In the study discussed here the RF structure wake fields were not included.

The numerical solution of (B.1) is found using a variation of the time-splitting method that was already used to study the longitudinal beam dynamics in storage rings [57]. A technical complication arising when treating single pass systems and in particular bunch compressors is the presence of a large correlation in the beam density in the $\mathrm{z}$ $\delta$ plane. The method implemented in the code goes around this complication by solving Eq. (B.1) in a new coordinate system where the correlation is removed. In the new coordinates the density function for the beam is represented on a rectangular grid with adapting cell sizes to follow the bunch compression in the longitudinal coordinate (and the corresponding stretching in canonical momentum). For a detailed description of the method we refer to [26].

A possible concern regarding the study of a purely longitudinal phase space is the seeming neglect of the smearing effect to microbunching caused by a finite transverse emittance. While it is true that a complete accurate assessment of the effect of transverse emittance should entail the inclusion of the dynamics in the horizontal plane as well, we argue that it is possible in $2 \mathrm{D}$ to account for this smearing in an approximate but meaningful way by introducing an effective low-pass filter in the evaluation of the collective force. In particular we contend that (B.2) should be replaced with

$$
F(z)=-\frac{e^{2} N c}{E_{0}} \int d k Z(k) \hat{\rho}(k) e^{i k z} \exp \left(-k^{2} \sigma_{\perp} / 2\right)
$$

where

$$
\sigma_{\perp}=\sqrt{2 \varepsilon_{x} H} ; \quad H=\gamma_{x} D^{2}+2 \alpha_{x} D D^{\prime}+\beta_{x} D^{\prime 2}
$$

For a derivation of (B.3) see [57]. Some confidence in the validity of the above model of emittance-induced smearing can be obtained from comparison with predictions from linear theory [22] in the regime where linear theory applies. Incidentally, such comparisons have also been successfully used to provide general validation of the coding. In Figure B1 we show the comparison between the gain curves as calculated from linear theory [22] (solid line) and the Vlasov solver (red dots) for BC1 in the presence of CSR only. The gain plotted is the ratio between the amplitude of a sinusoidal charge-density modulation for a given frequency at the exit and entry of the bunch compressor. The amplitude is understood to be expressed in units of the peak current (which is different at entrance and exit because of compression). The beam is a flattop with gaussian energy spread. We assumed a beam with vanishing transverse emittance, $10 \mathrm{KeV}$ rms energy spread, and $233 \mathrm{MeV}$ energy. As shown in the picture good agreement was found after an appropriate choice of the grid size and integration steps.

Figure B2 shows the gain curves for a beam that in addition travels through approximately $36 \mathrm{~m}$ of transport line (including linac 1) before entering the bunch compressor. Again, the gain curves are evaluated at the exit of $\mathrm{BC} 1$. In this case spacecharge effects are significant and are included in the calculation. The two figures contrast the case with vanishing and with $\varepsilon_{\mathrm{x}}=1 \mu \mathrm{m}$ normalized transverse emittance. Notice how the transverse emittance smearing effect substantially reduces the gain. Good agreement is found with linear theory. In the case with vanishing transverse emittance the Vlasov solver simulation is done without the low-pass filter in Eq. (B.3), however, a value 
$\varepsilon_{\mathrm{x}}=\varepsilon_{\mathrm{y}}=1 \mu \mathrm{m}$ is assumed for the purpose of determining the transverse sizes for spacecharge caclulations. The dependence of the space-charge force on the transverse size of the beam is treated slightly different in the two models. In the Vlasov code the local value of the transverse rms size (depending on the beta functions) is used so that the "effective" beam radius appearing in the impedance model is taken as $r_{b}^{2}=\left[1.3\left(\sigma_{x}^{2}+\sigma_{y}^{2}\right)\right]^{2}$. The 1.3 factor is an attempt to tune the model to reflect the fact that the form of the transverse charge density of the actual beam is closer to Gaussian than uniform. For the linear theory calculation a constant in $z$ transverse size is assumed. The parameter $r_{b}$ for the linear theory was adjusted to fit the gain curve against the Vlasov solver calculation. The same value, $r_{b}=0.27 \mathrm{~mm}$ was then used to produce both pictures in Figure B2.

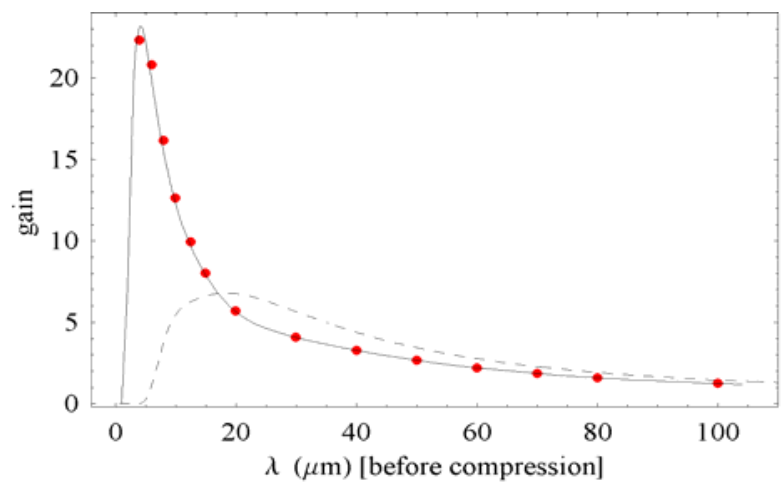

Figure B1. Gain curve for bunch-compressor BC1 in the presence of CSR (no space charge) as determined by linear theory (solid line) and from the numerical solutions of the Vlasov equation (red dot). Rms energy spread $\sigma_{\mathrm{E}}=10 \mathrm{KeV} ; \mathrm{E}=233 \mathrm{MeV}$; vanishing transverse emittance; peak current I=95 A (before compression); compression factor 3.52.
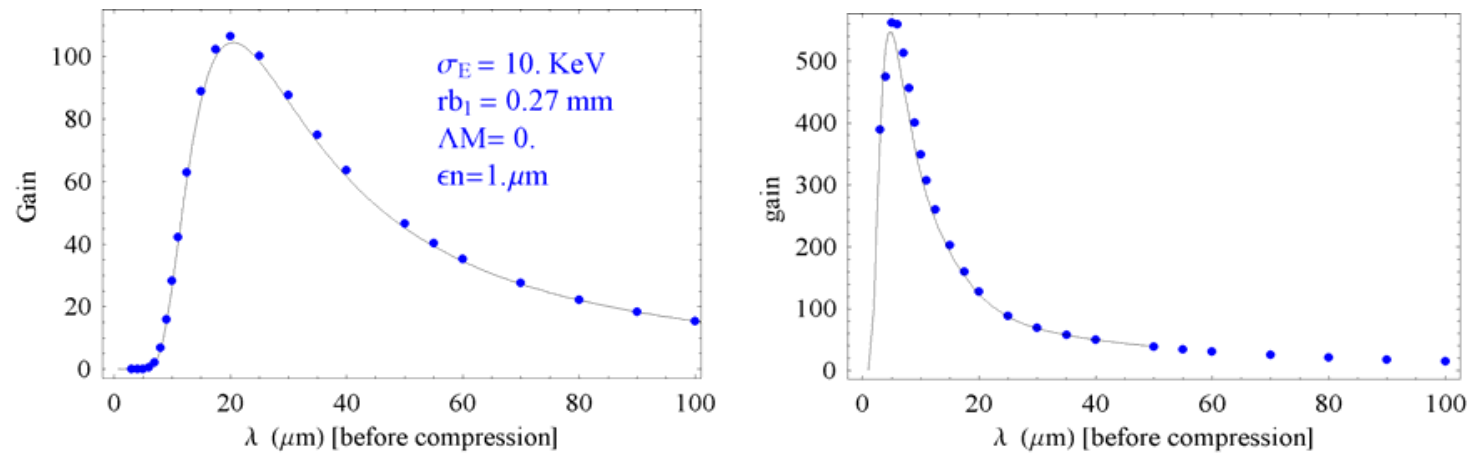

Figure B2. Gain curves through for $\mathrm{L} 1+\mathrm{BC} 1$ in the presence of $\mathrm{CSR}$ and space charge (with space charge excluded in $\mathrm{BC} 1$ ) as determined by linear theory (solid line) and from the numerical solutions of the Vlasov equation (blue dots). $\sigma_{\mathrm{E}}=10 \mathrm{KeV}$; smearing effect of transverse emittance is included in the left but not in the right picture. 
Having demonstrated consistency of results from the Vlasov solver with linear theory (at least over a portion of the FERMI accelerator) in the regime of small modulation amplitude where linear theory applies, we extended the calculation to include the linac 2 and linac 3 and the second bunch compressor BC2. The goal was to study the effect of shot noise in the development of the microbunching instability and determine its impact on the uncorrelated rms energy spread on the beam past $\mathrm{BC} 2$.

FERMI@Elettra through BC2
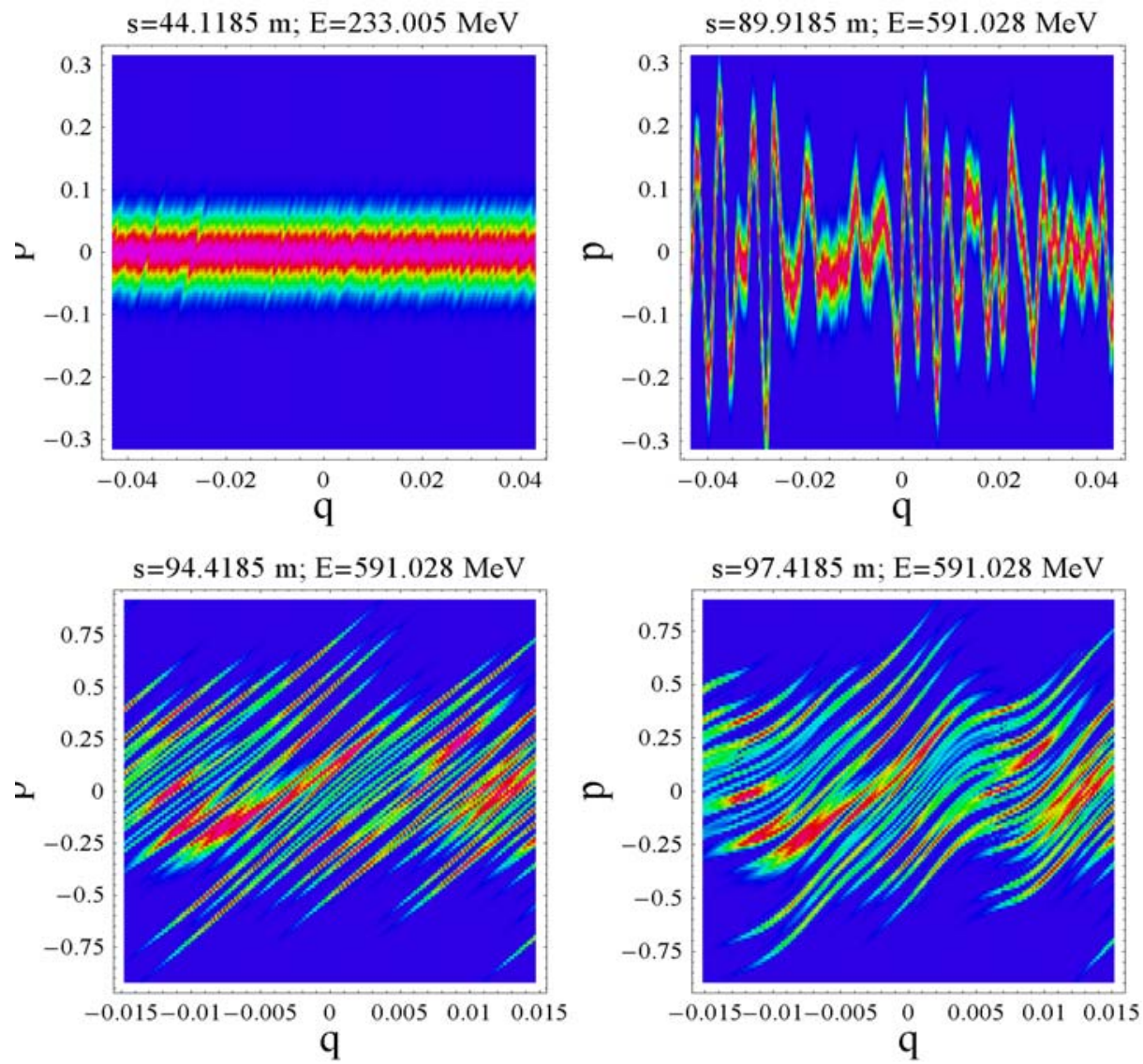

Figure B3. Longitudinal phase space at selected locations along the linacbunch/compressor complex starting from a noisy flat top bunch with gaussian rms energy spread; $s=0$ corresponds to the start of the laser-heater section. The top left pictures is taken at the exit of $\mathrm{BC} 1$; top right picture is taken at the entrance of $\mathrm{BC} 2$ and the remaining two after the $\mathrm{BC} 2$ third and fourth dipole. The coordinate $q$ is the longitudinal coordinate in units of $1 \mathrm{~mm}, p$ is the uncorrelated energy in $\mathrm{MeV}$.

The initial beam density is a flattop in charge density and Gaussian in the (uncorrelated) energy spread. The shot noise was modeled by perturbing the initial, smooth density function in phase space with random noise specified as follow. Having denoted with $(q, p)$ the pair of canonical coordinates ( $q$ is a normalized longitudinal coordinate, $p$ the energy) the density function $f=f\left(q_{i}, p_{j}\right)$ is represented on a Cartesian grid with cells of size $\Delta q$ and 
$\Delta p$. Indicate with $f_{i j}^{(0)}=f^{(0)}\left(q_{i}, p_{j}\right)$ he smooth density; we set the initial density with random noise to $f_{i j}=f_{i j}{ }^{(0)}\left(1+\xi_{i j}\right)$ where $\xi_{i j}$ is a stochastic variable with normal law distribution, vanishing average and variance $\sigma_{\xi}=N_{i j}{ }^{-1 / 2}$, where $N_{i j}=N f_{i j}^{(0)} \Delta q \Delta p$ is the number of electrons contained in the $\Delta q \Delta p$ cell of phase space. An example of the beam phase space at selected locations along the linac is reported in Figure B3 for a specific realization of the initial density with shot noise.

The calculation indicates that most of the energy modulation induced by space charge (the dominant collective effect) takes place between $\mathrm{BC} 1$ and $\mathrm{BC} 2$. By the time the beam reaches the second bunch compressor these fluctuations are sufficiently large to cause the instability to reach saturation, as indicated by the two bottom figures. We emphasize that in this calculation space charge and CSR are the only collective effects present. The overall bunch compression of 10 was distributed between bunch compression factors 3.52 and 2.84 in $\mathrm{BC} 1$ and $\mathrm{BC} 2$ respectively. These factors were obtained by suitable adjustment of the beam correlation at the start of linac 1 and by inserting an additional zero length cavity before $\mathrm{BC} 2$. The latter is necessary to compensate for the fact that in this model we lack the additional correlation caused by the RF structure wake fields.

Projection of the 2D phase-space density can then be made to determine the linear charge density profile and energy distribution and its variance. We repeated the calculation by varying the rms energy spread for the initial density in an attempt to model the effect of the laser heater. It should be pointed out however, that while we used a Gaussian distribution in (uncorrelated) energy spread the form of the beam distribution emerging from the interaction with the laser is somewhat different - possibly yielding different Landau damping for equal rms energy spreads. The results shown in Figure (2.17) clearly indicate an optimum range for the setting of the initial rms energy spread at about $\sigma_{E}=15 \mathrm{KeV}$. 


\section{References:}

[1] LCLS CDR, SLAC Report No. SLAC-R-593, 2002.

[2] TESLA TDR, DESY Report No. DESY-2001-011, 2001.

[3] J. S. Oh et al,. Proceedings of the 2004 FEL Conference, (2004)175.

[4] D. Moncton,W. Graves, The MIT X-ray Laser Project, Proc.of 2003 SRI Conference, p. 113, San Francisco, CA, August, (2003).

[5] BESSY FEL Technical Design Report, http://www.bessy.de, (2004).

[6] J.N.Corlett et al., Proceedings of 2004 European Particle Accelerator Conference, TPHKF075, Luzerne, (2004).

[7] 4GLS Conceptual Design Report, http://www.4gls.ac.uk/documents.htm, April (2006).

[8] D. H Dowell, T. D. .Hayward, A. M. Vetter, Proceedings of the 1995 Particle Accelerator Conference 1995, New York (1995).

[9] G. D'Auria, RF measurements on the 100MeV Linac Accelerating Sections, Ref. ST/M-TN-90/15,1990.

[10] K. Bane., Wakefields of sub-picosecond electron bunches, SLAC-PUB-11829, 2006.

[11] K. Bane, A. Mosnier, A. Novokhatski, K. Yokoya, in Proceedings of the International Computational Accelerator

Physics Conference, Monterey, CA, 1998 (SLAC, 1998) SLAC-R-580, p. 137.

[12] P. Craievich, T. Weiland, I. Zagorodnov, The short-range wakefields in the BTW accelerating structure of the

Elettra linac, ICAP 2004, St. Petersburg, URSS, June 2004.

[13] L.I. Schiff, Rev. Sci. Instr. 17 (1946)6;

J. S. Nodvick and D. S. Saxon, Phys. Rev. 96, 180 (1954);

R. L. Warnock and P. Morton, Part. Accel. 25, 113 (1990);

J.B. Murphy, S. Krinsky, and R.L. Gluckstern, PAC95, 2980 (1995); Part. Accl. 57, 9 (1997);

E.L. Saldin, E.A. Schneidmiller, and M.V. Yurkov, NIM A 398, (1997)373

[14] Ya.S. Derbenev, J. Rossbach, E.L. Saldin, and V.D. Shiltsev, DESY-TESLA-FEL-95-05 (1995).

[15] R.Li, C.L. Bohn, J.J. Bisongnano, Particle Accelerator Conference, (1997)1644.

[16] K.L.F. Bane P. Emma, LiTrack: A fast longitudinal phase space tracking code with graphical interface, PAC2005, Knoxville, Tennessee, (2005)4266.

[17] E.L. Saldin, E.A. Schneidmiller, and M.V. Yurkov, DESY Report No. TESLA-FEL- 2003-02, 2003.

[18] Z. Huang, M. Borland, P. Emma, J. Wu, C. Limborg, G. Stupakov, and J. Welch, Phys. Rev. ST Accel. Beams 7 , 074401 (2004).

[19] M. Borland, Y. Chae, P. Emma, J. Lewellen, V. Bharadwaj, W. Fawley, P. Krejcik, C. Limborg, S. Milton, H.-D. Nuhn, R. Soliday, and M. Woodley, Nucl. Instrum. Methods, Phys. Res. A 483, 268 (2002).

[20] E.L. Saldin, E.A. Schneidmiller, and M.V. Yurkov, Nucl. Instrum. Methods in Phys. Res. A 490, 1 (2002).

[21] S. Heifets, G. Stupakov, and S. Krinsky, Phys. Rev. ST Accel. Beams 5, (2002)064401.

[22] Z. Huang and K.-J. Kim, Phys. Rev. ST Accel. Beams 5, 074401 (2002).

[23] Z. Huang et al., PRST-AB, (2004)074401.

[24] M. Borland, "elegant"- A flexible SDDS-compliant code for accelerator simulations, APS Tech. Note LS-207, September 2000.

[25] J. Qiang IMPACT.

[26] M. Venturini, et al., LBNL Report, Berkeley, LBNL-60513 (2006)

[27] Ya.S. Derbenev, V.D. Shiltsev, FERMILAB-TM-1974, SLAC-Pub 7181 (1996).

[28] R.Li, Particle Accelerator Conference, New York, (1999)118.

[29] M. Dohlus, T. Limberg, P. Emma, Bunch compression for linac-based FELs, Beam dynamics newsletter, N38, Ed. I.S. Ko, (2005).

[30] P. Emma, Bunch Compressor Beamlines for the TESLA and S-band Linear Colliders, DESY Print, TESLA 95-17 (July 1995).

[31] T.O. Raubebenheimer, P. Emma, S. Kheifets, Chicane and Wiggler Based Bunch Compressors for Future Linear Colliders, Proc. of PAC 1993.

[32] M. Sands, Emittance Growth from Radiation Fluctuations, SLAC/AP-47 (December 1985)

[33] G. Leleux, P. Nghiem, A. Tkatchenko, Synchrotron radiation Perturbations in Long Transport Lines, Proc. of PAC 1991.

[34] H. Wiedemann, Particle Accelerator Physics Vol.I, pgg. 225 - 240, ed. Springer (second edition, 2003).

[35] S. Di Mitri et al., The Accelerator Design Study for the FERMI FEL, ST/F-TN-05/01 (March 2005)

[36] K. Bane et al., Calculation of the short-range longitudinal wakefields in the NLC Linac, SLACPUB-7862, 1998.

[37] K. Bane., Short-range dipole wakefields in accelerating structures for the NLC, SLAC-PUB-9663, 2003.

[38] J. S. Berg and R. D. Ruth, Transverse Multibunch Instabilities for Non-Rigid Bunches, IEEE (1996)

[39] R. D. Ruth et al., Manifold Damping of Transverse Wakefields in High Phase Advance Traveling Wave Structures and Local Damping of Dipole Wakefields in Standing Wave Accelerators, Proc. of the 2001 PAC, Chicago.

[40] V. Balakin, A. Novohatsky and V. Smirnov, in Proc. of the $12^{\text {th }}$ International Conf. on High Energy Accelerators, Batavia, Illinois (1983). Edited by F. T. Cole and R. Donaldson (Fermilab, Batavia, IL, 1984), p.119-120

[41] G. V. Stupakov, BNS Damping of Beam Breakup Instability, SLAC-AP-108 (August 1997)

[42] P. Tenenbaum, Improved Overhead Counting in the NLC Main Linac, SLAC-TN-04-047 (June 2004) 
[43] J. T. Seeman, Observation and Cures of Wakefield Effect in the SLC Linac, SLAC-PUB-5716 (December 1991)

[44] P. Emma, private communications

[45] P. Craievich and S. Di Mitri, Emittance Growth due to Short-Range Transverse Wakefields in the Fermi Linac, Proc. of the FEL 2005 Conf., SLAC, CA (June 2005)

[46] S. Di Mitri et al., Optimization and Modeling of the Accelerator for the FERMI @, Elettra FEL, Proc. of the FEL 2005 Conf., SLAC, CA (June 2005)

[47] A.W. Chao, B. Richter and C.Y. Yao, Nucl. Instrum. Methods 178, 1 (1980).

[48] J.R. Delayen, Phys. Rev. ST Accel. Beams, 6, 084402 (2003).

[49] J.R. Delayen, Phys. Rev. ST Accel. Beams, 7, 074402 (2004).

[50] G. De Ninno, B. Fawley and G. Penn, Effect of Off-Axis Displacement Errors on FEL-1, ST/F-TN-06/01 (CBP Tech Note-356).

$[51]\left|\Delta \mathrm{b}_{\mathrm{k}} / \mathrm{b}_{\mathrm{n}, 0}\right|=\left\{\left[\Delta \int_{\mathrm{L}}\left(\partial^{\mathrm{k}} \mathrm{B}_{\mathrm{y}} / \partial \mathrm{x}^{\mathrm{k}}\right) \mathrm{d} l\right] \cdot\left[\int_{\mathrm{L}}\left(\partial^{\mathrm{n}} \mathrm{B}_{\mathrm{y}} / \partial \mathrm{x}^{\mathrm{n}}\right) \mathrm{d} l\right]^{-1}\right\} \cdot\left(\mathrm{R}^{\mathrm{k}} / \mathrm{R}^{\mathrm{n}}\right)$ with $\mathrm{n}=0,1,2$, etc $\ldots$

[52] M. Budiman, Matlab utility: Latin Hypercube Sampling, budiman@acss.usyd.edu.au, 2004.

[53] R. L. Iman and W. J. Conover, A distribution-free approach to inducing rank correlation among input variables, in Communications in Statistics B11, pp. 311-334, 1982.

[54] G. D. Wyss and K. H. Jorgensen, A Users Guide to LHS: Sandias Latin Hypercube Sampling Software, Technical Report SAND98-0210, Sandia National Laboratories, Albuquerque, NM, 1998.

[55] S. B. van der Geer et al., http://www.pulsar.nl/gpt.

[56] J. Rosenzweig, et al., NIM-A, 393 (1997) 376-379.

[57] M. Venturini, et al., PRST-AB 8, 014202 (2005). 